\title{
MATERIALS FOR THE STUDY OF DEEP-FOCUS EARTHQUAKES*
}

\author{
By B. Gutenberg and C. F. Richter
}

\section{INTRODUCTION}

IN A SERIES of recent papers ${ }^{1}$ the authors have presented a body of data and conclusions dealing with seismic waves in normal earthquakes. In the present paper these will be referred to as "On Seismic Waves, I, II, III." Before one proceeds to the investigation of deep-focus shocks, certain fundamental data must be derived from the results found for normal earthquakes. This is the purpose of the present paper. The theoretical results can then be compared with the observations of deep-focus earthquakes.

Certain preliminary conclusions for deep-focus shocks have been given in a previous paper ${ }^{2}$ the same material is here revised and extended.

\section{Methods for Calculating Travel Times of Deep-Focus Earthouakes}

Any calculation of travel times for a shock occurring at given depth requires knowledge of the distribution of wave velocity with depth, such as is given in "On Seismic Waves, II."

The straightforward procedure involves an integration along each path from source to surface; this is laborious, and accordingly we have made use of methods which reduce the amount of direct computation required. If the travel-time curve is known for waves originating at depth $A$, then the corresponding times for a depth $B$, greater than $A$, can be found by subtracting $\int_{A}^{B} \frac{\tan i}{r} d r$ from the epicentral distance $\Delta$, and subtracting $\int_{A}^{B} \frac{d r}{v \cos i}$ from the corresponding travel time ( $i=$ angle of incidence, $r=$ radius, $v=$ velocity). The values of these corrections depend only on the nature (longitudinal or transverse) of the wave as it leaves the source, and on its initial direction (initial value of the angle $i$ ). Thus there will be only two tabulations of corrections, one for $\mathrm{P}$ and one for $\mathrm{S}$. Those derived for $\mathrm{P}$ will apply directly to $\mathrm{pP}, \mathrm{PP}, \mathrm{pS}, \mathrm{PS}, \mathrm{PKS}$, and so forth; those for $\mathrm{S}$ will apply directly to S, sS, SS, sP, SP, SKP, SKS, and so forth.

This method permits of deriving the travel times of deep-focus earthquakes from the normal travel times, wherever tabulations for the phases being studied have been constructed for normal shocks. Since such tabulations are not avail-

* Manuscript received for publication August 16, 1936.

1 B. Gutenberg and C. F. Richter, "On Seismic Waves," Gerlands Beitr. z. Geophys., 43:56-133 (1934); second paper, ibid., 45:280-360 (1935); third paper, ibid., 47:73-131 (1936).

${ }^{2}$ B. Gutenberg and C. F. Richter, "Contribution to the Study of Deep-Focus Earthquakes," Gerlands Beitr. z. Geophys., 41:160-169 (1934). 
able for phases such as $\mathrm{pP}, \mathrm{sP}, \mathrm{pS}$, either they must be constructed for the present purpose or a slightly different procedure must be adopted.

In practice, the times of $\mathrm{pP}$ are derived from those of $\mathrm{P}$. The corrections in time and distance, which are to be added, each consist of the sum of two integrals from 0 to $A$ and 0 to $B$, the integrands having the same form as given above. In dealing with "mixed" waves such as pS or sP, the integral from 0 to $A$ must be taken for the type of wave after the first reflection, and that from 0 to $B$ for the type of wave leaving the focus.

For waves which are purely longitudinal or purely transverse, the corrections in time and distance to be applied to the normal travel times (for an assumed depth of $25 \mathrm{~km}$.) are given in tables 1 to 4 inclusive, for 0 to $800 \mathrm{~km}$. focal depth

TABLE 1

Distance Corrections, in Degrees, for Longitudinal Waves

\begin{tabular}{|c|c|c|c|c|c|c|c|c|c|}
\hline \multirow{2}{*}{ 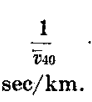 } & \multicolumn{9}{|c|}{ Depth of focus in $\mathrm{km}$. } \\
\hline & 0 & 100 & 200 & 300 & 400 & 500 & 600 & 700 & 800 \\
\hline
\end{tabular}

(a) For waves starting downward (symbol beginning with $\mathrm{P}$ )

\begin{tabular}{l|l|l|l|l|l|l|l|l|l}
\hline 0.01 & 0.0 & -0.1 & -0.1 & -0.2 & -0.3 & -0.4 & -0.5 & -0.6 & -0.7 \\
0.02 & 0.0 & -0.1 & -0.3 & -0.4 & -0.6 & -0.8 & -1.0 & -1.3 & -1.5 \\
0.03 & 0.0 & -0.2 & -0.4 & -0.7 & -0.9 & -1.2 & -1.6 & -2.0 & -2.4 \\
0.04 & 0.1 & -0.2 & -0.5 & -0.9 & -1.2 & -1.7 & -2.2 & -2.7 & -3.3 \\
0.05 & 0.1 & -0.3 & -0.7 & -1.1 & -1.6 & -2.2 & -2.9 & -3.6 & -4.4 \\
& & & & & & & & & \\
0.06 & 0.1 & -0.4 & -0.9 & -1.4 & -2.1 & -2.8 & -3.7 & -4.7 & -5.8 \\
0.07 & 0.1 & -0.5 & -1.1 & -1.8 & -2.6 & -3.6 & -4.8 & -6.1 & -7.9 \\
0.08 & 0.1 & -0.6 & -1.3 & -2.2 & -3.3 & -4.7 & -6.6 & & \\
0.09 & 0.1 & -0.7 & -1.5 & -2.9 & -4.6 & -6.6 & & & \\
0.10 & 0.2 & -0.9 & -2.2 & -3.7 & -6.8 & & & & \\
\hline
\end{tabular}

(b) For waves starting upward (symbol beginning with $\mathrm{p}$ )

\begin{tabular}{l|l|l|l|l|l|l|l|l|l}
\hline 0.01 & 0.1 & 0.1 & 0.2 & 0.3 & 0.4 & 0.5 & 0.6 & 0.7 \\
0.02 & 0.2 & 0.3 & 0.4 & 0.6 & 0.8 & 1.0 & 1.2 & 1.5 \\
0.03 & 0.2 & 0.5 & 0.7 & 1.0 & 1.3 & 1.7 & 2.0 & 2.4 \\
0.04 & & 0.3 & 0.7 & 1.0 & 1.4 & 1.8 & 2.3 & 2.8 & 3.4 \\
0.05 & 0.4 & 0.9 & 1.3 & 1.8 & 2.4 & 3.0 & 3.7 & 4.5 \\
0.06 & & & & & & & & \\
0.07 & & & & & & & & & \\
0.08 & 0.5 & 1.1 & 1.6 & 2.2 & 3.0 & 3.9 & 4.8 & 5.9 \\
0.09 & 0.8 & 1.3 & 2.0 & 2.8 & 3.7 & 4.9 & 6.3 & 8.2 \\
0.10 & 1.5 & 2.4 & 3.5 & 4.9 & 6.7 & & \\
\hline
\end{tabular}


at intervals of $100 \mathrm{~km}$. The parts of tables lettered $(a)$ give the values of the integrals from $A$ to $B(A=25 \mathrm{~km} ., B$ variable), and those lettered $(b)$ give the values of the sum of the integrals from 0 to $A$ and 0 to $B$, assuming the same type of wave in both integrations. Corrections for "mixed" waves can be derived by an appropriate combination of the tables. These tables have been calculated from the results in "On Seismic Waves, II."

Instead of the angle of incidence, the corrections in these tables are given as functions of the quantity $\frac{1}{\bar{v}_{40}}$, the reciprocal of the apparent velocity of the wave at the base of the crust, which is taken at a depth of $40 \mathrm{~km}$. The values of this quantity have been calculated and used in "On Seismic Waves, II."

TABLE 2

Travel-Time Corrections, in Seconds, for Longitudinal Waves

\begin{tabular}{c|c|c|c|c|c|c|c|c|c|c|}
\hline $\begin{array}{c}1 \\
\vec{v}_{40} \\
\text { sec/km. }\end{array}$ & \multicolumn{10}{c|}{ Depth of focus in $\mathrm{km}}$. \\
\hline
\end{tabular}

(a) For waves starting downward

\begin{tabular}{l|l|l|l|l|l|l|l|l|l}
\hline 0.00 & 4 & -10 & -23 & -35 & -46 & -57 & -67 & -76 & -85 \\
0.02 & 4 & -10 & -23 & -35 & -46 & -57 & -67 & -77 & -86 \\
0.03 & 4 & -10 & -24 & -36 & -47 & -58 & -68 & -79 & -89 \\
0.04 & 4 & -10 & -24 & -36 & -48 & -60 & -71 & -82 & -92 \\
0.05 & 5 & -11 & -25 & -37 & -50 & -63 & -74 & -86 & -97 \\
0.06 & 5 & -11 & -26 & -39 & -53 & -66 & -79 & -92 & -106 \\
0.07 & 5 & -12 & -28 & -42 & -57 & -71 & -87 & -104 & -123 \\
0.08 & 5 & -13 & -30 & -45 & -63 & -81 & -102 & & \\
0.09 & 5 & -14 & -32 & -51 & -75 & -98 & & & \\
0.10 & 6 & -15 & -35 & -61 & -98 & & & & \\
\hline
\end{tabular}

(b) For waves starting upward

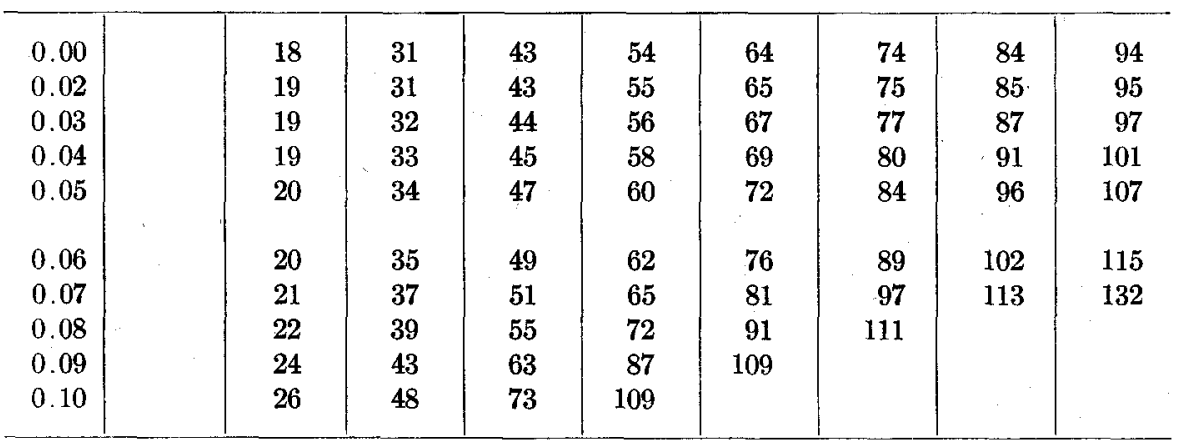


TABLE 3

Distance Corrections, in Degrees, for Transverse Waves

\begin{tabular}{c|c|c|c|c|c|c|c|c|c}
\hline $\begin{array}{c}\bar{v}_{40} \\
\text { sec/km. }\end{array}$ & \multicolumn{10}{c}{ Depth of focus in $\mathrm{km}}$. \\
\hline
\end{tabular}

(a) For waves starting downward (symbol beginning with $\mathbf{S}$ )

\begin{tabular}{l|l|l|l|l|l|l|l|l|l}
\hline 0.02 & 0.0 & -0.0 & -0.1 & -0.2 & -0.3 & -0.5 & -0.6 & -0.7 & -0.8 \\
0.04 & 0.1 & -0.1 & -0.3 & -0.5 & -0.7 & -0.9 & -1.2 & -1.4 & -1.7 \\
0.06 & 0.1 & -0.1 & -0.4 & -0.7 & -1.0 & -1.3 & -1.7 & -2.1 & -2.6 \\
0.07 & 0.1 & -0.2 & -0.5 & -0.8 & -1.2 & -1.6 & -2.0 & -2.6 & -3.2 \\
0.08 & 0.1 & -0.2 & -0.6 & -1.0 & -1.4 & -1.9 & -2.4 & -3.0 & -3.7 \\
0.09 & 0.1 & -0.3 & -0.7 & -1.2 & -1.6 & -2.2 & -2.8 & -3.5 & -4.3 \\
& & & & & & & & & \\
0.10 & 0.1 & -0.3 & -0.8 & -1.3 & -1.9 & -2.5 & -3.3 & -4.1 & -5.0 \\
0.11 & 0.1 & -0.4 & -0.9 & -1.5 & -2.2 & -2.9 & -3.7 & -4.8 & -5.8 \\
0.12 & 0.1 & -0.4 & -1.0 & -1.7 & -2.5 & -3.3 & -4.3 & -5.6 & -6.7 \\
0.13 & 0.1 & -0.5 & -1.1 & -1.9 & -2.8 & -3.8 & -4.9 & -6.4 & -8.3 \\
0.14 & 0.2 & -0.6 & -1.3 & -2.2 & -3.2 & -4.5 & -6.2 & & \\
0.15 & 0.2 & -0.7 & -1.5 & -2.6 & -4.0 & -5.8 & & & \\
0.16 & 0.2 & -0.7 & -1.8 & -3.2 & -5.0 & -7.5 & & & \\
0.17 & 0.2 & -0.8 & -2.2 & -3.8 & & & & & \\
0.18 & 0.2 & -0.9 & -2.5 & -4.6 & & & & & \\
0.19 & 0.2 & -1.1 & -2.9 & -5.5 & & & & \\
\hline
\end{tabular}

(b) For waves starting upward (symbol beginning with s)

\begin{tabular}{l|l|l|l|l|l|l|l|l|l}
\hline 0.02 & & 0.1 & 0.2 & 0.3 & 0.4 & 0.5 & 0.6 & 0.7 & 0.9 \\
0.04 & 0.2 & 0.3 & 0.5 & 0.7 & 0.9 & 1.2 & 1.4 & 1.7 \\
0.06 & 0.2 & 0.5 & 0.8 & 1.1 & 1.4 & 1.8 & 2.3 & 2.8 \\
0.07 & 0.3 & 0.6 & 1.0 & 1.4 & 1.7 & 2.2 & 2.8 & 3.3 \\
0.09 & & 0.3 & 0.7 & 1.1 & 1.6 & 2.0 & 2.6 & 3.2 & 3.9 \\
0.10 & 0.4 & 0.8 & 1.3 & 1.8 & 2.3 & 2.9 & 3.7 & 4.5 \\
0.11 & & & & & & & & \\
0.12 & 0.5 & 0.9 & 1.5 & 2.0 & 2.6 & 3.4 & 4.3 & 5.2 \\
0.13 & 0.6 & 1.0 & 1.7 & 2.3 & 3.0 & 3.9 & 5.0 & 6.0 \\
0.14 & 0.7 & 1.2 & 1.9 & 2.6 & 3.5 & 4.5 & 5.8 & 7.0 \\
0.15 & 0.8 & 1.4 & 2.2 & 3.0 & 4.0 & 5.3 & 6.7 & 8.6 \\
0.16 & 0.9 & 1.6 & 2.5 & 3.5 & 4.8 & 6.5 & & \\
0.17 & & & & & & & & & \\
0.18 & 1.0 & 1.9 & 3.0 & 4.3 & 6.1 & & & \\
0.19 & 1.1 & 2.2 & 3.5 & 5.3 & 7.8 & & & \\
\hline
\end{tabular}


TABLE 4

Travel-'Time Corrections, in Seconds, for Transverse Waves

\begin{tabular}{|c|c|c|c|c|c|c|c|c|c|}
\hline \multirow{2}{*}{$\begin{array}{c}\frac{1}{\bar{v} 4_{0}} \\
\mathrm{sec} / \mathrm{km}\end{array}$} & \multicolumn{9}{|c|}{ Depth of foeus in $\mathrm{km}$. } \\
\hline & 0 & 100 & 200 & 300 & 400 & 500 & 600 & 700 & 800 \\
\hline
\end{tabular}

(a) For waves starting downward

\begin{tabular}{l|l|l|l|l|l|l|l|l|l}
\hline 0.00 & 7 & -17 & -40 & -61 & -82 & -101 & -120 & -137 & -154 \\
0.04 & 7 & -17 & -40 & -62 & -84 & -104 & -123 & -140 & -157 \\
0.06 & 7 & -17 & -41 & -64 & -86 & -107 & -127 & -146 & -164 \\
0.08 & 8 & -18 & -42 & -66 & -88 & -111 & -132 & -152 & -172 \\
0.09 & 8 & -18 & -43 & -67 & -90 & -113 & -135 & -156 & -177 \\
& & & & & & & & & \\
0.10 & 8 & -19 & -44 & -69 & -93 & -116 & -138 & -162 & -184 \\
0.11 & 8 & -19 & -45 & -71 & -96 & -119 & -144 & -168 & -194 \\
0.12 & 8 & -20 & -47 & -73 & -99 & -125 & -151 & -177 & -207 \\
0.13 & 9 & -21 & -49 & -76 & -102 & -132 & -159 & -188 & -224 \\
0.14 & 9 & -22 & -51 & -80 & -109 & -141 & -172 & & \\
0.15 & 9 & -23 & -54 & -84 & -117 & -152 & & & \\
0.16 & 9 & -24 & -57 & -90 & -126 & -169 & & & \\
0.17 & 9 & -26 & -62 & -97 & & & & & \\
0.18 & 10 & -28 & -67 & -109 & & & & & \\
0.19 & 10 & -31 & -76 & -135 & & & & & \\
\hline
\end{tabular}

(b) For waves starting upward

\begin{tabular}{|c|c|c|c|c|c|c|c|c|}
\hline 0.00 & 31 & 54 & 75 & 96 & 115 & 134 & 151 & 168 \\
\hline 0.04 & 31 & 55 & 77 & 99 & 118 & 137 & 155 & 172 \\
\hline 0.06 & 32 & 56 & 79 & 100 & 121 & 142 & 160 & 178 \\
\hline 0.08 & 33 & 58 & 81 & 102 & 125 & 146 & 167 & 187 \\
\hline 0.09 & 34 & 59 & 83 & 105 & 127 & 150 & 171 & 192 \\
\hline 0.10 & 35 & 60 & 85 & 108 & 131 & 155 & 177 & 199 \\
\hline 0.11 & 36 & 61 & 87 & 111 & 136 & 161 & 184 & 210 \\
\hline 0.12 & 37 & 63 & 90 & 115 & 142 & 168 & 193 & 223 \\
\hline 0.13 & 38 & 65 & 93 & 120 & 149 & 176 & 205 & 241 \\
\hline 0.14 & 39 & 68 & 96 & 126 & 158 & 189 & & \\
\hline 0.15 & 40 & 71 & 101 & 134 & 170 & & & \\
\hline 0.16 & 42 & 75 & 108 & 144 & 186 & & & \\
\hline 0.17 & 44 & 80 & 118 & & & & & \\
\hline 0.18 & 47 & 86 & 131 & & & & & \\
\hline 0.19 & 50 & 95 & 145 & & & & & \\
\hline
\end{tabular}


In finding travel times from the tabulated corrections, due attention has to be paid to the phenomenon of minimum distances for reflected phases (see the following section).

A valuable and sensitive check on the travel times, when they are available as functions of distance and focal depth, is the relation

$$
\left(\frac{\partial t}{\partial r}\right)^{2}+\frac{1}{r^{2}}\left(\frac{\partial t}{\partial \theta}\right)^{2}=\frac{1}{v^{2}}
$$

in which $r, \theta$ are polar coördinates, and $v$ is the velocity at the depth considered. In the first term $\frac{\partial t}{\partial r}$ may be replaced by $\frac{\partial t}{\partial h}$, where $h$ is the depth. The use of this relation is facilitated by computing from the known velocity distribution a table of corresponding values of $\frac{\partial t}{\partial \theta}$ and $\frac{\partial t}{\partial h}$ (table 5).

\section{TABLE 5}

\section{Corresponding Values of $d t / d \theta$ and $d t / d h$}

( $d t / d \theta$ gives the change in travel time corresponding to a change in distance of one degree at a given point of a travel-time curve, $d t / d h$ the change in travel time at the same point, if the distance is kept unchanged, but the depth of focus is changed; $d t / d h$ is given in sec/100 $\mathrm{km}$. )

\begin{tabular}{c|c|c|c|c|c|c|c|c|c}
\hline $\begin{array}{c}\frac{7}{d \theta} \\
\sec / 1^{\circ}\end{array}$ & \multicolumn{10}{c}{ Depth of focus in km. } \\
\hline
\end{tabular}

(a) $\frac{d t}{d h}$ for waves which leave the focus as longitudinal waves

\begin{tabular}{r|r|r|r|r|r|r|r|r|r}
\hline 13 & 3.8 & 2.7 & & & & & & & \\
12 & 6.0 & 5.3 & 3.5 & & & & & & \\
11 & 7.4 & 6.9 & 5.5 & 2.0 & & & & & \\
10 & 8.5 & 8.2 & 7.0 & 4.9 & 2.0 & & & & \\
9 & 9.4 & 9.1 & 8.0 & 6.4 & 4.8 & 3.4 & 2.0 & & \\
8 & 10.2 & 9.9 & 8.8 & 7.5 & 6.3 & 5.4 & 4.7 & 3.6 & 2.0 \\
7 & 10.7 & 10.5 & 9.6 & 8.3 & 7.2 & 6.5 & 6.0 & 5.4 & 4.6 \\
6 & 11.2 & 11.0 & 10.3 & 9.0 & 8.1 & 7.5 & 7.0 & 6.5 & 6.0 \\
5 & 11.7 & 11.5 & 10.8 & 9.6 & 8.7 & 8.2 & 7.8 & 7.3 & 6.9 \\
4 & 12.1 & 11.8 & 11.2 & 10.1 & 9.2 & 8.7 & 8.4 & 8.0 & 7.7 \\
3 & 12.3 & 12.0 & 11.4 & 10.4 & 9.5 & 9.1 & 8.8 & 8.5 & 8.2 \\
2 & 12.4 & 12.2 & 11.6 & 10.6 & 9.8 & 9.3 & 9.1 & 8.8 & 8.5 \\
0 & 12.5 & 12.3 & 11.7 & 10.8 & 10.0 & 9.5 & 9.2 & 9.0 & 8.8 \\
\hline
\end{tabular}


TABLE 5-(Concluded)

\begin{tabular}{c|c|c|c|c|c|c|c|c|c}
\hline $\begin{array}{c}\frac{d t}{d \theta} \\
\text { sec } / 1^{\circ}\end{array}$ & \multicolumn{10}{c|}{ Depth of focus in $\mathrm{km}}$. \\
\hline
\end{tabular}

(b) $\frac{d t}{d h}$ for waves which leave the focus as transverse waves

\begin{tabular}{r|r|r|r|r|r|r|r|r|r}
\hline 24 & 5.0 & & & & & & & & \\
23 & 8.0 & 6.6 & & & & & & & \\
22 & 10.0 & 8.9 & 2.0 & & & & & & \\
21 & 11.5 & 10.7 & 6.5 & & & & & & \\
20 & 12.8 & 12.0 & 8.9 & 5.0 & & & & & \\
19 & 14.1 & 13.4 & 10.7 & 7.5 & 1.8 & & & & \\
18 & 15.2 & 14.6 & 12.2 & 9.6 & 6.2 & & & & \\
17 & 16.2 & 15.6 & 13.3 & 11.2 & 8.9 & 5.6 & & & \\
16 & 17.1 & 16.5 & 14.3 & 12.3 & 10.5 & 8.2 & 5.2 & & \\
15 & 17.8 & 17.3 & 15.3 & 13.5 & 11.8 & 9.8 & 7.6 & 5.2 & \\
14 & 18.5 & 18.0 & 16.2 & 14.4 & 12.9 & 11.2 & 9.5 & 7.5 & 3.9 \\
13 & 19.1 & 18.7 & 17.0 & 15.3 & 13.9 & 12.3 & 10.9 & 9.3 & 7.0 \\
12 & 19.6 & 19.2 & 17.6 & 16.1 & 14.7 & 13.2 & 11.9 & 10.5 & 8.7 \\
11 & 20.1 & 19.7 & 18.1 & 16.8 & 15.4 & 14.1 & 12.9 & 11.6 & 10.0 \\
10 & 20.5 & 20.2 & 18.6 & 17.4 & 16.0 & 14.8 & 13.7 & 12.6 & 11.1 \\
8 & 21.2 & 20.9 & 19.4 & 18.3 & 17.0 & 16.0 & 15.0 & 14.1 & 12.9 \\
6 & 21.8 & 21.5 & 20.1 & 18.9 & 17.8 & 16.7 & 15.9 & 15.1 & 14.2 \\
4 & 22.1 & 21.9 & 20.5 & 19.4 & 18.3 & 17.4 & 16.5 & 15.8 & 15.0 \\
2 & 22.3 & 22.1 & 20.8 & 19.7 & 18.6 & 17.7 & 16.9 & 16.1 & 15.3 \\
0 & 22.4 & 22.2 & 20.9 & 19.8 & 18.7 & 17.8 & 17.0 & 16.2 & 15.4 \\
\hline
\end{tabular}

\section{Calculated Travel Times}

By use of the methods given in the preceding section, travel times have been calculated for all the principal seismic waves, for focal depths of 100 to $800 \mathrm{~km}$. at intervals of $100 \mathrm{~km}$. These are given as tables 6 to 44 inclusive.

In dealing with waves which show multiplicity in normal shocks, the earliest wave has been used where it is defined by a clear travel-time curve (as for P). In dealing with other waves the travel times of the strongest wave of each group have been used.

In table 7 the times for $\mathrm{P}^{\prime}$ at the shorter distances (less than the focal distance $\Delta^{\prime}$ tabulated in the last lines of the table) refer to the "diffracted" $\mathrm{P}$ ". These times have been calculated by the same procedure as that used for the direct $\mathrm{P}^{\prime}$. There is some question of how far this method is applicable, since the two parts of the ray in the mantle may not be symmetrical. Consequently, the times calculated for the "diffracted" P' may be appreciably in error. 
TABLE 6

Travel Trmes of P in Min.: Sec.

\begin{tabular}{|c|c|c|c|c|c|c|c|c|}
\hline \multirow{2}{*}{$\begin{array}{l}\text { Distance } \\
\text { in deg. }\end{array}$} & \multicolumn{8}{|c|}{ Depth of focus in $\mathrm{km}$. } \\
\hline & 100 & 200 & 300 & 400 & 500 & 600 & 700 & 800 \\
\hline 0. & $0: 14$ & $0: 27$ & $0: 39$ & $0: 50$ & 1:01 & $1: 11$ & $1: 20$ & $1: 29$ \\
\hline 2. & $0: 30$ & $0: 38$ & $0: 46$ & $0: 57$ & 1:06 & $1: 15$ & $1: 24$ & $1: 32$ \\
\hline $4 .$. & $0: 56$ & $1: 03$ & $1: 08$ & $1: 12$ & $1: 18$ & $1: 25$ & $1: 32$ & $1: 40$ \\
\hline $6 .$. & $1: 25$ & $1: 28$ & $1: 30$ & $1: 30$ & $1: 34$ & $1: 40$ & $1: 46$ & $1: 52$ \\
\hline 8.. & $1: 54$ & $1: 54$ & $1: 54$ & $1: 53$ & $1: 54$ & 1:59 & $2: 03$ & $2: 07$ \\
\hline 10. & $2: 22$ & $2: 20$ & $2: 18$ & $2: 16$ & $2: 16$ & $2: 18$ & $2: 20$ & $2: 24$ \\
\hline 12. & $2: 50$ & $2: 46$ & $2: 42$ & $2: 39$ & $2: 38$ & $2: 38$ & $2: 38$ & 2:41 \\
\hline 14. & $3: 17$ & $3: 12$ & 3:07 & $3: 02$ & $3: 00$ & $2: 59$ & $2: 57$ & $2: 59$ \\
\hline 16. & $3: 43$ & $3: 37$ & $3: 31$ & $3: 25$ & $3: 22$ & $3: 19$ & $3: 17$ & $3: 17$ \\
\hline 18. & 4:07 & $4: 00$ & $3: 54$ & $3: 47$ & $3: 43$ & $3: 39$ & $3: 36$ & $3: 35$ \\
\hline $20 \ldots$ & $4: 30$ & $4: 22$ & $4: 15$ & $4: 08$ & 4:03 & $3: 58$ & $3: 55$ & $3: 53$ \\
\hline 22 & $4: 51$ & $4: 43$ & $4: 34$ & $4: 27$ & $4: 22$ & 4:16 & $4: 12$ & $4: 10$ \\
\hline 24. & $5: 10$ & $5: 02$ & $4: 53$ & 4:45 & $4: 39$ & $4: 34$ & 4:30 & $4: 27$ \\
\hline 26. & $5: 29$ & $5: 20$ & $5: 11$ & 5:03 & 4:56 & 4:51 & 4:47 & $4: 44$ \\
\hline 28 . & $5: 48$ & $5: 38$ & $5: 28$ & $5: 20$ & $5: 13$ & $5: 08$ & 5:04 & $5: 01$ \\
\hline 30. & $6: 06$ & 5:56 & $5: 46$ & $5: 37$ & $5: 30$ & $5: 25$ & $5: 21$ & $5: 18$ \\
\hline 32. & $6: 23$ & $6: 13$ & $6: 03$ & $5: 54$ & $5: 47$ & $5: 42$ & $5: 38$ & $5: 35$ \\
\hline 34. & $6: 40$ & $6: 30$ & $6: 20$ & $6: 11$ & $6: 04$ & $5: 59$ & $5: 55$ & 5:52 \\
\hline 36. & $6: 56$ & $6: 46$ & $6: 37$ & $6: 28$ & $6: 21$ & $6: 17$ & $6: 12$ & $6: 08$ \\
\hline 38. & $7: 13$ & 7:03 & $6: 54$ & $6: 45$ & $6: 39$ & $6: 34$ & $6: 28$ & $6: 23$ \\
\hline 40. & $7: 30$ & $7: 20$ & $7: 11$ & 7:02 & $6: 56$ & $6: 50$ & $6: 44$ & $6: 37$ \\
\hline 42. & $7: 47$ & $7: 36$ & $7: 28$ & $7: 19$ & $7: 12$ & 7:05 & $6: 59$ & $6: 52$ \\
\hline 44. & $8: 03$ & $7: 52$ & $7: 44$ & $7: 35$ & $7: 27$ & $7: 20$ & $7: 14$ & $7: 07$ \\
\hline 46. & $8: 18$ & $8: 08$ & $7: 59$ & $7: 50$ & $7: 42$ & 7:35 & $7: 29$ & $7: 21$ \\
\hline 48. & $8: 33$ & $8: 23$ & $8: 14$ & $8: 05$ & $7: 57$ & 7:50 & 7:43 & $7: 36$ \\
\hline 50 . & $8: 47$ & $8: 38$ & $8: 28$ & $8: 20$ & $8: 12$ & $8: 05$ & 7:58 & $7: 51$ \\
\hline 52 . & $9: 02$ & $8: 53$ & $8: 43$ & $8: 35$ & $8: 26$ & $8: 19$ & $8: 12$ & $8: 05$ \\
\hline 54. & $9: 17$ & $9: 08$ & $8: 57$ & $8: 49$ & $8: 40$ & $8: 33$ & $8: 27$ & $8: 20$ \\
\hline 56 . & $9: 31$ & $9: 22$ & $9: 12$ & 9:04 & $8: 55$ & $8: 47$ & $8: 41$ & $8: 35$ \\
\hline 58. & $9: 45$ & $9: 35$ & $9: 26$ & $9: 18$ & 9:09 & $9: 01$ & $8: 55$ & $8: 49$ \\
\hline 60. & $10: 00$ & $9: 49$ & $9: 40$ & $9: 31$ & $9: 22$ & $9: 15$ & $9: 08$ & $9: 02$ \\
\hline 62 . & $10: 14$ & $10: 03$ & 9:53 & $9: 44$ & $9: 35$ & $9: 28$ & $9: 21$ & $9: 15$ \\
\hline 64. & $10: 28$ & $10: 16$ & $10: 05$ & $9: 57$ & $9: 48$ & $9: 40$ & 9:34 & $9: 28$ \\
\hline 66. & $10: 41$ & $10: 29$ & $10: 18$ & $10: 09$ & 10:01 & $9: 53$ & $9: 47$ & $9: 41$ \\
\hline 68. & $10: 54$ & $10: 42$ & $10: 31$ & $10: 22$ & $10: 13$ & $10: 06$ & $9: 59$ & 9:53 \\
\hline 70. & $11: 07$ & $10: 55$ & $10: 44$ & $10: 35$ & $10: 26$ & $10: 18$ & 10:11 & $10: 05$ \\
\hline
\end{tabular}


TABLE 6-(Concluded)

\begin{tabular}{|c|c|c|c|c|c|c|c|c|}
\hline \multirow{2}{*}{$\begin{array}{l}\text { Distance } \\
\text { in deg. }\end{array}$} & \multicolumn{8}{|c|}{ Depth of focus in $\mathrm{km}$. } \\
\hline & 100 & 200 & 300 & 400 & 500 & 600 & 700 & 800 \\
\hline 72 . & $11: 19$ & 11:07 & $10: 55$ & $10: 46$ & $10: 37$ & $10: 29$ & $10: 22$ & $10: 15$ \\
\hline $74 .$. & $11: 30$ & $11: 18$ & $11: 06$ & $10: 57$ & $10: 48$ & $10: 40$ & $10: 33$ & $10: 26$ \\
\hline 76. & $11: 41$ & $11: 29$ & $11: 17$ & $11: 08$ & $10: 59$ & $10: 51$ & $10: 44$ & $10: 36$ \\
\hline $78 .$. & $11: 52$ & $11: 40$ & $11: 29$ & $11: 19$ & $11: 10$ & $11: 02$ & $10: 55$ & $10: 47$ \\
\hline 80. & $12: 03$ & 11:5̃1 & $11: 40$ & $11: 30$ & $11: 21$ & $11: 13$ & $11: 05$ & $10: 57$ \\
\hline 82. & $12: 14$ & $12: 02$ & 11:51 & $11: 40$ & $11: 31$ & $11: 23$ & $11: 15$ & $11: 07$ \\
\hline 84. & $12: 24$ & $12: 12$ & $12: 01$ & $11: 50$ & 11:41 & $11: 33$ & $11: 25$ & $11: 17$ \\
\hline 86 . & $12: 34$ & $12: 22$ & $12: 11$ & $12: 01$ & $11: 51$ & $11: 42$ & $11: 34$ & $11: 26$ \\
\hline 88. & 12:44 & $12: 31$ & $12: 21$ & $12: 11$ & $12: 01$ & $11: 52$ & $11: 43$ & $11: 35$ \\
\hline 90. & $12: 53$ & $12: 41$ & $12: 30$ & $12: 20$ & $12: 10$ & $12: 01$ & $11: 52$ & $11: 44$ \\
\hline 92. & $13: 02$ & $12: 50$ & $12: 39$ & $12: 29$ & $12: 19$ & $12: 10$ & 12:01 & $11: 53$ \\
\hline 94. & $13: 11$ & $12: 59$ & $12: 48$ & $12: 38$ & $12: 28$ & $12: 19$ & $12: 10$ & 12:02 \\
\hline 96. & $13: 20$ & $13: 08$ & $12: 57$ & $12: 47$ & $12: 37$ & $12: 28$ & $12: 19$ & $12: 11$ \\
\hline 98. & 13:28 & $13: 17$ & $13: 06$ & $12: 56$ & $12: 46$ & $12: 37$ & $12: 28$ & $12: 20$ \\
\hline 100. & $13: 37$ & $13: 25$ & 13:14 & $13: 04$ & $12: 55$ & $12: 46$ & $12: 37$ & $12: 29$ \\
\hline 102. & $13: 46$ & $13: 34$ & $13: 23$ & $13: 13$ & $13: 04$ & $12: 55$ & $12: 46$ & $12: 37$ \\
\hline 104. & $13: 55$ & $13: 43$ & $13: 32$ & $13: 22$ & $13: 13$ & 13:04 & $12: 55$ & $12: 46$ \\
\hline 106. & 14:04 & $13: 52$ & 13:41 & $13: 31$ & $13: 22$ & $13: 13$ & $13: 04$ & $12: 56$ \\
\hline 108. & 14:13 & $14: 01$ & $13: 50$ & $13: 40$ & $13: 30$ & $13: 21$ & $13: 13$ & $13: 05$ \\
\hline $110 \ldots$ & $14: 22$ & $14: 10$ & $13: 59$ & $13: 49$ & $13: 39$ & $13: 30$ & $13: 22$ & $13: 14$ \\
\hline $115 \ldots$ & $14: 44$ & $14: 32$ & $14: 21$ & $14: 11$ & 14:01 & $13: 52$ & $13: 43$ & $13: 35$ \\
\hline 120. & $15: 06$ & $14: 54$ & $14: 43$ & $14: 33$ & $14: 23$ & $14: 14$ & 14:05 & $13: 57$ \\
\hline $125 \ldots$ & $15: 28$ & $15: 16$ & $15: 05$ & 14:55 & 14:45 & $14: 36$ & 14:27 & $14: 19$ \\
\hline $130 \ldots$ & $15: 51$ & $15: 39$ & $15: 28$ & $15: 18$ & $15: 08$ & $14: 59$ & $14: 50$ & $14: 42$ \\
\hline 140. & $16: 36$ & $16: 24$ & $16: 13$ & $16: 03$ & $15: 53$ & $15: 44$ & $15: 35$ & $15: 27$ \\
\hline 150. & $17: 21$ & $17: 09$ & $16: 58$ & $16: 48$ & $16: 38$ & $16: 29$ & $16: 20$ & $16: 12$ \\
\hline
\end{tabular}

pP and PP begin together at a critical distance which increases with depth of focus. At shorter distances neither phase exists, except possibly as a result of diffraction or of irregularity of the reflecting surface. Near the critical distance the calculated times are much affected by slight changes in the assumptions; our tabulated times, and the corresponding critical distances, are consequently rather uncertain. Contrary to what has occasionally been supposed, the critical point is not a focal point (caustic). In the case of a true focal point like that of $\mathrm{P}^{\prime}, \frac{d i}{d \Delta}$ is infinite, whereas in the present case $\frac{d i}{d \Delta}$ has a finite value. In the first case the amplitudes are theoretically infinite (definition of a focal point), where- 
as in the second case they are finite. However, somewhat increased amplitudes are possible at distances slightly beyond the critical point, as $\mathrm{pP}$ and $\mathrm{PP}$ then arrive close together.

Similar phenomena occur in the case of $\mathrm{pPP}$ and PPP, pS and PS, sS and SS, etc. For $\mathrm{sP}$ and SP, sPP and SPP, etc., conditions are more complicated. There are three distinct cases, all of which depend on the angle of incidence (at the surface of the earth) of the direct $\mathbf{S}$ wave which has left the hypocenter horizontally. This angle, $i^{*}$, corresponds to the point of inflection of the travel-time curve of $\mathrm{S}$.

TABLE 7

Travel Times of $\mathbf{P}^{\prime}$ and $\mathrm{P}_{2}^{\prime}$ in Min.: Sec. and Distance $\Delta^{\prime}$ of the Focus in Degrees

\begin{tabular}{|c|c|c|c|c|c|c|c|c|}
\hline \multirow{2}{*}{$\begin{array}{l}\text { Distance } \\
\text { in deg. }\end{array}$} & \multicolumn{8}{|c|}{ Depth of focus in $\mathrm{km}$. } \\
\hline & 100 & 200 & 300 & 400 & 500 & 600 & 700 & 800 \\
\hline \multicolumn{9}{|c|}{$\mathrm{P}^{\prime}$} \\
\hline 105. & $17: 59$ & $17: 46$ & $17: 34$ & $17: 24$ & $17: 14$ & $17: 05$ & $16: 57$ & $16: 49$ \\
\hline 110. & $18: 16$ & $18: 03$ & $17: 51$ & $17: 41$ & $17: 31$ & $17: 22$ & $17: 14$ & $17: 05$ \\
\hline 115. & $18: 32$ & $18: 19$ & 18:07 & $17: 56$ & $17: 46$ & $17: 37$ & $17: 29$ & $17: 20$ \\
\hline $120 \ldots$ & $18: 45$ & $18: 32$ & $18: 21$ & $18: 10$ & $17: 59$ & $17: 49$ & $17: 40$ & $17: 32$ \\
\hline 125 & $18: 56$ & $18: 44$ & $18: 32$ & $18: 21$ & $18: 11$ & 18:01 & $17: 52$ & $17: 43$ \\
\hline 130 & $19: 05$ & $18: 53$ & $18: 41$ & $18: 30$ & $18: 20$ & $18: 10$ & $18: 01$ & $17: 52$ \\
\hline 135 . & $19: 11$ & $18: 59$ & $18: 47$ & $18: 36$ & $18: 26$ & $18: 16$ & $18: 06$ & $17: 58$ \\
\hline 140. & $19: 16$ & $19: 03$ & $18: 51$ & $18: 40$ & $18: 30$ & $18: 20$ & $18: 10$ & 18:01 \\
\hline 142. & $19: 17$ & $19: 05$ & $18: 53$ & $18: 43$ & $18: 33$ & $18: 24$ & $18: 15$ & $18: 07$ \\
\hline 145. & $19: 27$ & $19: 15$ & $19: 03$ & $18: 52$ & $18: 42$ & $18: 33$ & $18: 24$ & $18: 16$ \\
\hline 150. & $19: 39$ & $19: 26$ & $19: 14$ & $19: 04$ & $18: 53$ & $18: 44$ & $18: 34$ & $18: 26$ \\
\hline 155. & $19: 46$ & $19: 33$ & $19: 22$ & $19: 11$ & 19:00 & $18: 50$ & $18: 41$ & $18: 32$ \\
\hline 160 . & $19: 51$ & $19: 38$ & $19: 26$ & $19: 15$ & 19:04 & $18: 54$ & $18: 45$ & $18: 36$ \\
\hline 170. & $19: 56$ & $19: 43$ & $19: 31$ & $19: 20$ & 19:10 & 19:01 & $18: 51$ & $18: 42$ \\
\hline $180 \ldots$ & $20: 00$ & $19: 47$ & $19: 35$ & $19: 24$ & 19:13 & $19: 04$ & $19: 54$ & $18: 44$ \\
\hline \multicolumn{9}{|c|}{$\Delta^{\prime}$} \\
\hline & 142 & $1413 / 4$ & $1411 / 2$ & $141 \frac{1}{4}$ & 141 & $1403 / 4$ & $1401 / 2$ & $140 \frac{1}{4}$ \\
\hline \multicolumn{9}{|c|}{$\mathrm{P}_{2}^{\prime}$} \\
\hline 150. & $19: 57$ & $19: 45$ & $19: 34$ & $19: 24$ & $19: 15$ & $19: 06$ & $18: 58$ & $18: 50$ \\
\hline 160. & $20: 46$ & $20: 34$ & $20: 23$ & $20: 13$ & $20: 04$ & $19: 55$ & $19: 47$ & $19: 39$ \\
\hline $170 \ldots$ & $21: 34$ & $21: 22$ & $21: 11$ & $21: 01$ & $20: 52$ & $20: 43$ & $20: 35$ & $20: 27$ \\
\hline
\end{tabular}


TABLE 8

Travel Times of $\mathrm{pP}, \mathrm{pP}^{\prime}$, And $\mathrm{pP}^{\prime}{ }_{2}$ in Min.: Sec.

(The line marked "Focus" gives the distance of the focus of $\mathrm{pP}^{\prime}$.)

\begin{tabular}{|c|c|c|c|c|c|c|c|c|}
\hline \multirow{2}{*}{$\begin{array}{l}\text { Distanee } \\
\text { in deg. }\end{array}$} & \multicolumn{8}{|c|}{ Depth of focus in $\mathrm{km}$. } \\
\hline & 100 & 200 & 300 & 400 & 500 & 600 & 700 & 800 \\
\hline \multicolumn{9}{|c|}{$\mathrm{pP}$} \\
\hline 20. & $4: 46$ & $4: 53$ & & & & & & \\
\hline 25 & $5: 40$ & 5:48 & $5: 55$ & $6: 00$ & & & & \\
\hline 30 . & $6: 25$ & $6: 34$ & $6: 43$ & $6: 50$ & & & & \\
\hline 35. & 7:09 & 7:19 & $7: 28$ & $7: 35$ & 7:40 & $7: 45$ & & \\
\hline 40 . & $7: 53$ & 8:03 & $8: 11$ & $8: 18$ & $8: 25$ & $8: 30$ & & \\
\hline 45. & $8: 35$ & $8: 45$ & $8: 54$ & 9:01 & 9:07 & $9: 14$ & $9: 19$ & \\
\hline 50 & $9: 13$ & $9: 23$ & 9:33 & $9: 41$ & 9:48 & $9: 55$ & 10:01 & $10: 05$ \\
\hline 55 & $9: 49$ & $10: 00$ & $10: 10$ & $10: 18$ & $10: 27$ & $10: 34$ & $10: 40$ & $10: 45$ \\
\hline 60 & $10: 25$ & $10: 36$ & $10: 47$ & $10: 56$ & 11:03 & $11: 10$ & $11: 16$ & $11: 21$ \\
\hline 65 & $11: 00$ & $11: 11$ & $11: 22$ & $11: 31$ & $11: 39$ & $11: 46$ & 11:52 & $11: 57$ \\
\hline 70 . & $11: 32$ & $11: 43$ & $11: 54$ & $12: 04$ & $12: 13$ & $12: 20$ & $11: 26$ & $12: 33$ \\
\hline 75 & $12: 01$ & $12: 13$ & $12: 24$ & $12: 34$ & $12: 43$ & $12: 50$ & $12: 57$ & $13: 04$ \\
\hline $80 \ldots$ & $12: 29$ & $12: 41$ & 12:53 & $13: 03$ & $13: 12$ & $13: 20$ & $13: 27$ & $13: 34$ \\
\hline 85 & $12: 55$ & $13: 07$ & $13: 18$ & $13: 28$ & $13: 38$ & $13: 46$ & 13:54 & $14: 01$ \\
\hline 90 & $13: 20$ & $13: 31$ & $13: 42$ & $13: 53$ & $14: 03$ & $14: 12$ & $14: 20$ & $14: 27$ \\
\hline 100. & 14:04 & $14: 16$ & $14: 27$ & $14: 37$ & $14: 46$ & $14: 55$ & 15:04 & $15: 12$ \\
\hline 110. & $14: 48$ & $15: 00$ & $15: 11$ & $15: 22$ & $15: 31$ & $15: 39$ & $15: 48$ & $15: 56$ \\
\hline 120. & $15: 32$ & $15: 44$ & $15: 56$ & $16: 06$ & $16: 15$ & $16: 23$ & $16: 32$ & $16: 40$ \\
\hline 130 & $16: 17$ & $16: 29$ & $16: 41$ & $16: 51$ & $17: 00$ & $17: 08$ & $17: 17$ & $17: 25$ \\
\hline 140. & $17: 01$ & $17: 13$ & $17: 25$ & $17: 35$ & $17: 44$ & $17: 53$ & $18: 02$ & $18: 10$ \\
\hline \multicolumn{9}{|c|}{$\mathrm{p} \mathbf{P}^{\prime}$} \\
\hline 120. & $19: 12$ & $19: 25$ & $19: 37$ & $19: 48$ & $19: 58$ & $20: 07$ & $20: 16$ & $20: 25$ \\
\hline 130. & $19: 32$ & $19: 45$ & $19: 57$ & $20: 09$ & $20: 20$ & $20: 29$ & $20: 38$ & $20: 46$ \\
\hline 140. & $19: 44$ & $19: 57$ & $20: 10$ & $20: 21$ & $20: 32$ & $20: 42$ & $20: 51$ & $21: 00$ \\
\hline 145. & $19: 56$ & $20: 08$ & $20: 19$ & $20: 30$ & $20: 40$ & $20: 50$ & $20: 59$ & $21: 08$ \\
\hline 150. & $20: 08$ & $20: 20$ & $20: 32$ & $20: 43$ & $20: 53$ & $21: 03$ & $21: 12$ & $21: 21$ \\
\hline 160. & $20: 19$ & $20: 32$ & $20: 44$ & $20: 55$ & $21: 05$ & $21: 15$ & $21: 25$ & $21: 34$ \\
\hline \multirow{3}{*}{180.} & $20: 27$ & $20: 40$ & $20: 52$ & $21: 03$ & $21: 13$ & $21: 23$ & $21: 34$ & $21: 43$ \\
\hline & \multicolumn{8}{|c|}{ Focus } \\
\hline & $1421 / 4$ & $142 \frac{1}{2}$ & $142 \frac{1}{2}$ & $1423 / 4$ & 143 & $1431 / 4$ & $1431 / 2$ & $1433 / 4$ \\
\hline \multicolumn{9}{|c|}{$\mathrm{pP}_{2}^{\prime}$} \\
\hline 150 . & $20: 24$ & $20: 36$ & $20: 47$ & $20: 57$ & $21: 06$ & $21: 15$ & $21: 23$ & $21: 31$ \\
\hline 160. & 21:13 & $21: 25$ & $21: 36$ & $21: 46$ & $21: 55$ & $22: 04$ & $22: 12$ & $22: 20$ \\
\hline 170. & 22:00 & $22: 12$ & $22: 23$ & $22: 33$ & $22: 42$ & $22: 51$ & $22: 59$ & $23: 07$ \\
\hline
\end{tabular}


TABLE 9

Travel Times of $\mathrm{sP}, \mathrm{sP}^{\prime}$, And $\mathrm{sP}_{2}^{\prime}$ in Min.: Sec.

(The focus of $\mathrm{sP}^{\prime}$ occurs in about the same distance as the focus of $\mathrm{pP}^{\prime}$.)

\begin{tabular}{|c|c|c|c|c|c|c|c|c|}
\hline \multirow{2}{*}{$\begin{array}{l}\text { Distance } \\
\text { in deg. }\end{array}$} & \multicolumn{8}{|c|}{ Depth of focus in km. } \\
\hline & 100 & 200 & 300 & 400 & 500 & 600 & 700 & 800 \\
\hline \multicolumn{9}{|c|}{$\mathrm{sP}$} \\
\hline 20. & $5: 00$ & $5: 21$ & $5: 40$ & $5: 56$ & $6: 11$ & $6: 22$ & & \\
\hline 25. & $5: 50$ & $6: 10$ & $6: 29$ & $6: 47$ & $7: 05$ & $7: 20$ & $7: 34$ & $7: 47$ \\
\hline 30. & $6: 39$ & $7: 00$ & $7: 18$ & $7: 36$ & $7: 54$ & $8: 10$ & $8: 24$ & $8: 37$ \\
\hline 35 . & $7: 23$ & $7: 43$ & $8: 02$ & $8: 20$ & $8: 38$ & $8: 55$ & $9: 11$ & $9: 26$ \\
\hline 40. & $8: 07$ & $8: 27$ & $8: 46$ & $9: 04$ & $9: 22$ & $9: 39$ & $9: 55$ & $10: 09$ \\
\hline 45. & $8: 47$ & $9: 08$ & $9: 27$ & $9: 46$ & $10: 04$ & $10: 21$ & $10: 35$ & $10: 49$ \\
\hline 50 . & $9: 26$ & $9: 46$ & $10: 05$ & $10: 23$ & $10: 41$ & $10: 59$ & $11: 15$ & $11: 30$ \\
\hline 55 & $10: 01$ & $10: 22$ & $10: 42$ & $11: 01$ & $11: 19$ & $11: 36$ & $11: 53$ & $12: 08$ \\
\hline 60. & $10: 36$ & $10: 57$ & $11: 16$ & $11: 35$ & $11: 54$ & $12: 12$ & $12: 29$ & $12: 44$ \\
\hline 65. & $11: 10$ & $11: 31$ & 11:51 & $12: 10$ & $12: 29$ & $12: 47$ & $13: 04$ & $13: 18$ \\
\hline 70 . & $11: 43$ & $12: 05$ & $12: 25$ & $12: 44$ & $13: 03$ & $13: 21$ & $13: 39$ & $13: 53$ \\
\hline 75 . & $12: 12$ & $12: 34$ & $12: 54$ & $13: 15$ & $13: 33$ & $13: 51$ & $14: 08$ & $14: 25$ \\
\hline 80. & $12: 40$ & 13:03 & $13: 24$ & $13: 44$ & $14: 03$ & $14: 20$ & $14: 36$ & $14: 51$ \\
\hline $85 .$. & $13: 06$ & $13: 29$ & $13: 50$ & 14:10 & $14: 28$ & $14: 45$ & $15: 02$ & $15: 19$ \\
\hline $90 \ldots$ & $13: 30$ & $13: 53$ & 14:14 & $14: 34$ & $14: 53$ & $15: 11$ & $15: 28$ & $15: 43$ \\
\hline 100. & $14: 14$ & $14: 37$ & $14: 58$ & $15: 18$ & $15: 37$ & $15: 55$ & $16: 12$ & $16: 28$ \\
\hline 110. & $14: 59$ & $15: 22$ & $15: 43$ & $16: 03$ & $16: 22$ & $16: 40$ & $16: 57$ & $17: 13$ \\
\hline 120. & $15: 44$ & $16: 07$ & $16: 28$ & $16: 48$ & $17: 07$ & $17: 25$ & $17: 42$ & $17: 58$ \\
\hline 130. & $16: 29$ & $16: 52$ & $17: 13$ & $17: 33$ & $17: 52$ & $18: 10$ & $18: 27$ & $18: 43$ \\
\hline 140. & $17: 14$ & $17: 37$ & $17: 58$ & $18: 18$ & $18: 37$ & $18: 55$ & 19:12 & $19: 28$ \\
\hline \multicolumn{9}{|c|}{$s \mathrm{P}^{\prime}$} \\
\hline 130. & $19: 41$ & $20: 04$ & $20: 26$ & $20: 46$ & 21:05 & $21: 24$ & $21: 41$ & $21: 58$ \\
\hline 140. & $19: 53$ & $20: 17$ & $20: 39$ & $20: 59$ & 21:18 & $21: 37$ & $21: 54$ & $22: 11$ \\
\hline 145. & $20: 05$ & $20: 28$ & $20: 50$ & $21: 10$ & $21: 29$ & $21: 48$ & $22: 06$ & $22: 24$ \\
\hline 150. & $20: 18$ & $20: 41$ & $21: 03$ & $21: 23$ & $21: 42$ & $22: 01$ & $22: 19$ & $22: 36$ \\
\hline 160. & $20: 29$ & $20: 52$ & $21: 14$ & $21: 34$ & $21: 53$ & $22: 12$ & $22: 30$ & $22: 47$ \\
\hline $180 \ldots \ldots$ & $20: 37$ & 21:00 & $21: 22$ & $21: 42$ & 22:01 & $22: 20$ & $22: 38$ & $22: 55$ \\
\hline \multicolumn{9}{|c|}{$\mathrm{sP}_{2}^{\prime}$} \\
\hline 150. & $20: 34$ & $20: 56$ & $21: 17$ & $21: 37$ & $21: 56$ & $22: 14$ & $22: 31$ & $22: 48$ \\
\hline 160. & $21: 23$ & $21: 45$ & 22:06 & $22: 26$ & $22: 45$ & 23:02 & $23: 19$ & $23: 35$ \\
\hline 170. & $22: 10$ & $22: 32$ & $22: 53$ & $23: 13$ & $23: 32$ & $23: 49$ & 24:06 & $24: 22$ \\
\hline
\end{tabular}


The angle $i^{*}$ is a function of the focal depth; and there exists a particular critical depth, $h^{\prime}$, which is such that $\sin i^{*}=\frac{v}{V}$, where $v$ and $V$ are the velocities of transverse and longitudinal waves in the surface layer. In the case where the focus is at this critical depth, $\left(h=h^{\prime}\right)$, the travel-time curves of $\mathrm{sP}, \mathrm{sPP}, \mathrm{SP}$, SPP, etc., all begin at the point of inflection of the $\mathrm{S}$ curve.

If $\sin i^{*}<\frac{v}{V}\left(h>h^{\prime}\right), \mathrm{sP}$ and $\mathrm{SP}$ begin at a common point in a way similar to that for $\mathrm{pP}$ and PP; sPP and SPP begin at another common point, etc. These points are not on the $S$ curve.

If $\sin i^{*}>\frac{v}{V}\left(h<h^{\prime}\right), \mathrm{sP}, \mathrm{sPP}$, etc., begin together on the $\mathrm{S}$ curve at a distance TABLE 10

Travel Times of PP in Min.: Sec.

\begin{tabular}{|c|c|c|c|c|c|c|c|c|}
\hline \multirow{2}{*}{$\begin{array}{l}\text { Distance } \\
\text { in deg. }\end{array}$} & \multicolumn{8}{|c|}{ Depth of focus in $\mathrm{km}$. } \\
\hline & 100 & 200 & 300 & 400 & 500 & 600 & 700 & 800 \\
\hline 20. & $5: 02$ & & & & & & & \\
\hline$\ldots \ldots \ldots$ & $6: 05$ & $6: 03$ & $6: 02$ & $6: 01$ & & & & \\
\hline 30. & $7: 08$ & $7: 05$ & 7:02 & $6: 58$ & & & & \\
\hline 35. & $8: 11$ & $8: 07$ & $8: 02$ & $7: 58$ & $7: 54$ & $7: 51$ & & \\
\hline 40. & $9: 07$ & $8: 59$ & $8: 52$ & $8: 46$ & $8: 42$ & $8: 39$ & $8: 37$ & \\
\hline 45. & $9: 58$ & $9: 50$ & $9: 42$ & $9: 35$ & 9:30 & $9: 26$ & $9: 23$ & \\
\hline 50. & $10: 47$ & $10: 38$ & $10: 30$ & $10: 23$ & $10: 18$ & $10: 14$ & $10: 10$ & $10: 07$ \\
\hline 55. & $11: 33$ & $11: 25$ & $11: 17$ & $11: 09$ & $11: 03$ & $10: 58$ & $10: 54$ & $10: 51$ \\
\hline $60 \ldots \ldots$ & $12: 18$ & $12: 09$ & $12: 01$ & $11: 53$ & $11: 46$ & $11: 41$ & $11: 38$ & $11: 35$ \\
\hline $65 \ldots \ldots \ldots \ldots$ & $13: 02$ & $12: 53$ & $12: 44$ & $12: 36$ & $12: 29$ & $12: 25$ & $12: 21$ & $12: 18$ \\
\hline 70 & $13: 46$ & $13: 37$ & $13: 28$ & $13: 20$ & $13: 13$ & $13: 08$ & $13: 04$ & $13: 01$ \\
\hline 75. & $14: 30$ & $14: 20$ & $14: 11$ & $14: 03$ & $13: 56$ & $13: 51$ & $13: 47$ & $13: 44$ \\
\hline 80. & $15: 13$ & $15: 02$ & $14: 53$ & $14: 45$ & $14: 38$ & $14: 33$ & $14: 29$ & $14: 25$ \\
\hline 85. & $15: 54$ & $15: 43$ & $15: 33$ & $15: 25$ & $15: 17$ & $15: 11$ & $15: 06$ & $15: 02$ \\
\hline 90. & $16: 33$ & $16: 23$ & $16: 13$ & $16: 04$ & $15: 56$ & $15: 48$ & $15: 42$ & $15: 37$ \\
\hline 100. & $17: 47$ & $17: 37$ & $17: 27$ & $17: 18$ & $17: 10$ & $17: 01$ & $16: 55$ & $16: 49$ \\
\hline $110 \ldots \ldots \ldots \ldots$ & $18: 59$ & $18: 48$ & 18:38 & $18: 29$ & $18: 20$ & $18: 12$ & $18: 05$ & $17: 59$ \\
\hline $120 \ldots \ldots \ldots \ldots$ & $20: 10$ & $19: 59$ & $19: 48$ & $19: 39$ & $19: 30$ & $19: 22$ & $19: 15$ & $19: 09$ \\
\hline $130 \ldots \ldots$ & $21: 20$ & $21: 09$ & $20: 58$ & $20: 48$ & $20: 39$ & $20: 31$ & $20: 24$ & $20: 18$ \\
\hline 140. & $22: 26$ & $22: 14$ & $22: 03$ & $21: 53$ & $21: 45$ & $21: 37$ & $21: 30$ & $21: 23$ \\
\hline 150. & $23: 24$ & $23: 11$ & $23: 00$ & $22: 50$ & $22: 41$ & $22: 33$ & $22: 24$ & $22: 18$ \\
\hline 160. & $24: 18$ & $24: 05$ & $23: 54$ & $23: 45$ & $23: 36$ & $23: 28$ & $23: 19$ & $23: 12$ \\
\hline $170 \ldots \ldots$ & $25: 11$ & $24: 59$ & $24: 48$ & $24: 38$ & $24: 29$ & $24: 20$ & $24: 11$ & 24:04 \\
\hline $180 \ldots \ldots$ & $26: 02$ & $25: 50$ & $25: 39$ & $25: 29$ & $25: 20$ & $25: 10$ & 25:01 & $24: 53$ \\
\hline
\end{tabular}


TABLE 11

Travel Times of pPP in Min.: Sec,

\begin{tabular}{|c|c|c|c|c|c|c|c|c|}
\hline \multirow{2}{*}{$\begin{array}{l}\text { Distance } \\
\text { in deg. }\end{array}$} & \multicolumn{8}{|c|}{ Depth of focus in $\mathrm{km}$. } \\
\hline & 100 & 200 & 300 & 400 & 500 & 600 & 700 & 800 \\
\hline $40 \ldots \ldots \ldots$ & $9: 26$ & $9: 34$ & & & & & & \\
\hline $50 \ldots \ldots \ldots$ & $11: 05$ & $11: 15$ & $11: 22$ & $11: 24$ & & & & \\
\hline $60 \ldots \ldots$ & $12: 39$ & $12: 50$ & $12: 58$ & $13: 04$ & $13: 08$ & $13: 11$ & & \\
\hline $70 \ldots \ldots \ldots$ & $14: 07$ & $14: 17$ & $14: 26$ & $14: 38$ & $14: 38$ & $14: 44$ & $14: 47$ & \\
\hline 80 & $15: 35$ & $15: 46$ & $15: 55$ & $16: 02$ & $16: 08$ & $16: 13$ & $16: 16$ & $16: 17$ \\
\hline 90. & $16: 55$ & $17: 06$ & $17: 15$ & $17: 23$ & $17: 30$ & $17: 35$ & $17: 40$ & $17: 44$ \\
\hline $100 \ldots \ldots \ldots \ldots$ & $18: 11$ & $18: 22$ & $18: 31$ & $18: 40$ & $18: 47$ & $18: 54$ & $18: 59$ & $19: 03$ \\
\hline $110 \ldots \ldots \ldots \ldots$ & $19: 23$ & $19: 34$ & $19: 43$ & $19: 53$ & $20: 01$ & $20: 07$ & $20: 13$ & $20: 18$ \\
\hline 120 . & $20: 35$ & $20: 46$ & $20: 55$ & $21: 04$ & $21: 13$ & $21: 20$ & $21: 26$ & $21: 31$ \\
\hline $130 \ldots \ldots$ & $21: 45$ & $21: 56$ & $22: 05$ & $22: 13$ & $22: 22$ & $22: 30$ & $22: 36$ & $22: 42$ \\
\hline $140 \ldots \ldots \ldots \ldots$ & $22: 50$ & $23: 02$ & $23: 13$ & $23: 22$ & $23: 31$ & $23: 38$ & $23: 45$ & $23: 52$ \\
\hline $150 \ldots \ldots \ldots$ & $23: 48$ & 24:00 & $24: 11$ & $24: 21$ & $24: 30$ & $24: 37$ & $24: 44$ & $24: 51$ \\
\hline 160. & $24: 43$ & $24: 56$ & $25: 07$ & $25: 17$ & $25: 26$ & $25: 34$ & $25: 41$ & $25: 48$ \\
\hline 170. & $25: 36$ & $25: 48$ & $25: 59$ & $26: 09$ & $26: 18$ & $26: 27$ & $26: 34$ & $26: 41$ \\
\hline $180 \ldots \ldots$ & $26: 26$ & $26: 38$ & $26: 50$ & $27: 00$ & $27: 09$ & $27: 18$ & $27: 26$ & $27: 34$ \\
\hline
\end{tabular}

TABLE 12

Travel Times of sPP in Min.: Sec.

\begin{tabular}{|c|c|c|c|c|c|c|c|c|}
\hline \multirow{2}{*}{$\begin{array}{l}\text { Distance } \\
\text { in deg. }\end{array}$} & \multicolumn{8}{|c|}{ Depth of focus in $\mathrm{km}$. } \\
\hline & 100 & 200 & 300 & 400 & 500 & 600 & 700 & 800 \\
\hline $30 \ldots$ & $7: 34$ & $7: 54$ & $8: 10$ & $8: 20$ & $8: 28$ & & & \\
\hline 40 & $9: 40$ & $10: 01$ & $10: 18$ & $10: 33$ & $10: 49$ & $11: 02$ & $11: 14$ & $11: 24$ \\
\hline 50 & $11: 19$ & $11: 40$ & $11: 59$ & $12: 17$ & $12: 35$ & $12: 51$ & $13: 05$ & $13: 21$ \\
\hline 60 & $12: 54$ & $13: 15$ & $13: 34$ & $13: 51$ & $14: 09$ & $14: 25$ & $14: 41$ & $14: 56$ \\
\hline 70 & $14: 22$ & $14: 42$ & $15: 01$ & $15: 19$ & $15: 37$ & $15: 54$ & $16: 09$ & $16: 24$ \\
\hline 80 & $15: 50$ & $16: 10$ & $16: 29$ & $16: 47$ & $17: 05$ & $17: 22$ & $17: 36$ & $17: 51$ \\
\hline 90 & $17: 09$ & $17: 29$ & $17: 48$ & $18: 08$ & $18: 28$ & $18: 44$ & $18: 58$ & $19: 12$ \\
\hline 100 . & $18: 26$ & $18: 45$ & $19: 05$ & $19: 25$ & $19: 44$ & $20: 01$ & $20: 16$ & $20: 30$ \\
\hline 110 . & $19: 37$ & $19: 57$ & $20: 17$ & $20: 37$ & $20: 56$ & $21: 13$ & $21: 28$ & $21: 42$ \\
\hline $120 \ldots \ldots \ldots$ & $20: 48$ & $21: 09$ & $21: 30$ & $21: 49$ & $22: 09$ & $22: 26$ & $22: 41$ & $22: 54$ \\
\hline 130 . & $21: 58$ & $22: 20$ & $22: 40$ & $23: 01$ & $23: 20$ & $23: 39$ & $23: 53$ & $24: 06$ \\
\hline 140 . & $23: 04$ & $23: 28$ & $23: 48$ & $24: 09$ & $24: 29$ & $24: 47$ & $25: 03$ & $25: 16$ \\
\hline 150. & $24: 01$ & $24: 26$ & $24: 46$ & $25: 07$ & $25: 26$ & $25: 45$ & $26: 02$ & $26: 15$ \\
\hline 160 . & $24: 56$ & $25: 20$ & $25: 41$ & $26: 03$ & $26: 21$ & $26: 40$ & $26: 57$ & $27: 11$ \\
\hline 170 . & $25: 47$ & $26: 11$ & $26: 32$ & $26: 54$ & $27: 12$ & $27: 30$ & $27: 48$ & $28: 02$ \\
\hline 180 . & $26: 37$ & $27: 00$ & $27: 22$ & $27: 43$ & $28: 01$ & $28: 19$ & $28: 37$ & $28: 52$ \\
\hline
\end{tabular}


less than that of the point of inflection, and SP, SPP, etc., begin together at a second point more distant than the point of inflection, and also on the $\mathbf{S}$ curve.

The critical depth $h^{\prime}$, and the travel-time curves themselves, depend very much on the crustal structure. For continental structure, $h^{\prime}$ is of the order of $500 \mathrm{~km}$. For Pacific structure $h^{\prime}$ is considerably greater.

The travel times for $\mathrm{S}$ as given in table 15 are tentative only; they are based on the times for normal shocks, as settled upon in "On Seismic Waves, I and II." Results discussed below (p. 387) indicate that these times may require further revision; this will affect all travel-time curves for phases involving a

TABLE 13

Travel Times of PPP in Min.: Sec.

\begin{tabular}{|c|c|c|c|c|c|c|c|c|}
\hline \multirow{2}{*}{$\begin{array}{l}\text { Distance } \\
\text { in deg. }\end{array}$} & \multicolumn{8}{|c|}{ Depth of focus in km. } \\
\hline & 100 & 200 & 300 & 400 & 500 & 600 & 700 & 800 \\
\hline 40. & $9: 42$ & $9: 40$ & $9: 39$ & & & & & \\
\hline 50. & $11: 44$ & $11: 39$ & $11: 35$ & $11: 32$ & & & & \\
\hline 60. & $13: 42$ & $13: 35$ & $13: 30$ & $13: 26$ & $13: 23$ & $13: 20$ & & \\
\hline 70. & $15: 27$ & $15: 17$ & $15: 09$ & $15: 03$ & 14:58 & $14: 54$ & $14: 51$ & \\
\hline 80. & $17: 02$ & $16: 53$ & $16: 42$ & $16: 37$ & $16: 32$ & $16: 28$ & $16: 26$ & $16: 25$ \\
\hline $90 .$. & $18: 32$ & $18: 23$ & $18: 14$ & 18:07 & 18:02 & $17: 58$ & $17: 56$ & $17: 55$ \\
\hline 100. & 20:01 & $19: 52$ & $19: 43$ & $19: 34$ & $19: 29$ & $19: 24$ & 19:21 & $19: 19$ \\
\hline 120 . & 22.55 & $22: 45$ & $22: 35$ & $22: 26$ & $22: 20$ & $22: 14$ & $22: 10$ & $22: 06$ \\
\hline 140. & $25: 36$ & $25: 25$ & $25: 16$ & $25: 07$ & $24: 58$ & 24:50 & $24: 44$ & $24: 38$ \\
\hline $160 .$. & 28:01 & $27: 51$ & $27: 41$ & $27: 32$ & $27: 24$ & $27: 17$ & $27: 10$ & $27: 04$ \\
\hline 180. & $30: 22$ & $30: 11$ & $30: 01$ & $29: 51$ & $29: 43$ & $29: 36$ & $29: 29$ & $29: 22$ \\
\hline
\end{tabular}

TABLE 14

Travel Times of pPPP in Min.: Sec.

\begin{tabular}{r|c|c|c|c|c|c|c|c}
\hline \multirow{2}{*}{$\begin{array}{c}\text { } \\
\text { Distance } \\
\text { in deg. }\end{array}$} & \multicolumn{10}{|c|}{ Depth of focus in km. } \\
\cline { 2 - 9 } & 100 & 200 & 300 & 400 & 500 & 600 & 700 & 800 \\
\hline $60 \ldots \ldots \ldots \ldots \ldots$ & $13: 59$ & $14: 05$ & $14: 09$ & $14: 10$ & & & & \\
$70 \ldots \ldots \ldots \ldots$ & $15: 44$ & $15: 52$ & $15: 57$ & $16: 02$ & $16: 03$ & & & \\
$80 \ldots \ldots \ldots \ldots$ & $17: 23$ & $17: 32$ & $17: 40$ & $17: 46$ & $17: 48$ & & & \\
$90 \ldots \ldots \ldots \ldots \ldots$ & $18: 54$ & $19: 03$ & $19: 11$ & $19: 17$ & $19: 23$ & $19: 25$ & & \\
$100 \ldots \ldots \ldots \ldots \ldots$ & $20: 22$ & $20: 31$ & $20: 40$ & $20: 47$ & $20: 53$ & $20: 56$ & $20: 57$ & \\
$120 \ldots \ldots \ldots \ldots \ldots$ & $23: 16$ & $23: 27$ & $23: 35$ & $23: 43$ & $23: 49$ & $23: 54$ & $23: 57$ & $23: 59$ \\
$140 \ldots \ldots \ldots \ldots$ & $25: 59$ & $26: 10$ & $26: 19$ & $26: 27$ & $26: 34$ & $26: 40$ & $26: 46$ & $26: 51$ \\
$160 \ldots \ldots \ldots \ldots$ & $28: 24$ & $28: 35$ & $28: 45$ & $28: 55$ & $29: 03$ & $29: 10$ & $29: 17$ & $29: 24$ \\
$180 \ldots \ldots \ldots \ldots \ldots$ & $30: 44$ & $30: 55$ & $31: 06$ & $31: 16$ & $31: 25$ & $31: 32$ & $31: 39$ & $31: 46$ \\
\hline
\end{tabular}


TABLE 15

Traver Trmes of $\mathbf{S}$ in Mrn.: Sec.

[To be revised]

\begin{tabular}{|c|c|c|c|c|c|c|c|c|}
\hline \multirow{2}{*}{$\begin{array}{l}\text { Distance } \\
\text { in deg. }\end{array}$} & \multicolumn{8}{|c|}{ Depth of focus in $\mathrm{km}$. } \\
\hline & 100 & 200 & 300 & 400 & 500 & 600 & 700 & 800 \\
\hline 0. & $0: 24$ & $0: 46$ & $1: 08$ & $1: 29$ & $1: 49$ & $2: 07$ & $2: 24$ & $2: 41$ \\
\hline 2. & $1: 00$ & $1: 06$ & $1: 24$ & $1: 41$ & $1: 59$ & $2: 15$ & $2: 30$ & $2: 47$ \\
\hline 4. & $1: 49$ & $1: 49$ & $1: 51$ & $2: 05$ & $2: 20$ & $2: 34$ & $2: 48$ & 3:02 \\
\hline 6. & $2: 38$ & $2: 37$ & $2: 34$ & 2:40 & $2: 47$ & $2: 58$ & 3:09 & $3: 21$ \\
\hline 8. & $3: 27$ & $3: 24$ & $3: 18$ & 3:19 & $3: 23$ & $3: 30$ & $3: 38$ & $3: 46$ \\
\hline 10. & $4: 16$ & $4: 12$ & $4: 03$ & 4:00 & 4:01 & $4: 06$ & 4:12 & $4: 18$ \\
\hline 12. & $5: 04$ & $4: 56$ & $4: 47$ & $4: 42$ & $4: 39$ & $4: 42$ & $4: 46$ & $4: 50$ \\
\hline 14. & $5: 49$ & $5: 40$ & $5: 31$ & $5: 23$ & $5: 18$ & $5: 18$ & $5: 20$ & $5: 22$ \\
\hline 16. & $6: 35$ & $6: 25$ & $6: 15$ & $6: 05$ & 5:57 & $5: 54$ & $5: 54$ & $5: 54$ \\
\hline 18. & $7: 21$ & $7: 09$ & $6: 57$ & $6: 46$ & $6: 36$ & $6: 30$ & $6: 28$ & $6: 26$ \\
\hline 20. & $8: 04$ & $7: 52$ & $7: 39$ & $7: 26$ & $7: 15$ & 7:06 & 7:02 & $6: 58$ \\
\hline 22. & $8: 44$ & $8: 32$ & $8: 19$ & $8: 06$ & $7: 54$ & $7: 42$ & $7: 36$ & $7: 30$ \\
\hline 24 . & $9: 21$ & 9:05 & $8: 51$ & $8: 37$ & $8: 26$ & $8: 18$ & 8:09 & 8:01 \\
\hline 26 . & $9: 54$ & $9: 37$ & $9: 22$ & $9: 07$ & $8: 56$ & $8: 47$ & $8: 39$ & $8: 31$ \\
\hline 28. & $10: 27$ & 10:09 & $9: 52$ & 9:37 & $9: 26$ & $9: 16$ & 9:08 & 9:01 \\
\hline 30. & $10: 57$ & $10: 39$ & $10: 23$ & $10: 08$ & $9: 56$ & $9: 46$ & $9: 37$ & $9: 29$ \\
\hline 32. & $11: 28$ & $11: 10$ & $10: 53$ & $10: 38$ & $10: 26$ & $10: 15$ & $10: 06$ & $9: 58$ \\
\hline 34 & 11:58 & $11: 40$ & $11: 23$ & 11:08 & 10:56 & $10: 44$ & $10: 35$ & $10: 26$ \\
\hline $36 \ldots$ & $12: 28$ & $12: 10$ & $11: 53$ & $11: 38$ & $11: 25$ & $11: 13$ & 11:03 & $10: 54$ \\
\hline 38. & $12: 58$ & $12: 40$ & $12: 23$ & $12: 08$ & 11:54 & $11: 42$ & $11: 32$ & $11: 23$ \\
\hline 40. & $13: 27$ & 13:09 & $12: 52$ & $12: 38$ & $12: 23$ & $12: 11$ & 12:01 & $11: 51$ \\
\hline 42. & 13:56 & $13: 37$ & $13: 21$ & 13:07 & 12:52 & $12: 39$ & $12: 30$ & $12: 20$ \\
\hline 44. & $14: 24$ & $14: 06$ & $13: 50$ & $13: 35$ & $13: 20$ & $13: 07$ & $12: 58$ & $12: 48$ \\
\hline 46. & 14:51 & 14:34 & $14: 18$ & 14:03 & $13: 48$ & $13: 35$ & $13: 25$ & $13: 16$ \\
\hline 48. & $15: 19$ & $15: 01$ & $14: 45$ & $14: 30$ & $14: 16$ & $14: 02$ & 13:51 & $13: 42$ \\
\hline 50. & $15: 46$ & $15: 28$ & $15: 12$ & $14: 57$ & 14:43 & $14: 29$ & 14:17 & $14: 08$ \\
\hline 52. & $16: 14$ & $15: 55$ & $15: 38$ & $15: 23$ & $15: 10$ & 14:57 & $14: 44$ & $14: 34$ \\
\hline 54. & $16: 41$ & $16: 22$ & $16: 05$ & 15:50 & $15: 37$ & $15: 24$ & $15: 11$ & $15: 00$ \\
\hline $56 \ldots$ & $17: 08$ & $16: 48$ & $16: 32$ & $16: 16$ & $16: 03$ & $15: 49$ & $15: 37$ & $15: 26$ \\
\hline 58. & $17: 35$ & $17: 15$ & $16: 58$ & $16: 42$ & $16: 28$ & $16: 14$ & $16: 02$ & $15: 50$ \\
\hline 60. & 18:02 & $17: 42$ & $17: 24$ & $17: 07$ & $16: 52$ & $16: 38$ & $16: 26$ & $16: 14$ \\
\hline 62. & $18: 28$ & 18:08 & $17: 49$ & $17: 31$ & $17: 16$ & $17: 02$ & $16: 50$ & $16: 38$ \\
\hline $64 .$. & $18: 52$ & $18: 32$ & 18:12 & $17: 55$ & $17: 40$ & $17: 25$ & $17: 13$ & $17: 01$ \\
\hline $66 \ldots$ & $19: 15$ & $18: 55$ & $18: 35$ & $18: 18$ & $18: 03$ & $17: 48$ & $17: 35$ & $17: 23$ \\
\hline 68. & $19: 38$ & $19: 18$ & $18: 58$ & 18:40 & $18: 25$ & 18:10 & 17:57 & $17: 45$ \\
\hline
\end{tabular}


TABLE $15-($ Concluded $)$

\begin{tabular}{|c|c|c|c|c|c|c|c|c|}
\hline \multirow{2}{*}{$\begin{array}{l}\text { Distance } \\
\text { in deg. }\end{array}$} & \multicolumn{8}{|c|}{ Depth of focus in km. } \\
\hline & 100 & 200 & 300 & 400 & 500 & 600 & 700 & 800 \\
\hline 70 . & $20: 01$ & $19: 40$ & $19: 21$ & $19: 03$ & $18: 48$ & $18: 33$ & $18: 20$ & $18: 08$ \\
\hline 72. & $20: 23$ & $20: 03$ & $19: 43$ & $19: 26$ & $19: 10$ & $18: 56$ & $18: 43$ & $18: 31$ \\
\hline 74. & $20: 45$ & $20: 24$ & $20: 05$ & $19: 48$ & $19: 33$ & $19: 19$ & $19: 06$ & $18: 54$ \\
\hline $76 \ldots \ldots \ldots \ldots$ & $21: 07$ & $20: 46$ & $20: 27$ & $20: 10$ & $19: 55$ & $19: 41$ & $19: 28$ & $19: 16$ \\
\hline $78 \ldots \ldots \ldots$ & $21: 29$ & 21:08 & $20: 49$ & $20: 32$ & $20: 17$ & $20: 02$ & $19: 49$ & $19: 37$ \\
\hline 80. & $21: 51$ & $21: 30$ & $21: 11$ & $20: 54$ & $20: 39$ & $20: 23$ & $20: 10$ & $19: 58$ \\
\hline $82 \ldots \ldots \ldots$ & $22: 13$ & $21: 52$ & $21: 33$ & $21: 16$ & $21: 01$ & $20: 46$ & $20: 31$ & $20: 19$ \\
\hline $84 \ldots \ldots \ldots$ & $22: 35$ & $22: 14$ & $21: 55$ & $21: 38$ & $21: 23$ & 21:08 & $20: 53$ & $20: 39$ \\
\hline 86. & $22: 56$ & $22: 35$ & $22: 16$ & $21: 59$ & $21: 43$ & $21: 27$ & $21: 13$ & $20: 59$ \\
\hline 88. & $23: 16$ & $22: 55$ & $22: 36$ & $22: 18$ & $22: 02$ & $21: 44$ & $21: 30$ & $21: 17$ \\
\hline 90 . & $23: 35$ & $23: 14$ & $22: 55$ & $22: 37$ & $22: 20$ & $22: 03$ & $21: 48$ & $21: 35$ \\
\hline 92. & $23: 53$ & $23: 32$ & $23: 12$ & $22: 55$ & $22: 37$ & $22: 20$ & $22: 05$ & $21: 52$ \\
\hline $94 \ldots$ & $24: 11$ & $23: 50$ & 23:31 & $23: 12$ & $22: 54$ & $22: 37$ & $22: 23$ & $22: 09$ \\
\hline 96. & $24: 28$ & $24: 08$ & $23: 48$ & $23: 30$ & $23: 12$ & $22: 55$ & $22: 40$ & $22: 26$ \\
\hline $98 \ldots$ & $24: 46$ & $24: 25$ & $24: 05$ & $23: 47$ & $23: 29$ & $23: 12$ & $22: 57$ & $22: 44$ \\
\hline $100 \ldots$ & 25:03 & $24: 42$ & $24: 23$ & 24:04 & $23: 46$ & $23: 29$ & $23: 14$ & $23: 01$ \\
\hline 105. & $25: 45$ & $25: 24$ & 25:04 & $24: 46$ & $24: 28$ & $24: 11$ & $23: 56$ & $23: 43$ \\
\hline $110 \ldots$ & $26: 26$ & $26: 05$ & $25: 45$ & $25: 27$ & $25: 09$ & $24: 52$ & $24: 37$ & $24: 24$ \\
\hline 115 & $27: 08$ & $26: 47$ & $26: 27$ & $26: 09$ & $25: 51$ & $25: 34$ & $25: 19$ & $25: 06$ \\
\hline 120 & $27: 50$ & $27: 29$ & $27: 09$ & $26: 51$ & $26: 33$ & $26: 16$ & $26: 01$ & $25: 48$ \\
\hline
\end{tabular}

TABLE 16

Travel Times of pS in Min.: Sec.

\begin{tabular}{|c|c|c|c|c|c|c|c|c|}
\hline \multirow{2}{*}{$\begin{array}{l}\text { Distance } \\
\text { in deg. }\end{array}$} & \multicolumn{8}{|c|}{ Depth of focus in $\mathrm{km}$. } \\
\hline & 100 & 200 & 300 & 400 & 500 & 600 & 700 & 800 \\
\hline $70 \ldots$ & $20: 33$ & $20: 38$ & & & & & & \\
\hline 75 . & $21: 30$ & $21: 38$ & & & & & & \\
\hline 80 . & $22: 23$ & $22: 32$ & $22: 37$ & & & & & \\
\hline 85 . & $23: 16$ & $23: 24$ & $23: 30$ & $23: 33$ & & & & \\
\hline $90 \ldots$ & $24: 06$ & $24: 15$ & $24: 22$ & $24: 27$ & $24: 28$ & & & \\
\hline 95. & $24: 53$ & $25: 03$ & 25:11 & $25: 17$ & $25: 20$ & $25: 21$ & & \\
\hline 100 & $25: 37$ & $25: 47$ & $25: 56$ & $26: 02$ & $26: 06$ & $26: 10$ & & \\
\hline $105 \ldots \ldots$ & $26: 20$ & $26: 30$ & $26: 39$ & $26: 46$ & $26: 51$ & $26: 55$ & $26: 56$ & \\
\hline 110 . & $27: 03$ & $27: 13$ & $27: 22$ & $27: 29$ & $27: 35$ & $27: 40$ & $27: 42$ & $27: 43$ \\
\hline 115 & $27: 45$ & $27: 55$ & $28: 04$ & $28: 11$ & $28: 17$ & $28: 22$ & $28: 26$ & $28: 28$ \\
\hline $120 \ldots$ & $28: 27$ & $28: 37$ & $28: 46$ & $28: 53$ & $28: 59$ & $29: 04$ & $29: 08$ & $29: 10$ \\
\hline
\end{tabular}


transverse wave (sP, PS, SKS, etc.). As far as can be determined, the correction nowhere exceeds ten seconds.

Table 33 gives data for PKKP, including the interval PKKP $-\mathrm{P}$, which is independent of focal depth within a very few seconds, down to the greatest depths tabulated $\left(800 \mathrm{~km}\right.$.) PKKP has a true focal point near $120^{\circ}$; this dis-

TABLE 17

Travel Times of sS in Min.: Sec.

\begin{tabular}{|c|c|c|c|c|c|c|c|c|}
\hline \multirow{2}{*}{$\begin{array}{l}\text { Distance } \\
\text { in deg. }\end{array}$} & \multicolumn{8}{|c|}{ Depth of focus in $\mathrm{km}$. } \\
\hline & 100 & 200 & 300 & 400 & 500 & 600 & 700 & 800 \\
\hline 25 . & $10: 14$ & $10: 26$ & $10: 33$ & & & & & \\
\hline 30. & $11: 35$ & $11: 49$ & 12:02 & $12: 10$ & & & & \\
\hline 35. & $12: 53$ & $13: 08$ & $13: 22$ & $13: 34$ & $13: 44$ & & & \\
\hline 40. & $14: 09$ & $14: 26$ & 14:41 & $14: 55$ & $15: 08$ & $15: 15$ & & \\
\hline 45. & $15: 23$ & $15: 40$ & $15: 55$ & $16: 09$ & $16: 23$ & $16: 32$ & $16: 41$ & $16: 46$ \\
\hline 50. & $16: 31$ & $16: 49$ & $17: 06$ & $17: 20$ & $17: 34$ & $17: 46$ & $17: 58$ & $18: 09$ \\
\hline 55 . & $17: 39$ & $17: 58$ & $18: 16$ & $18: 30$ & $18: 44$ & $18: 57$ & $19: 10$ & $19: 21$ \\
\hline 60. & $18: 46$ & $19: 06$ & $19: 22$ & $19: 37$ & $19: 51$ & $20: 04$ & $20: 17$ & $20: 28$ \\
\hline 65. & $19: 46$ & $20: 06$ & $20: 25$ & $20: 42$ & $20: 58$ & $21: 11$ & $21: 24$ & $21: 34$ \\
\hline 70 & $20: 42$ & $21: 02$ & $21: 21$ & $21: 38$ & $21: 54$ & $22: 09$ & $22: 23$ & $22: 34$ \\
\hline 75. & $21: 37$ & $21: 57$ & $22: 16$ & $22: 33$ & $22: 49$ & $23: 04$ & $23: 17$ & $23: 29$ \\
\hline 80. & $22: 32$ & $22: 52$ & $23: 11$ & $23: 28$ & $23: 44$ & $23: 59$ & $24: 13$ & $24: 25$ \\
\hline 85. & $23: 27$ & $23: 47$ & $24: 06$ & $24: 23$ & $24: 39$ & $24: 54$ & $25: 08$ & $25: 20$ \\
\hline 90. & $24: 21$ & $24: 42$ & $25: 01$ & $25: 18$ & $25: 34$ & $25: 49$ & $26: 03$ & $26: 15$ \\
\hline 95. & $25: 07$ & $25: 28$ & $25: 48$ & $26: 06$ & $26: 24$ & $26: 40$ & $26: 55$ & $27: 07$ \\
\hline 100. & $25: 51$ & $26: 12$ & $26: 31$ & $26: 49$ & $27: 07$ & $27: 24$ & $27: 40$ & $27: 53$ \\
\hline 105. & $26: 32$ & $26: 53$ & $27: 13$ & $27: 31$ & $27: 49$ & $28: 06$ & $28: 22$ & $28: 36$ \\
\hline 110. & $27: 14$ & $27: 35$ & $27: 55$ & $28: 13$ & $28: 31$ & $28: 48$ & $29: 04$ & $29: 18$ \\
\hline 115. & $27: 56$ & $28: 17$ & $28: 37$ & $28: 55$ & $29: 13$ & $29: 30$ & $29: 46$ & $30: 00$ \\
\hline 120. & $28: 37$ & $28: 58$ & $29: 18$ & $29: 36$ & $29: 54$ & $30: 11$ & $30: 27$ & $30: 41$ \\
\hline
\end{tabular}

tance increases slowly with hypocentral depth, being about $124^{\circ}$ for $800 \mathrm{~km}$. The first PKKP should be followed by a wave $(\mathrm{PKKP})_{2}$ related to $\mathrm{PKKP}$ as $\mathrm{P}_{2}^{\prime}$ is related to $\mathrm{P}^{\prime}$. In addition, $\mathrm{pPKKP}$, sPKKP, (pPKKP) $)_{2}$, and (sPKKP) are to be expected; the seismograms generally show continuing short-period motion without clear phases.

SKKP (table 34) is still more complicated; it should be followed by PKKS as well as sSKKP, etc. Even the beginning of the group (which should correspond to the SKKP of the table) is usually not definite on the seismograms.

Table 35 gives the travel times of $\mathrm{P}^{\prime} \mathrm{P}^{\prime}$ (including $\mathrm{P}_{2}^{\prime} \mathrm{P}_{2}^{\prime}$ ) and the intervals $\mathrm{P}^{\prime} \mathrm{P}^{\prime}-\mathrm{P}$. The interval depends on hypocentral depth somewhat more than 
TABLE 18

Travel Times of SS in Min.: Sec.

\begin{tabular}{|c|c|c|c|c|c|c|c|c|}
\hline \multirow{2}{*}{$\begin{array}{l}\text { Distance } \\
\text { in deg. }\end{array}$} & \multicolumn{8}{|c|}{ Depth of foeus in km. } \\
\hline & 100 & 200 & 300 & 400 & 500 & 600 & 700 & 800 \\
\hline 40. & $16: 32$ & $16: 20$ & $16: 10$ & $16: 01$ & $15: 53$ & $15: 46$ & $15: 42$ & \\
\hline 50. & $19: 31$ & $19: 15$ & $19: 01$ & $18: 49$ & $18: 39$ & $18: 31$ & $18: 24$ & $18: 20$ \\
\hline 60. & $22: 10$ & $21: 53$ & $21: 38$ & $21: 24$ & $21: 12$ & $21: 02$ & $20: 54$ & $20: 48$ \\
\hline 70. & $24: 41$ & $24: 24$ & $24: 08$ & $23: 54$ & $23: 42$ & $23: 32$ & $23: 24$ & $23: 16$ \\
\hline $80 \ldots \ldots$ & $27: 12$ & $26: 53$ & $26: 36$ & $26: 21$ & $26: 08$ & $25: 57$ & $25: 47$ & $25: 38$ \\
\hline 90. & $29: 33$ & $29: 14$ & $28: 57$ & $28: 41$ & $28: 29$ & $28: 17$ & $28: 07$ & $27: 58$ \\
\hline 100. & $31: 54$ & $31: 35$ & $31: 18$ & $31: 02$ & $30: 48$ & $30: 36$ & $30: 26$ & $30: 16$ \\
\hline $110 \ldots$ & $34: 12$ & $33: 53$ & $33: 35$ & $33: 19$ & $33: 05$ & $32: 52$ & $32: 41$ & $32: 31$ \\
\hline 120. & $36: 28$ & $36: 08$ & $35: 50$ & $35: 34$ & $35: 19$ & $35: 06$ & $34: 54$ & $34: 44$ \\
\hline 130. & $38: 30$ & $38: 10$ & $37: 52$ & $37: 35$ & $37: 20$ & $37: 06$ & $36: 53$ & $36: 42$ \\
\hline 140. & $40: 25$ & 40:05 & $39: 47$ & $39: 30$ & $39: 14$ & $39: 00$ & $38: 47$ & $38: 35$ \\
\hline 150. & $42: 20$ & $41: 59$ & $41: 40$ & $41: 22$ & $41: 06$ & $40: 52$ & $40: 38$ & $40: 26$ \\
\hline 160. & $44: 06$ & $43: 45$ & $43: 26$ & $43: 08$ & $42: 52$ & $42: 37$ & $42: 23$ & $42: 11$ \\
\hline 170. & $45: 50$ & $45: 30$ & $45: 11$ & $44: 53$ & $44: 36$ & $44: 21$ & 44:07 & $43: 54$ \\
\hline 180. & $47: 32$ & $47: 12$ & $46: 53$ & $46: 35$ & $46: 18$ & $46: 02$ & $45: 48$ & $45: 35$ \\
\hline
\end{tabular}

TABLE 19

Travel Times of sSS in Min.: Sec.

\begin{tabular}{|c|c|c|c|c|c|c|c|c|}
\hline \multirow{2}{*}{$\begin{array}{l}\text { Distance } \\
\text { in deg. }\end{array}$} & \multicolumn{8}{|c|}{ Depth of focus in $\mathrm{km}$. } \\
\hline & 100 & 200 & 300 & 400 & 500 & 600 & 700 & 800 \\
\hline 40. & $16: 58$ & $17: 12$ & $17: 22$ & & & & & \\
\hline 50. & $20: 04$ & $20: 19$ & $20: 32$ & $20: 42$ & & & & \\
\hline $60 .$. & $22: 47$ & $23: 04$ & $23: 18$ & $23: 31$ & $23: 35$ & & & \\
\hline $70 \ldots \ldots \ldots \ldots$ & $25: 20$ & $25: 37$ & $25: 52$ & $26: 06$ & $26: 18$ & $26: 27$ & $26: 30$ & \\
\hline $80 \ldots \ldots \ldots \ldots$ & $27: 50$ & $28: 07$ & $28: 24$ & $28: 39$ & $28: 52$ & $29: 03$ & $29: 11$ & $29: 17$ \\
\hline 90. & $30: 18$ & $30: 37$ & $30: 53$ & $31: 08$ & $31: 21$ & $31: 32$ & $31: 41$ & $31: 48$ \\
\hline 100. & $32: 39$ & $32: 57$ & $33: 14$ & $33: 30$ & $33: 43$ & $33: 55$ & $34: 05$ & $34: 14$ \\
\hline 110. & $34: 57$ & $35: 15$ & $35: 32$ & $35: 48$ & $36: 02$ & $36: 14$ & $36: 24$ & $26: 34$ \\
\hline 120. & $37: 13$ & $37: 31$ & $37: 49$ & $38: 06$ & $38: 20$ & $38: 33$ & $38: 44$ & $38: 54$ \\
\hline $130 \ldots$ & $39: 14$ & $39: 33$ & $39: 52$ & $40: 09$ & $40: 24$ & $40: 38$ & $40: 50$ & $41: 01$ \\
\hline 140. & $41: 10$ & $41: 29$ & $41: 47$ & $42: 04$ & $42: 19$ & $42: 33$ & $42: 46$ & $42: 57$ \\
\hline 150. & $43: 05$ & $43: 24$ & $43: 42$ & $43: 59$ & $44: 14$ & $44: 29$ & $44: 42$ & $44: 53$ \\
\hline 160. & $44: 51$ & $45: 11$ & $45: 30$ & $45: 48$ & $46: 04$ & $46: 19$ & $46: 33$ & $46: 45$ \\
\hline $170 \ldots \ldots$ & $46: 35$ & $46: 55$ & $47: 14$ & $47: 32$ & $47: 49$ & $48: 04$ & $48: 18$ & $48: 31$ \\
\hline $180 \ldots \ldots \ldots \ldots$ & $48: 16$ & $48: 36$ & $48: 55$ & $49: 13$ & $49: 30$ & $49: 45$ & $49: 59$ & $50: 12$ \\
\hline
\end{tabular}


TABLE 20

Travel Times of SSS in Min.: Sec.

\begin{tabular}{|c|c|c|c|c|c|c|c|c|}
\hline \multirow{2}{*}{$\begin{array}{l}\text { Distance } \\
\text { in deg. }\end{array}$} & \multicolumn{8}{|c|}{ Depth of focus in $\mathrm{km}$. } \\
\hline & 100 & 200 & 300 & 400 & 500 & 600 & 700 & 800 \\
\hline 50. & $21: 17$ & 21:06 & $20: 58$ & $20: 53$ & & & & \\
\hline 60 . & $24: 58$ & $24: 35$ & $24: 24$ & $24: 15$ & $24: 10$ & & & \\
\hline 70 . & $27: 56$ & $27: 41$ & $27: 27$ & $27: 16$ & $27: 06$ & $27: 00$ & $26: 58$ & \\
\hline 80. & $30: 48$ & $30: 31$ & $30: 16$ & $30: 03$ & 29:51 & $29: 42$ & $29: 35$ & $29: 30$ \\
\hline 90. & $33: 25$ & $33: 08$ & $32: 52$ & $32: 38$ & $32: 26$ & $32: 16$ & $32: 07$ & $32: 02$ \\
\hline 100. & $35: 58$ & $35: 41$ & $35: 25$ & $35: 11$ & $34: 59$ & $34: 49$ & $34: 39$ & $34: 32$ \\
\hline 110 . & $38: 30$ & $38: 13$ & $37: 57$ & $37: 42$ & $37: 29$ & $37: 18$ & $37: 08$ & $37: 01$ \\
\hline 120. & $40: 57$ & $40: 40$ & $40: 23$ & $40: 08$ & $39: 55$ & $39: 44$ & $39: 34$ & $39: 26$ \\
\hline 130. & $43: 22$ & $43: 04$ & $42: 47$ & $42: 32$ & $42: 19$ & $42: 07$ & $41: 56$ & $41: 48$ \\
\hline 140 . & $45: 46$ & $45: 28$ & 45:11 & $44: 56$ & $44: 43$ & $44: 30$ & $44: 18$ & $44: 10$ \\
\hline 150. & $48: 07$ & $47: 49$ & $47: 32$ & $47: 16$ & $47: 02$ & $46: 49$ & $46: 37$ & $46: 28$ \\
\hline 160 . & $50: 23$ & $50: 05$ & $49: 47$ & $49: 31$ & $49: 17$ & $49: 04$ & $48: 52$ & $48: 43$ \\
\hline 170 & $52: 39$ & $52: 21$ & $52: 03$ & $51: 46$ & $51: 32$ & $51: 19$ & $51: 07$ & $50: 57$ \\
\hline 180. & $54: 55$ & $54: 36$ & $54: 18$ & $54: 01$ & $53: 47$ & $53: 34$ & $53: 22$ & $53: 12$ \\
\hline
\end{tabular}

TABLE 21

Travel Times of SP in Min.: Sec.

\begin{tabular}{|c|c|c|c|c|c|c|c|c|}
\hline \multirow{2}{*}{$\begin{array}{l}\text { Distance } \\
\text { in deg. }\end{array}$} & \multicolumn{8}{|c|}{ Depth of focus in $\mathrm{km}$. } \\
\hline & 100 & 200 & 300 & 400 & 500 & 600 & 700 & 800 \\
\hline 70. & $20: 35$ & $20: 16$ & $19: 59$ & $19: 43$ & $19: 28$ & $19: 15$ & 19:03 & $18: 53$ \\
\hline 75 & $21: 40$ & $21: 21$ & $21: 03$ & $20: 46$ & $20: 30$ & $20: 16$ & $20: 04$ & $19: 53$ \\
\hline 80. & $22: 43$ & $22: 23$ & $22: 04$ & $21: 47$ & $21: 31$ & $21: 16$ & $21: 03$ & $20: 52$ \\
\hline 85 . & $23: 42$ & $23: 22$ & $23: 03$ & $22: 46$ & $22: 30$ & $22: 15$ & $22: 02$ & $21: 50$ \\
\hline $90 \ldots$ & $24: 38$ & $24: 18$ & $23: 59$ & $23: 42$ & $23: 27$ & $23: 13$ & $22: 59$ & $22: 47$ \\
\hline 95 . & $25: 34$ & $25: 14$ & $24: 55$ & $24: 37$ & $24: 21$ & $24: 07$ & $23: 53$ & $23: 41$ \\
\hline 100 . & $26: 29$ & $26: 08$ & $25: 49$ & $25: 31$ & $25: 15$ & $25: 00$ & $24: 46$ & $24: 34$ \\
\hline 105 & $27: 21$ & $27: 01$ & $26: 42$ & $26: 24$ & $26: 08$ & $25: 53$ & $25: 39$ & $25: 27$ \\
\hline 110 & $28: 13$ & $27: 53$ & $27: 33$ & $27: 15$ & $26: 59$ & $26: 44$ & $26: 30$ & $26: 17$ \\
\hline 115 & $29: 04$ & $28: 44$ & $28: 24$ & $28: 05$ & $27: 49$ & $27: 33$ & $27: 19$ & $27: 06$ \\
\hline 120 & $29: 51$ & $29: 31$ & $29: 11$ & $28: 52$ & $28: 36$ & $28: 20$ & $28: 05$ & $27: 52$ \\
\hline 125 & $30: 37$ & $30: 17$ & $29: 57$ & $29: 38$ & $29: 21$ & $29: 05$ & $28: 50$ & $28: 37$ \\
\hline 130. & $31: 20$ & $31: 00$ & $30: 40$ & $30: 21$ & $30: 04$ & $29: 48$ & $29: 33$ & $29: 20$ \\
\hline 135. & $32: 02$ & $31: 41$ & $31: 21$ & $31: 03$ & $30: 46$ & $30: 30$ & $30: 15$ & $30: 02$ \\
\hline 140 & $32: 44$ & $32: 23$ & $32: 03$ & $31: 44$ & $31: 27$ & $31: 11$ & $30: 56$ & $30: 43$ \\
\hline
\end{tabular}


$\mathrm{PKKP}-\mathrm{P}$. The intervals $p \mathrm{P}^{\prime} \mathrm{P}^{\prime}-\mathrm{P}^{\prime} \mathrm{P}^{\prime}$ and $s \mathrm{P}^{\prime} \mathrm{P}^{\prime}-\mathrm{P}^{\prime} \mathrm{P}^{\prime}$ are about the same as $\mathrm{pP}^{\prime}-\mathrm{P}^{\prime}$ and $\mathrm{s}^{\prime}-\mathrm{P}^{\prime}$ for equal hypocentral depth, as tabulated in tables 8 and 9 .

Similar remarks apply to $\mathrm{P}^{\prime} \mathrm{P}^{\prime} \mathrm{P}^{\prime}$ (table 36 ) and $\mathrm{SKPP}^{\prime}$ (table 37 ). With respect to the latter the effect of hypocentral depth on the interval SKPP' $-\mathrm{P}$ is larger than for $\mathbf{P}^{\prime} \mathrm{P}^{\prime}-\mathrm{P}$. Intervals $\mathrm{PKSP}^{\prime}-\mathrm{SKPP}^{\prime}$ are given in the last line of table 37.

TABLE 22

Travel Times of PS in Min.: Sec.

\begin{tabular}{|c|c|c|c|c|c|c|c|c|}
\hline \multirow{2}{*}{$\begin{array}{l}\text { Distance } \\
\text { in deg. }\end{array}$} & \multicolumn{8}{|c|}{ Depth of focus in $\mathrm{km}$. } \\
\hline & 100 & 200 & 300 & 400 & 500 & 600 & 700 & 800 \\
\hline $70 \ldots \ldots$ & $20: 51$ & $20: 47$ & & & & & & \\
\hline 75 & $21: 53$ & $21: 48$ & & & & & & \\
\hline 80. & $22: 54$ & $22: 48$ & $22: 43$ & & & & & \\
\hline 85. & $23: 54$ & $23: 47$ & $23: 41$ & $23: 37$ & & & & \\
\hline 90 . & $24: 50$ & $24: 43$ & $24: 37$ & $24: 33$ & $24: 30$ & & & \\
\hline 95 . & $25: 46$ & $25: 39$ & $25: 33$ & $25: 28$ & $25: 25$ & $25: 23$ & & \\
\hline $100 \ldots \ldots$ & $26: 42$ & $26: 35$ & $26: 28$ & $26: 23$ & $26: 19$ & $26: 17$ & & \\
\hline $105 \ldots \ldots$ & $27: 36$ & $27: 29$ & $37: 21$ & $27: 15$ & $27: 11$ & $27: 09$ & $27: 08$ & \\
\hline $110 \ldots$ & $28: 28$ & $28: 20$ & $28: 12$ & $28: 06$ & $28: 02$ & $27: 59$ & $27: 57$ & $27: 55$ \\
\hline 115. & $29: 17$ & $29: 08$ & $29: 00$ & $28: 54$ & $28: 48$ & $28: 44$ & $28: 41$ & $28: 38$ \\
\hline 120 . & $30: 04$ & $29: 55$ & $29: 47$ & $29: 39$ & $29: 32$ & $29: 27$ & $29: 24$ & $29: 20$ \\
\hline 125. & $30: 48$ & $30: 39$ & $30: 30$ & $30: 22$ & $30: 15$ & $30: 10$ & $30: 06$ & $30: 02$ \\
\hline 130. & $31: 31$ & $31: 21$ & $31: 12$ & $31: 04$ & $30: 57$ & $30: 52$ & $30: 48$ & $30: 44$ \\
\hline 135. & $32: 13$ & $32: 04$ & $31: 54$ & $31: 46$ & $31: 39$ & $31: 34$ & $31: 30$ & $31: 26$ \\
\hline $140 \ldots$ & $32: 55$ & $32: 45$ & $32: 36$ & $32: 28$ & $32: 21$ & $32: 16$ & $32: 12$ & $32: 08$ \\
\hline
\end{tabular}

TABLE 23

Travel Times of pPS or pSP in Mrn.: Sec.

\begin{tabular}{|c|c|c|c|c|c|c|c|c|}
\hline \multirow{2}{*}{$\begin{array}{l}\text { Distance } \\
\text { in deg. }\end{array}$} & \multicolumn{8}{|c|}{ Depth of focus in $\mathrm{km}$. } \\
\hline & 100 & 200 & 300 & 400 & 500 & 600 & 700 & 800 \\
\hline $90 \ldots \ldots$ & 25:06 & $25: 13$ & & & & & & \\
\hline $100 \ldots \ldots$ & $26: 57$ & $27: 05$ & $27: 08$ & & & & & \\
\hline $110 \ldots \ldots$ & $28: 46$ & $28: 55$ & $29: 01$ & $29: 06$ & & & & \\
\hline $120 \ldots \ldots \ldots \ldots$ & $30: 23$ & $30: 32$ & $30: 39$ & $30: 45$ & $30: 48$ & & & \\
\hline 130 . & $31: 52$ & $32: 02$ & $32: 10$ & $32: 18$ & $32: 22$ & $32: 26$ & & \\
\hline 140 . & $33: 18$ & $33: 28$ & $33: 36$ & $33: 44$ & $33: 49$ & $33: 54$ & $33: 58$ & \\
\hline 150. & $34: 43$ & $34: 53$ & $35: 02$ & $35: 10$ & $35: 16$ & $35: 22$ & $35: 28$ & $35: 30$ \\
\hline 160. & $36: 08$ & $36: 18$ & $36: 27$ & $36: 35$ & $36: 41$ & $36: 46$ & $36: 52$ & $36: 55$ \\
\hline
\end{tabular}


TABLE 24

Travel Times of sPS or sSP in Min.: Sec.

\begin{tabular}{|c|c|c|c|c|c|c|c|c|}
\hline \multirow{2}{*}{$\begin{array}{l}\text { Distance } \\
\text { in deg. }\end{array}$} & \multicolumn{8}{|c|}{ Depth of focus in $\mathrm{km}$. } \\
\hline & 100 & 200 & 300 & 400 & 500 & 600 & 700 & 800 \\
\hline 70 & $21: 23$ & $21: 41$ & $21: 59$ & $22: 16$ & $22: 31$ & $22: 44$ & $22: 55$ & $23: 05$ \\
\hline 80 & $23: 23$ & $23: 42$ & $24: 00$ & $24: 17$ & $24: 32$ & $24: 46$ & $24: 58$ & $25: 08$ \\
\hline 90 . & $25: 20$ & $25: 40$ & $25: 58$ & $26: 16$ & $26: 32$ & $26: 46$ & $26: 59$ & $27: 10$ \\
\hline 100 . & $27: 11$ & $27: 31$ & $27: 49$ & $28: 07$ & $28: 23$ & $28: 38$ & $28: 52$ & $29: 04$ \\
\hline $110 \ldots$. & $28: 58$ & $29: 18$ & $29: 36$ & $29: 54$ & $30: 10$ & $30: 26$ & $30: 40$ & $30: 53$ \\
\hline 120. & $30: 36$ & $30: 57$ & $31: 16$ & $31: 34$ & $31: 50$ & $32: 06$ & $32: 20$ & $32: 35$ \\
\hline 130 . & $32: 06$ & $32: 27$ & $32: 47$ & $33: 05$ & $33: 22$ & $33: 38$ & $33: 52$ & $34: 06$ \\
\hline 140 . & $33: 31$ & $33: 52$ & $34: 12$ & $34: 31$ & $34: 48$ & $35: 04$ & $35: 19$ & $35: 33$ \\
\hline 150. & $34: 56$ & $35: 17$ & $35: 37$ & $35: 56$ & $36: 13$ & $36: 30$ & $36: 45$ & $36: 59$ \\
\hline 160. & $36: 21$ & $36: 41$ & $37: 02$ & $37: 21$ & $37: 38$ & $37: 55$ & $38: 10$ & $38: 24$ \\
\hline
\end{tabular}

TABLE 25

Travel Times of SPP in Min.: Sec.

\begin{tabular}{|c|c|c|c|c|c|c|c|c|}
\hline \multirow{2}{*}{$\begin{array}{l}\text { Distance } \\
\text { in deg. }\end{array}$} & \multicolumn{8}{|c|}{ Depth of focus in $\mathrm{km}$. } \\
\hline & 100 & 200 & 300 & 400 & 500 & 600 & 700 & 800 \\
\hline 100. & $27: 24$ & $27: 04$ & $26: 45$ & $26: 28$ & $26: 12$ & 25:57 & $25: 44$ & $25: 32$ \\
\hline 110. & $29: 15$ & $28: 55$ & $28: 36$ & $28: 19$ & $28: 03$ & $27: 48$ & $27: 35$ & $27: 23$ \\
\hline 120. & 31:04 & $30: 44$ & $30: 25$ & 30:07 & $29: 51$ & $29: 36$ & $29: 22$ & $29: 10$ \\
\hline 130. & $32: 50$ & $32: 30$ & $32: 11$ & $31: 53$ & $31: 37$ & $31: 22$ & $31: 08$ & $30: 55$ \\
\hline 140. & $34: 30$ & $34: 10$ & $33: 51$ & $33: 33$ & $33: 17$ & $33: 02$ & $32: 48$ & $32: 34$ \\
\hline 150. & $36: 03$ & $35: 43$ & $35: 24$ & $35: 07$ & 34:50 & $34: 34$ & $34: 20$ & $34: 06$ \\
\hline 160. & $37: 35$ & $37: 14$ & $36: 55$ & $36: 38$ & $36: 21$ & $36: 05$ & $35: 50$ & $35: 36$ \\
\hline 170. & $39: 06$ & $38: 45$ & $38: 25$ & $38: 08$ & 37:51 & $37: 35$ & $37: 20$ & $37: 06$ \\
\hline
\end{tabular}

TABLE 26

Traver Times of PPS or PSP in Min.: Sec.

\begin{tabular}{|c|c|c|c|c|c|c|c|c|}
\hline \multirow{2}{*}{$\begin{array}{l}\text { Distance } \\
\text { in deg. }\end{array}$} & \multicolumn{8}{|c|}{ Depth of focus in $\mathrm{km}$. } \\
\hline & 100 & 200 & 300 & 400 & 500 & 600 & 700 & 800 \\
\hline 100. & $27: 35$ & $27: 28$ & $27: 24$ & $27: 23$ & & & & \\
\hline 110. & $29: 27$ & $29: 20$ & $29: 14$ & 29:12 & $29: 11$ & & & \\
\hline 120. & $31: 17$ & 31:09 & 31:02 & $30: 58$ & $30: 55$ & & & \\
\hline 130. & $33: 05$ & $32: 57$ & $32: 49$ & $32: 43$ & $32: 38$ & $32: 36$ & & \\
\hline 140. & $34: 48$ & $34: 39$ & $34: 31$ & $34: 25$ & $34: 20$ & $34: 16$ & 34:14 & \\
\hline 150. & $36: 20$ & $36: 11$ & $36: 03$ & $35: 56$ & $35: 51$ & $35: 47$ & $35: 44$ & $35: 43$ \\
\hline 160. & $37: 52$ & $37: 43$ & $37: 34$ & $37: 27$ & $37: 21$ & $37: 17$ & $37: 14$ & $37: 13$ \\
\hline 170. & $39: 23$ & $39: 14$ & $39: 05$ & $38: 58$ & $38: 52$ & $38: 47$ & $38: 44$ & $38: 43$ \\
\hline
\end{tabular}


Times for the simpler waves reflected from the outside of the core are tabulated in tables 38 to 44 .

Theoretically, the travel time of the $\mathrm{G}$ wave should be the same for all depths of focus. Very long-period waves of this type may be observable in deep-focus shocks.

The data given in tables 6 to 44 can be used to construct travel-time curves for the various tabulated depths. As an example, the curves for a depth of 400 $\mathrm{km}$. are given in figure 1 (the folded graph at the end of this paper). With increasing depth, the number of observable phases theoretically should decrease, as the critical distances, which are the lower limits for pairs of phases such as $\mathrm{pP}$ and $\mathrm{PP}, \mathrm{sP}$ and $\mathrm{SP}$, increase with increasing hypocentral depth. For waves

TABLE 27

Travel Times of SKP, PKS, pPKS or pSKP, and sPKS or sSKP, in Min.: Sec.

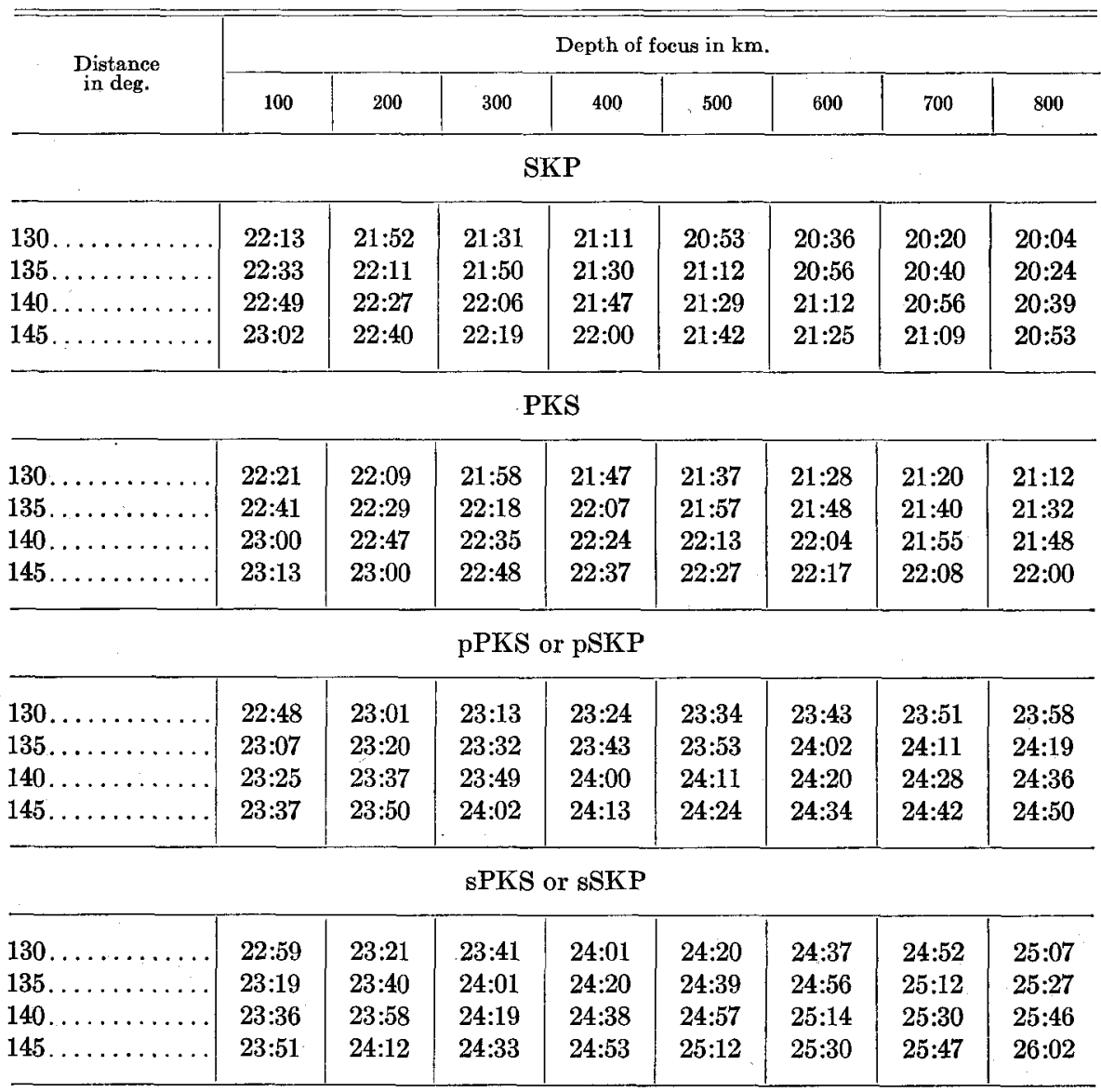


TABLE 28

Travel Times of SKS in Min.: Sec.

\begin{tabular}{|c|c|c|c|c|c|c|c|c|}
\hline \multirow{2}{*}{$\begin{array}{l}\text { Distance } \\
\text { in deg. }\end{array}$} & & \multicolumn{5}{|c|}{ epth of focus in km. } \\
\hline & 100 & 200 & 300 & 400 & 500 & 600 & 700 & 800 \\
\hline 80 & $22: 07$ & $21: 45$ & $21: 26$ & $21: 08$ & $20: 51$ & $20: 36$ & $20: 22$ & $20: 10$ \\
\hline 85. & $22: 39$ & $22: 17$ & $21: 58$ & $21: 40$ & $21: 23$ & $21: 07$ & $20: 53$ & $20: 41$ \\
\hline 90 . & 23:09 & $22: 47$ & $22: 27$ & $22: 09$ & $21: 52$ & $21: 37$ & $21: 23$ & $21: 10$ \\
\hline 95 . & $23: 37$ & $23: 16$ & $22: 56$ & $22: 37$ & $22: 20$ & $22: 05$ & $21: 50$ & $21: 37$ \\
\hline $100 \ldots$ & $24: 03$ & $23: 42$ & $23: 23$ & $23: 03$ & $22: 46$ & $22: 30$ & $22: 15$ & $22: 02$ \\
\hline 105 . & $24: 28$ & 24:07 & $23: 47$ & $23: 28$ & $23: 11$ & $22: 54$ & $22: 38$ & $22: 24$ \\
\hline 110 . & $24: 52$ & $24: 30$ & $24: 10$ & $23: 51$ & $23: 33$ & $23: 16$ & $23: 00$ & $22: 45$ \\
\hline $115 \ldots \ldots \ldots \ldots$ & $25: 14$ & $24: 52$ & $24: 32$ & $24: 13$ & $23: 54$ & $23: 37$ & $23: 21$ & $23: 06$ \\
\hline $120 \ldots \ldots \ldots \ldots$ & $25: 34$ & $25: 13$ & $24: 53$ & $24: 33$ & $24: 14$ & $23: 57$ & $23: 41$ & $23: 25$ \\
\hline $125 \ldots \ldots \ldots$ & $25: 49$ & $25: 28$ & 25:08 & $24: 48$ & $24: 30$ & $24: 13$ & $23: 56$ & $23: 40$ \\
\hline 130. & $26: 01$ & $25: 40$ & $25: 20$ & $25: 00$ & $24: 42$ & $24: 24$ & $24: 07$ & $23: 51$ \\
\hline 135. & $26: 12$ & $25: 51$ & $25: 31$ & $25: 11$ & $24: 52$ & $24: 34$ & $24: 17$ & $24: 01$ \\
\hline 140 . & $26: 22$ & $26: 00$ & $25: 40$ & $25: 20$ & $25: 01$ & $24: 43$ & $24: 26$ & $24: 09$ \\
\hline 145 & $26: 31$ & $26: 08$ & $25: 47$ & $25: 28$ & $25: 09$ & $24: 50$ & $24: 33$ & $24: 16$ \\
\hline 150 . & $26: 36$ & $26: 14$ & $25: 53$ & $25: 33$ & $25: 14$ & $24: 56$ & $24: 38$ & $24: 21$ \\
\hline 180. & $26: 51$ & $26: 28$ & $26: 07$ & $25: 47$ & $25: 27$ & $25: 09$ & $24: 51$ & $24: 34$ \\
\hline
\end{tabular}

TABLE 29

Differences in Travel Times, pSKS-SKS and sSKS-SKS, in Min.: Sec.

\begin{tabular}{|c|c|c|c|c|c|c|c|c|}
\hline \multirow{2}{*}{$\begin{array}{l}\text { Distance } \\
\text { in deg. }\end{array}$} & \multicolumn{8}{|c|}{ Depth of focus in $\mathrm{km}$. } \\
\hline & 100 & 200 & 300 & 400 & 500 & 600 & 700 & 800 \\
\hline \multicolumn{9}{|c|}{ pSKS-SKS } \\
\hline 110. & $0: 37$ & $1: 09$ & $1: 38$ & $2: 06$ & $2: 32$ & $2: 56$ & $3: 16$ & $3: 33$ \\
\hline 180 & $0: 39$ & $1: 14$ & $1: 47$ & $2: 18$ & $2: 48$ & $3: 16$ & $3: 44$ & $4: 11$ \\
\hline \multicolumn{9}{|c|}{ sSKS-SKS } \\
\hline 110. & $0: 48$ & $1: 31$ & $2: 11$ & $2: 50$ & $3: 27$ & $4: 01$ & $4: 33$ & $5: 03$ \\
\hline 180. & $0: 48$ & $1: 35$ & $2: 16$ & $2: 56$ & $3: 36$ & $4: 12$ & $4: 48$ & $5: 22$ \\
\hline
\end{tabular}


TABLE 30

Travel Times of SKKS in Min.: Sec.

\begin{tabular}{r|c|c|c|c|c|c|c|c}
\hline \hline \multirow{2}{*}{$\begin{array}{c}\text { Distance } \\
\text { in deg. }\end{array}$} & \multicolumn{9}{|c|}{ Depth of focus in km. } \\
\cline { 2 - 8 } & 100 & 200 & 300 & 400 & 500 & 600 & 700 & 800 \\
\hline $90 \ldots \ldots \ldots \ldots \ldots$ & $23: 17$ & $22: 55$ & $22: 35$ & $22: 16$ & $21: 59$ & $21: 42$ & $21: 26$ & $21: 12$ \\
$100 \ldots \ldots \ldots \ldots \ldots$ & $24: 24$ & $24: 02$ & $23: 42$ & $23: 23$ & $23: 05$ & $22: 48$ & $22: 32$ & $22: 17$ \\
$110 \ldots \ldots \ldots \ldots \ldots$ & $25: 28$ & $25: 06$ & $24: 46$ & $24: 27$ & $24: 09$ & $23: 52$ & $23: 36$ & $23: 21$ \\
$120 \ldots \ldots \ldots \ldots \ldots$ & $26: 29$ & $26: 07$ & $25: 46$ & $25: 27$ & $25: 09$ & $24: 52$ & $24: 36$ & $24: 21$ \\
& & & & & & & & \\
$130 \ldots \ldots \ldots \ldots$ & $27: 28$ & $27: 06$ & $26: 45$ & $26: 26$ & $26: 08$ & $25: 51$ & $25: 35$ & $25: 20$ \\
$140 \ldots \ldots \ldots \ldots$ & $28: 27$ & $28: 05$ & $27: 44$ & $27: 25$ & $27: 07$ & $26: 50$ & $26: 34$ & $26: 19$ \\
$150 \ldots \ldots \ldots \ldots$ & $29: 24$ & $29: 02$ & $28: 42$ & $28: 23$ & $28: 05$ & $27: 47$ & $27: 31$ & $27: 16$ \\
$160 \ldots \ldots \ldots \ldots \ldots$ & $30: 21$ & $30: 00$ & $29: 39$ & $29: 20$ & $29: 02$ & $28: 44$ & $28: 28$ & $28: 13$ \\
\hline
\end{tabular}

TABLE 31

Travet Times of SKSP in Min.: Sec

\begin{tabular}{|c|c|c|c|c|c|c|c|c|}
\hline \multirow{2}{*}{$\begin{array}{l}\text { Distance } \\
\text { in deg. }\end{array}$} & \multicolumn{8}{|c|}{ Depth of focus in $\mathrm{km}$. } \\
\hline & 100 & 200 & 300 & 400 & 500 & 600 & 700 & 800 \\
\hline 120. & $30: 06$ & $29: 45$ & $29: 24$ & $29: 05$ & $28: 47$ & $28: 31$ & $28: 16$ & $28: 01$ \\
\hline 130. & $31: 07$ & $30: 46$ & $30: 26$ & $30: 06$ & $29: 48$ & $29: 32$ & $29: 17$ & $29: 02$ \\
\hline $140 \ldots$ & $32: 08$ & $31: 46$ & $31: 26$ & $31: 07$ & $30: 49$ & $30: 33$ & $30: 17$ & $30: 02$ \\
\hline 150. & $33: 08$ & $32: 46$ & $32: 26$ & $32: 07$ & $31: 50$ & $31: 33$ & $31: 17$ & $31: 02$ \\
\hline 160. & $34: 09$ & $33: 47$ & $33: 26$ & $33: 07$ & $32: 49$ & $32: 33$ & $32: 17$ & $32: 02$ \\
\hline 170. & $35: 08$ & $34: 47$ & $34: 26$ & $34: 06$ & $33: 49$ & $33: 32$ & $33: 16$ & $33: 01$ \\
\hline
\end{tabular}

TABLE 32

Travel Times of PSKS in Min.: Sec.

\begin{tabular}{l|c|c|c|c|c|c|c|c}
\hline \hline \multirow{2}{*}{$\begin{array}{c}\text { Distance } \\
\text { in deg. }\end{array}$} & \multicolumn{2}{|c|}{ Depth of focus in km. } \\
\cline { 2 - 9 } & 100 & 200 & 300 & 400 & 500 & 600 & 700 & 800 \\
\hline $120 \ldots \ldots \ldots \ldots \ldots$ & $30: 19$ & $30: 08$ & $29: 57$ & $29: 47$ & $29: 38$ & $29: 30$ & $29: 23$ & $29: 17$ \\
$130 \ldots \ldots \ldots \ldots$ & $31: 20$ & $31: 09$ & $30: 58$ & $30: 48$ & $30: 39$ & $30: 31$ & $30: 24$ & $30: 18$ \\
$140 \ldots \ldots \ldots \ldots \ldots$ & $32: 20$ & $32: 09$ & $31: 59$ & $31: 49$ & $31: 40$ & $31: 32$ & $31: 25$ & $31: 18$ \\
& & & & & & & & \\
$150 \ldots \ldots \ldots \ldots$ & $33: 20$ & $33: 08$ & $32: 58$ & $32: 48$ & $32: 39$ & $32: 31$ & $32: 25$ & $32: 18$ \\
$160 \ldots \ldots \ldots \ldots$ & $34: 19$ & $34: 08$ & $33: 57$ & $33: 47$ & $33: 38$ & $33: 30$ & $33: 24$ & $33: 17$ \\
$170 \ldots \ldots \ldots \ldots$ & $35: 18$ & $35: 07$ & $34: 56$ & $34: 46$ & $34: 37$ & $34: 29$ & $34: 22$ & $34: 16$ \\
\hline
\end{tabular}


TABLE 33

Differences in Travel Trmes, PKKP-P, in Min.:Segc. (the Valdes Are the Same Within the Limits of Error for Depths Up to 800 KM.) and TrAvel Times of PKKP for Various Depths as a Function of the Distance $\Delta$, in Degrees

\begin{tabular}{|c|c|c|c|c|c|c|c|c|c|c|}
\hline \multirow{2}{*}{$\Delta$} & \multirow{2}{*}{ PKKP-P } & \multicolumn{9}{|c|}{ Travel time of PKKP for various depths in $\mathrm{km}$. } \\
\hline & & 25 & 100 & 200 & 300 & 400 & 500 & 600 & 700 & 800 \\
\hline 95 & 17:03 & $30: 30$ & $30: 19$ & $30: 07$ & $29: 55$ & $29: 45$ & $29: 36$ & $29: 27$ & $29: 18$ & $29: 09$ \\
\hline 96 & $16: 55$ & $30: 27$ & $30: 16$ & $30: 03$ & $29: 52$ & $29: 42$ & $29: 33$ & $29: 23$ & $29: 14$ & $29: 06$ \\
\hline 97 & $16: 48$ & $: 24$ & $: 13$ & $30: 00$ & $: 49$ & $: 39$ & $: 30$ & $: 20$ & :11 & $29: 03$ \\
\hline 98 & $: 40$ & :22 & $: 10$ & $29: 57$ & $: 46$ & :36 & :27 & :17 & $: 08$ & $29: 00$ \\
\hline 99 & :33 & :19 & $: 07$ & $: 54$ & $: 43$ & $: 33$ & $: 24$ & :14 & $: 05$ & $28: 57$ \\
\hline 100 & :25 & $: 16$ & $: 04$ & $: 51$ & :39 & :30 & :21 & :11 & $29: 02$ & $: 54$ \\
\hline 101 & :18 & :13 & $30: 01$ & $: 48$ & :36 & :26 & :18 & :08 & $28: 59$ & $: 51$ \\
\hline 102 & :10 & $: 09$ & $29: 57$ & :45 & :33 & :23 & :15 & $: 05$ & $: 56$ & :48 \\
\hline 103 & $16: 03$ & $: 06$ & $: 54$ & $: 42$ & :30 & :20 & :12 & $29: 02$ & $: 53$ & $: 45$ \\
\hline 104 & $15: 55$ & :03 & $: 51$ & :39 & $: 27$ & :17 & :08 & $28: 59$ & $: 50$ & $: 42$ \\
\hline 105 & $: 48$ & $30: 00$ & $: 48$ & :36 & $: 24$ & :14 & $: 05$ & $: 56$ & $: 47$ & :39 \\
\hline 106 & $: 40$ & $29: 56$ & $: 45$ & :33 & $: 21$ & :11 & $29: 02$ & :53 & $: 44$ & :36 \\
\hline 107 & :33 & $: 53$ & :42 & :30 & :18 & $: 08$ & $28: 59$ & $: 49$ & $: 41$ & $: 33$ \\
\hline 108 & $: 25$ & $: 50$ & :38 & :26 & $: 14$ & $: 05$ & $: 56$ & $: 46$ & $: 38$ & :30 \\
\hline 109 & :18 & $: 46$ & :35 & :23 & $: 11$ & $29: 01$ & $: 52$ & $: 43$ & $: 35$ & $: 27$ \\
\hline 110 & :10 & $: 43$ & :32 & $: 20$ & :08 & $28: 57$ & $: 49$ & :39 & :31 & :24 \\
\hline 111 & 15:03 & $: 40$ & :29 & $: 17$ & :04 & $: 54$ & $: 45$ & :36 & $: 28$ & :21 \\
\hline 112 & $14: 55$ & $: 37$ & $: 25$ & :13 & $29: 01$ & $: 51$ & $: 42$ & :33 & :24 & 17 \\
\hline 113 & $: 47$ & :33 & :22 & :10 & $28: 58$ & $: 47$ & $: 38$ & $: 29$ & :21 & :14 \\
\hline 114 & :39 & :30 & :18 & $: 07$ & :55 & $: 44$ & :35 & :26 & :17 & :10 \\
\hline 115 & :30 & $: 26$ & :15 & $29: 03$ & :51 & $: 40$ & :31 & :22 & :13 & :07 \\
\hline 116 & $: 21$ & :22 & :11 & $28: 59$ & :48 & :37 & $: 28$ & $: 19$ & :10 & $28: 03$ \\
\hline 118 & $14: 05$ & $29: 13$ & $29: 02$ & $: 51$ & :39 & :28 & :19 & :10 & $28: 01$ & $27: 54$ \\
\hline 120 & $13: 47$ & $29: 03$ & $28: 53$ & $: 41$ & :29 & $: 19$ & :10 & $28: 01$ & $27: 52$ & $: 45$ \\
\hline 122 & $13: 25$ & $28: 50$ & $: 40$ & :28 & :17 & 28:06 & $27: 58$ & $27: 49$ & $: 41$ & $: 35$ \\
\hline 124 & $(13: 02)$ & $(28: 40)$ & $(28: 29)$ & $(28: 16)$ & $(28: 05)$ & $27: 53$ & $: 44$ & $: 35$ & $: 26$ & :20 \\
\hline
\end{tabular}

TABLE 34

Travel Times of SKKP in Mrn.:Sec.

\begin{tabular}{l|c|c|c|c|c|c|c|c}
\hline \multirow{2}{*}{$\begin{array}{c}\text { Distance } \\
\text { in deg. }\end{array}$} & \multicolumn{7}{|c|}{ Depth of focus in km. } \\
\cline { 2 - 9 } & $\mathbf{1 0 0}$ & 200 & 300 & 400 & 500 & 600 & 700 & 800 \\
\hline $120 \ldots \ldots \ldots \ldots \ldots$ & $32: 40$ & $32: 18$ & $31: 56$ & $31: 35$ & $31: 15$ & $30: 56$ & $30: 39$ & $30: 22$ \\
$125 \ldots \ldots \ldots \ldots \ldots$ & $32: 25$ & $32: 03$ & $31: 41$ & $31: 20$ & $31: 00$ & $30: 41$ & $30: 24$ & $30: 07$ \\
$130 \ldots \ldots \ldots \ldots \ldots$ & $32: 08$ & $31: 46$ & $31: 24$ & $31: 03$ & $30: 43$ & $30: 25$ & $30: 08$ & $29: 51$ \\
& & & & & & & & \\
$135 \ldots \ldots \ldots \ldots \ldots$ & $31: 50$ & $31: 28$ & $31: 06$ & $30: 45$ & $30: 25$ & $30: 07$ & $29: 50$ & $29: 34$ \\
$140 \ldots \ldots \ldots \ldots$ & $31: 30$ & $31: 08$ & $30: 46$ & $30: 25$ & $30: 06$ & $29: 48$ & $29: 31$ & $29: 15$ \\
$\mathbf{1 4 5} \ldots \ldots \ldots \ldots \ldots$ & $31: 08$ & $30: 46$ & $30: 25$ & $30: 04$ & $29: 45$ & $29: 27$ & $29: 10$ & $28: 54$ \\
\hline
\end{tabular}


TABLE 35

Differences in Travel Times, $P^{\prime} P^{\prime}-P$, and Travel Tmes of $P^{\prime} P^{\prime}$ in Min.:Sec. for Various Depths of Focus in KM. and Distances $\Delta$ in Degrees (The data below the figure with the asterisk concern the diffracted $\mathbf{P}^{\prime} \mathrm{P}^{\prime}$.)

\begin{tabular}{|c|c|c|c|c|c|c|c|c|c|c|}
\hline \multirow{2}{*}{$\Delta$} & \multicolumn{5}{|c|}{$P^{\prime} P^{\prime}-P$ for depth of focus } & \multicolumn{5}{|c|}{$P^{\prime} P^{\prime}-O$ for depth of focus } \\
\hline & $\mathbf{0}$ & 200 & 400 & 600 & 800 & 0 & 200 & 400 & 600 & 800 \\
\hline 40 & $32: 28$ & $32: 22$ & $32: 19$ & $32: 11$ & $32: 04$ & $40: 10$ & $39: 42$ & $39: 21$ & $39: 00$ & $38: 41$ \\
\hline 45 & $31: 42$ & $31: 37$ & $31: 33$ & $31: 29$ & $31: 24$ & $40: 05$ & $39: 38$ & $39: 16$ & $38: 56$ & $38: 38$ \\
\hline 50 & $31: 00$ & $30: 56$ & $30: 52$ & $30: 47$ & $30: 42$ & $40: 01$ & $39: 34$ & $39: 12$ & $38: 52$ & $38: 33$ \\
\hline 52 & $30: 44$ & $30: 38$ & $30: 34$ & $30: 30$ & $30: 26$ & $39: 58$ & $39: 31$ & $39: 09$ & $38: 50$ & $38: 31$ \\
\hline 54 & $30: 27$ & $30: 22$ & $30: 18$ & $30: 13$ & $30: 09$ & $: 56$ & $: 29$ & $: 06$ & $: 47$ & $: 29$ \\
\hline 56 & $30: 10$ & $30: 04$ & $30: 00$ & $29: 57$ & $29: 52$ & $: 53$ & $: 26$ & $: 04$ & $: 45$ & $: 27$ \\
\hline 58 & $29: 53$ & $29: 48$ & $29: 44$ & $29: 40$ & $29: 35$ & $: 50$ & $: 23$ & $39: 01$ & $: 42$ & :24 \\
\hline 60 & $29: 36$ & $29: 30$ & $29: 26$ & $29: 22$ & $29: 18$ & $: 47$ & :20 & $38: 58$ & $: 38$ & :20 \\
\hline 62 & $29: 18$ & $29: 12$ & $29: 08$ & $29: 05$ & $29: 01$ & $: 43$ & $: 15$ & $: 52$ & $: 33$ & $: 16$ \\
\hline 64 & $29: 00$ & $28: 55$ & $28: 51$ & $28: 49$ & $28: 44$ & $: 40$ & $: 10$ & $: 47$ & $: 29$ & $: 11$ \\
\hline 66 & $28: 41$ & $28: 36$ & $28: 33$ & $28: 30$ & $28: 27$ & $: 35$ & $: 05$ & :42 & $: 24$ & $: 07$ \\
\hline 68 & $28: 22$ & $28: 18$ & $28: 15$ & $28: 12$ & $28: 09$ & :29 & $39: 00$ & :38 & :19 & $38: 02$ \\
\hline 70 & $28: 03$ & $28: 00$ & $27: 56$ & $27: 54$ & $27: 52$ & $: 23$ & $38: 55$ & :32 & :13 & $37: 57$ \\
\hline 72 & $27: 45$ & $27: 42$ & $27: 40$ & $27: 39$ & $27: 37$ & :16 & $: 49$ & $: 27$ & $: 08$ & $: 51$ \\
\hline 74 & $27: 28^{*}$ & $27: 25$ & $27: 23$ & $27: 22$ & $27: 20$ & $: 10^{*}$ & $: 43$ & $: 21$ & $38: 02$ & $: 46$ \\
\hline 76 & $27: 10$ & $27: 07 *$ & $27: 06 *$ & $27: 05^{*}$ & $27: 04$ & :04 & $: 36^{*}$ & $: 15^{*}$ & $37: 56^{*}$ & $: 40$ \\
\hline 78 & $26: 58$ & $26: 55$ & $26: 53$ & $26: 51$ & $26: 48^{*}$ & :03 & $: 35$ & $: 12$ & $: 52$ & $: 34^{*}$ \\
\hline 80 & $26: 47$ & $26: 43$ & $26: 41$ & $26: 38$ & $26: 36$ & :02 & $: 34$ & $: 11$ & $: 51$ & :33 \\
\hline 82 & $26: 34$ & $26: 31$ & $26: 29$ & $26: 27$ & $26: 25$ & $39: 01$ & :32 & :09 & :50 & :32 \\
\hline 84 & $26: 22$ & $26: 19$ & $26: 17$ & $26: 15$ & $26: 13$ & $38: 59$ & $: 30$ & $: 07$ & $: 48$ & $: 30$ \\
\hline 86 & $26: 10$ & $26: 07$ & $26: 04$ & $26: 03$ & $26: 02$ & $: 57$ & $: 28$ & $: 05$ & $: 46$ & :28 \\
\hline 88 & $25: 58$ & $25: 55$ & $25: 52$ & $25: 51$ & $25: 51$ & $: 55$ & $: 26$ & $: 03$ & $: 43$ & $: 26$ \\
\hline 90 & $25: 45$ & $25: 43$ & $25: 41$ & $25: 40$ & $25: 40$ & $: 52$ & $: 24$ & $38: 01$ & $: 41$ & $: 24$ \\
\hline 95 & $25: 17$ & $25: 13$ & $25: 12$ & $25: 11$ & $25: 10$ & $: 45$ & :17 & $37: 54$ & :34 & $: 17$ \\
\hline 100 & $24: 48$ & $24: 45$ & $24: 43$ & $24: 42$ & $24: 41$ & $: 38$ & $: 10$ & $: 48$ & :28 & :10 \\
\hline 105 & $24: 18$ & $24: 16$ & $24: 14$ & $24: 13$ & $24: 12$ & $: 29$ & $38: 02$ & $: 40$ & $: 20$ & $37: 03$ \\
\hline 110 & $23: 46$ & $23: 43$ & $23: 41$ & $23: 40$ & $23: 39$ & $: 19$ & $37: 53$ & :31 & :10 & $36: 53$ \\
\hline 115 & $23: 14$ & $23: 11$ & 23:09 & $23: 08$ & $23: 07$ & $38: 09$ & $: 43$ & :21 & $37: 00$ & $: 42$ \\
\hline 120 & $22: 40$ & $22: 37$ & $22: 35$ & $22: 34$ & $22: 33$ & $37: 58$ & :31 & $: 08$ & $36: 48$ & :30 \\
\hline \multicolumn{11}{|c|}{$\mathrm{P}_{2}^{\prime} \mathrm{P}_{2}^{\prime}$} \\
\hline 40 & & & & & & $42: 05$ & $41: 33$ & $41: 12$ & $40: 54$ & $40: 38$ \\
\hline 50 & & & & & & 41:17 & $40: 45$ & $40: 24$ & $40: 06$ & $39: 50$ \\
\hline 60 & & & & & & $40: 28$ & $39: 56$ & $39: 35$ & $39: 17$ & $39: 01$ \\
\hline 70 & & & & & & $39: 40$ & $39: 08$ & $38: 46$ & $38: 28$ & $38: 12$ \\
\hline
\end{tabular}


TABLE 36

Differences in Travel Times, $\mathrm{P}^{\prime} \mathbf{P}^{\prime} \mathbf{P}^{\prime}-\mathrm{P}$, and Travel Times of $\mathbf{P}^{\prime} \mathbf{P}^{\prime} \mathrm{P}^{\prime}$ in Min.: Sec. for Various Depths of Focus in KM. and Distances $\Delta$ in Degrees (The first three values in each column concern the diffracted $\mathbf{P}^{\prime} \mathbf{P}^{\prime} \mathbf{P}^{\prime}$.)

\begin{tabular}{|c|c|c|c|c|c|c|c|c|}
\hline \multirow{2}{*}{$\Delta$} & \multicolumn{3}{|c|}{$P^{\prime} P^{\prime} P^{\prime}-P$ for depth of focus } & \multicolumn{5}{|c|}{$P^{\prime} P^{\prime} P^{\prime}-O$ for depth of focus } \\
\hline & 0 & 400 & 800 & 0 & 200 & 400 & 600 & 800 \\
\hline 45 . & $50: 00$ & $49: 50$ & $49: 41$ & $58: 21$ & $57: 54$ & $57: 32$ & $57: 11$ & $56: 55$ \\
\hline 60 . & $48: 22$ & $48: 13$ & 48:04 & $58: 33$ & $58: 06$ & $57: 44$ & $57: 23$ & $57: 06$ \\
\hline 65. & $48: 47$ & $48: 43$ & $48: 33$ & $: 35$ & $: 08$ & $: 46$ & $: 25$ & $: 08$ \\
\hline 70 . & $48: 28$ & $48: 27$ & $48: 19$ & $58: 48$ & $: 22$ & $58: 02$ & $: 40$ & $: 24$ \\
\hline 75 & $47: 15$ & $47: 12$ & $47: 07$ & $59: 03$ & $: 36$ & $: 16$ & $57: 55$ & $: 39$ \\
\hline 80 & $47: 01$ & $46: 58$ & $46: 55$ & :18 & $: 49$ & 29 & $58: 08$ & $57: 52$ \\
\hline 85. & $46: 46$ & $46: 44$ & $46: 41$ & :29 & $59: 01$ & :40 & $: 20$ & $58: 03$ \\
\hline 90. & $46: 32$ & $46: 30$ & $46: 28$ & :39 & :11 & :50 & 29 & :13 \\
\hline 95 & $46: 17$ & $46: 15$ & $46: 14$ & $: 47$ & :19 & $58: 58$ & $: 37$ & 21 \\
\hline 100. & $46: 02$ & $46: 01$ & $45: 59$ & $59: 54$ & :26 & $59: 05$ & :44 & $: 27$ \\
\hline 105 & $45: 47$ & $45: 45$ & $45: 44$ & $60: 00$ & :33 & :11 & :51 & :34 \\
\hline
\end{tabular}

TABLE 37

Differences in Travel Times, SKPP'-P, and Travel Times of SKPP' in Min.:Sec. for Variods Depths of Focus in KM. and Distances $\Delta$ in Degrees

(The diffracted SKPP' arrives at distances of $90^{\circ}$ and more. At these distances the travel times change more slowly than at shorter distances. PKSP' arrives later than

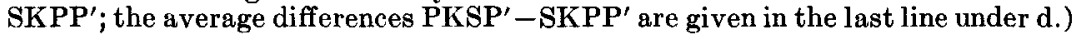

\begin{tabular}{|c|c|c|c|c|c|c|c|c|c|c|}
\hline \multirow{2}{*}{$\Delta$} & \multicolumn{5}{|c|}{$S K P P^{\prime}-P$ for depth of focus } & \multicolumn{5}{|c|}{ SKPP'-O for depth of focus } \\
\hline & 0 & 200 & 400 & 600 & 800 & 0 & 200 & 400 & 600 & 800 \\
\hline 55. & $33: 50$ & $33: 26$ & $33: 04$ & $32: 42$ & $32: 23$ & $43: 25$ & $42: 41$ & $42: 00$ & $41: 22$ & $40: 50$ \\
\hline 60. & $33: 07$ & $32: 42$ & $32: 21$ & $31: 58$ & $31: 40$ & $43: 18$ & $42: 32$ & $41: 52$ & $41: 14$ & $40: 42$ \\
\hline 65 & $32: 24$ & $32: 01$ & $31: 40$ & $31: 18$ & $30: 58$ & $43: 10$ & $42: 24$ & $41: 43$ & $41: 05$ & $40: 32$ \\
\hline 70. & $31: 42$ & $31: 19$ & $30: 58$ & $30: 38$ & $30: 17$ & $43: 01$ & $42: 14$ & $41: 34$ & $40: 54$ & $40: 22$ \\
\hline 75. & $31: 01$ & $30: 39$ & $30: 19$ & $29: 59$ & $29: 39$ & $42: 49$ & $42: 03$ & $41: 21$ & $40: 44$ & $40: 12$ \\
\hline 80. & $30: 20$ & $30: 00$ & $29: 39$ & $29: 19$ & $29: 01$ & $42: 36$ & $41: 51$ & $41: 09$ & $40: 32$ & $39: 58$ \\
\hline 85. & $29: 39$ & $29: 19$ & $28: 58$ & $28: 38$ & $28: 22$ & $42: 21$ & $41: 35$ & $40: 53$ & $40: 16$ & $39: 44$ \\
\hline
\end{tabular}

d

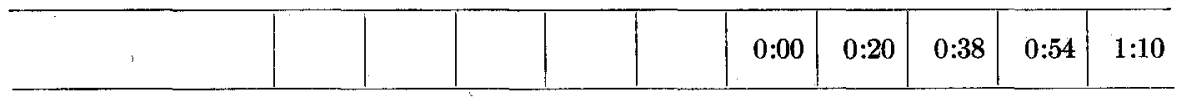


TABLE 38

Travel Times of PeP in Min.: Sec.

\begin{tabular}{|c|c|c|c|c|c|c|c|c|}
\hline \multirow{2}{*}{$\begin{array}{l}\text { Distance } \\
\text { in deg. }\end{array}$} & \multicolumn{8}{|c|}{ Depth of focus in km. } \\
\hline & 100 & 200 & 300 & 400 & 500 & 600 & 700 & 800 \\
\hline $0 \ldots \ldots$ & $8: 23$ & $8: 11$ & $8: 00$ & $7: 48$ & $7: 37$ & $7: 27$ & $7: 17$ & 7:08 \\
\hline $10 \ldots \ldots \ldots$ & $8: 24$ & $8: 12$ & 8:01 & $7: 49$ & $7: 38$ & $7: 28$ & $7: 18$ & $7: 09$ \\
\hline $20 \ldots \ldots \ldots$ & $8: 36$ & $8: 24$ & $8: 13$ & 8:02 & $7: 52$ & $7: 42$ & $7: 32$ & $7: 24$ \\
\hline $30 \ldots$ & $8: 59$ & $8: 47$ & $8: 36$ & $8: 25$ & $8: 15$ & $8: 05$ & $7: 55$ & $7: 47$ \\
\hline $40 .$. & $9: 29$ & $9: 17$ & $9: 06$ & $8: 54$ & $8: 44$ & $8: 34$ & $8: 25$ & $8: 17$ \\
\hline 45. & $9: 47$ & $9: 35$ & $9: 24$ & $9: 13$ & $9: 03$ & $8: 53$ & $8: 44$ & $8: 36$ \\
\hline 50. & $10: 06$ & $9: 54$ & $9: 43$ & $9: 33$ & $9: 23$ & $9: 13$ & $9: 04$ & $8: 56$ \\
\hline $55 \ldots \ldots \ldots$ & $10: 26$ & $10: 14$ & $10: 03$ & $9: 53$ & $9: 43$ & $9: 33$ & $9: 24$ & $9: 16$ \\
\hline 60. & $10: 46$ & $10: 34$ & $10: 23$ & $10: 13$ & $10: 03$ & $9: 53$ & $9: 44$ & 9:36 \\
\hline 65. & $11: 07$ & $10: 55$ & $10: 44$ & $10: 34$ & $10: 24$ & $10: 14$ & $10: 05$ & $9: 57$ \\
\hline 70. & $11: 28$ & $11: 16$ & $11: 05$ & $10: 55$ & $10: 45$ & $10: 36$ & $10: 27$ & $10: 19$ \\
\hline $75 \ldots$ & $11: 49$ & $11: 37$ & $11: 26$ & $11: 16$ & $11: 06$ & $10: 57$ & $10: 48$ & $10: 40$ \\
\hline 80 . & $12: 10$ & $11: 58$ & $11: 47$ & $11: 37$ & $11: 27$ & $11: 18$ & $11: 09$ & $11: 01$ \\
\hline 85. & $12: 32$ & $12: 20$ & $12: 09$ & $11: 59$ & $11: 49$ & $11: 40$ & $11: 31$ & $11: 23$ \\
\hline $90 \ldots$ & $12: 54$ & $12: 42$ & $12: 31$ & $12: 21$ & $12: 11$ & $12: 02$ & $11: 53$ & $11: 45$ \\
\hline
\end{tabular}

TABLE 39

Differences in Travel Times, pPcP-PcP, sPcP-PcP, and PeP-P, in Min.: Sec.

\begin{tabular}{|c|c|c|c|c|c|c|c|c|}
\hline \multirow{2}{*}{$\begin{array}{l}\text { Distance } \\
\text { in deg. }\end{array}$} & \multicolumn{8}{|c|}{ Depth of focus in $\mathrm{km}$. } \\
\hline & 100 & 200 & 300 & 400 & 500 & 600 & 700 & 800 \\
\hline \multicolumn{9}{|c|}{$\mathrm{pPcP}-\mathrm{PcP}$} \\
\hline 0 . & $0: 30$ & $0: 54$ & $1: 17$ & $1: 40$ & $2: 02$ & $2: 23$ & $2: 42$ & $3: 00$ \\
\hline 30. & $0: 28$ & $0: 52$ & $1: 14$ & $1: 36$ & $1: 57$ & $2: 16$ & $2: 36$ & $2: 52$ \\
\hline 60. & $0: 27$ & $0: 51$ & $1: 13$ & $1: 33$ & $1: 53$ & $2: 12$ & $2: 31$ & $2: 47$ \\
\hline $90 \ldots$ & $0: 26$ & $0: 50$ & $1: 12$ & $1: 32$ & $1: 52$ & $2: 10$ & $2: 28$ & $2: 44$ \\
\hline \multicolumn{9}{|c|}{$\mathrm{sPcP}-\mathrm{PcP}$} \\
\hline 0. & $0: 39$ & $1: 14$ & $1: 47$ & $2: 18$ & $2: 48$ & $3: 16$ & $3: 44$ & $4: 11$ \\
\hline 90. & $0: 37$ & $1: 12$ & $1: 44$ & $2: 15$ & $2: 42$ & $3: 09$ & $3: 35$ & $4: 00$ \\
\hline \multicolumn{9}{|c|}{$\mathrm{PcP}-\mathrm{P}$} \\
\hline 50. & $1: 19$ & $1: 17$ & $1: 15$ & $1: 13$ & $1: 11$ & $1: 08$ & $1: 06$ & $1: 05$ \\
\hline 60. & $0: 46$ & $0: 45$ & $0: 43$ & $0: 42$ & $0: 40$ & $0: 38$ & $0: 36$ & $0: 34$ \\
\hline 70. & $0: 21$ & $0: 21$ & $0: 21$ & $0: 20$ & $0: 19$ & $0: 18$ & $0: 16$ & $0: 14$ \\
\hline $80 \ldots$ & $0: 07$ & $0: 07$ & $0: 07$ & $0: 07$ & $0: 06$ & $0: 05$ & $0: 04$ & $0: 04$ \\
\hline
\end{tabular}


of the type of $\mathrm{pS}, \mathrm{PS}, \mathrm{pSS}$, etc., where the $\mathrm{S}$ wave leaves the point of reflection more steeply than the longitudinal wave arrives, there will be a certain depth for which the ray reaching the critical epicentral distance just grazes the core; for hypocenters deeper than this, the phase in question cannot exist. This is the case for pS and PS when the hypocenter is deeper than about $900 \mathrm{~km}$.

Tables 6 to 44 can be used for other constructions; thus, curves can be plotted showing the variation of travel times with depth of focus, at a given distance. A third possible construction is that showing the variation of depth and distance for constant travel time; in polar coördinates the curves are wave fronts. It is

TABLE 40

Travel Times of ScS in Mrn.: Sec.

\begin{tabular}{|c|c|c|c|c|c|c|c|c|}
\hline \multirow{2}{*}{$\begin{array}{l}\text { Distance } \\
\text { in deg. }\end{array}$} & \multicolumn{8}{|c|}{ Depth of focus in $\mathrm{km}$. } \\
\hline & 100 & 200 & 300 & 400 & 500 & 600 & 700 & 800 \\
\hline & $15: 14$ & $14: 51$ & $14: 30$ & $14: 09$ & $13: 49$ & $13: 32$ & $13: 14$ & $12: 57$ \\
\hline 5. & $: 16$ & $14: 53$ & $: 32$ & 12 & :52 & $: 35$ & $: 17$ & $13: 01$ \\
\hline 10. & $: 23$ & $15: 00$ & $: 39$ & :19 & $13: 59$ & :42 & $: 25$ & $: 09$ \\
\hline 15. & :33 & :11 & $14: 49$ & $: 30$ & 14:10 & $13: 53$ & $: 36$ & $: 20$ \\
\hline 20 . & $: 46$ & :25 & $15: 03$ & $: 44$ & $: 25$ & $14: 08$ & $13: 51$ & :35 \\
\hline 22. & $15: 53$ & :31 & :09 & :50 & :31 & $: 15$ & $13: 59$ & :44 \\
\hline 24. & $16: 00$ & :38 & :17 & $14: 58$ & :39 & $: 22$ & 14:06 & $13: 52$ \\
\hline 26 . & $: 08$ & $: 46$ & :25 & $15: 05$ & $: 47$ & :30 & :14 & $14: 00$ \\
\hline 28. & 16 & $15: 54$ & :34 & :14 & $14: 57$ & $: 39$ & :23 & $: 09$ \\
\hline 30. & $: 25$ & $16: 03$ & $: 43$ & $: 24$ & $15: 06$ & $: 49$ & :33 & :18 \\
\hline 32 . & $: 36$ & :14 & $15: 54$ & $: 34$ & :16 & $14: 59$ & $: 43$ & 28 \\
\hline 34. & $: 47$ & $: 25$ & $16: 05$ & $: 45$ & :26 & $15: 09$ & $14: 54$ & $: 39$ \\
\hline 36. & $16: 58$ & $: 37$ & $: 16$ & $15: 56$ & $: 37$ & $: 20$ & $15: 04$ & $14: 50$ \\
\hline 38. & $17: 09$ & $16: 48$ & $: 28$ & $16: 08$ & $15: 49$ & $: 32$ & 16 & $15: 02$ \\
\hline 40. & $: 20$ & $17: 00$ & $: 40$ & $: 20$ & $16: 02$ & $: 45$ & $: 29$ & :15 \\
\hline 42. & $: 32$ & :12 & $16: 52$ & :32 & :14 & $15: 57$ & $: 41$ & $: 27$ \\
\hline 44. & $: 44$ & :24 & $17: 04$ & $: 45$ & $: 27$ & $16: 10$ & $: 53$ & :39 \\
\hline 46. & $17: 56$ & $: 36$ & $: 16$ & $16: 57$ & $: 39$ & $: 22$ & $16: 05$ & $15: 51$ \\
\hline 48. & $18: 08$ & $: 48$ & $: 27$ & $17: 09$ & $16: 51$ & $: 34$ & :18 & $16: 04$ \\
\hline 50. & $18: 20$ & $18: 00$ & $17: 39$ & $17: 21$ & $17: 04$ & $16: 47$ & $16: 31$ & $16: 16$ \\
\hline 55. & 18:51 & $18: 31$ & $18: 11$ & $17: 53$ & $17: 35$ & $17: 17$ & $17: 02$ & $16: 48$ \\
\hline 60. & $19: 26$ & $19: 05$ & $18: 44$ & $18: 26$ & 18:08 & $17: 50$ & $17: 35$ & $17: 20$ \\
\hline 65. & $20: 03$ & $19: 43$ & $19: 23$ & 19:05 & $19: 47$ & $18: 29$ & $18: 13$ & $17: 58$ \\
\hline 70 . & $20: 41$ & $20: 21$ & $20: 01$ & $19: 43$ & $19: 26$ & $19: 08$ & $18: 53$ & $18: 39$ \\
\hline 75. & $21: 22$ & $21: 02$ & $20: 42$ & $20: 23$ & $20: 05$ & $19: 48$ & $19: 33$ & $19: 19$ \\
\hline 80 . & $22: 03$ & $21: 43$ & $21: 23$ & $21: 04$ & $20: 45$ & $20: 28$ & $20: 12$ & $19: 58$ \\
\hline
\end{tabular}


TABLE 41

Differences in Travel Times, pSeS-SeS, sSeS-SeS, and ScS-S, in Min.:Sec.

\begin{tabular}{|c|c|c|c|c|c|c|c|c|}
\hline \multirow{2}{*}{$\begin{array}{l}\text { Distance } \\
\text { in deg. }\end{array}$} & \multicolumn{8}{|c|}{ Depth of focus in $\mathrm{km}$. } \\
\hline & 100 & 200 & 300 & 400 & 500 & 600 & 700 & 800 \\
\hline \multicolumn{9}{|c|}{$\mathrm{pScS}-\mathrm{ScS}$} \\
\hline 0 . & $0: 39$ & $1: 14$ & $1: 47$ & $2: 18$ & $2: 48$ & $3: 16$ & $3: 44$ & $4: 11$ \\
\hline 100. & $0: 34$ & $1: 03$ & $1: 32$ & $1: 59$ & $2: 23$ & $2: 44$ & $3: 01$ & $3: 16$ \\
\hline \multicolumn{9}{|c|}{ sScS-ScS } \\
\hline 0 . & $0: 48$ & $1: 35$ & $2: 16$ & $2: 56$ & $3: 36$ & $4: 12$ & $4: 48$ & $5: 22$ \\
\hline 30 & $0: 48$ & $1: 31$ & $2: 11$ & $2: 50$ & $3: 28$ & 4:01 & $4: 33$ & $5: 04$ \\
\hline 60 & $0: 47$ & $1: 29$ & $2: 08$ & $2: 48$ & $3: 24$ & $3: 58$ & $4: 29$ & $5: 00$ \\
\hline 90 . & $0: 47$ & $1: 28$ & $2: 07$ & $2: 47$ & $3: 22$ & $3: 56$ & $4: 26$ & $4: 56$ \\
\hline \multicolumn{9}{|c|}{$\mathrm{ScS}-\mathrm{S}$} \\
\hline 50 . & $2: 33$ & $2: 32$ & $2: 27$ & $2: 24$ & $2: 21$ & $2: 19$ & $2: 15$ & $2: 13$ \\
\hline 60. & $1: 24$ & $1: 22$ & $1: 20$ & $1: 18$ & $1: 15$ & $1: 11$ & $1: 07$ & $1: 04$ \\
\hline 70 . & $0: 41$ & $0: 41$ & $0: 40$ & $0: 39$ & $0: 37$ & $0: 34$ & $0: 32$ & $0: 30$ \\
\hline $80 \ldots$ & $0: 11$ & $0: 11$ & 0:09 & $0: 07$ & $0: 05$ & 0:04 & $0: 03$ & $0: 02$ \\
\hline
\end{tabular}

TABLE 42

Travel Times of ScP in Min.: Sec., and Distance at Which the Curve Ends

\begin{tabular}{|c|c|c|c|c|c|c|c|c|}
\hline \multirow{2}{*}{$\begin{array}{l}\text { Distance } \\
\text { in deg. }\end{array}$} & \multicolumn{8}{|c|}{ Depth of focus in $\mathrm{km}$. } \\
\hline & 100 & 200 & 300 & 400 & 500 & 600 & 700 & 800 \\
\hline $0 \ldots \ldots \ldots \ldots$ & $11: 44$ & $11: 21$ & 11:00 & 10.40 & $10: 20$ & $10: 02$ & $9: 44$ & $9: 27$ \\
\hline $10 \ldots \ldots \ldots \ldots$ & $11: 49$ & $11: 25$ & $11: 05$ & $10: 44$ & $10: 25$ & $10: 07$ & $9: 50$ & $9: 32$ \\
\hline 20. & $12: 01$ & $11: 38$ & $11: 17$ & $10: 59$ & $10: 40$ & $10: 23$ & $10: 06$ & $9: 49$ \\
\hline $30 \ldots \ldots$ & $12: 29$ & $12: 07$ & $11: 45$ & $11: 26$ & $11: 08$ & $10: 51$ & $10: 35$ & $10: 19$ \\
\hline $40 \ldots \ldots \ldots \ldots$ & $13: 06$ & $12: 44$ & $12: 22$ & $12: 03$ & $11: 45$ & $11: 28$ & $11: 13$ & $10: 58$ \\
\hline $50 \ldots \ldots \ldots \ldots$ & $13: 47$ & $13: 24$ & $13: 03$ & $12: 44$ & $12: 26$ & $12: 10$ & $11: 55$ & $11: 41$ \\
\hline $60 \ldots$ & $14: 29$ & $14: 06$ & $13: 45$ & $13: 25$ & $13: 07$ & $12: 51$ & $12: 38$ & $12: 26$ \\
\hline \multicolumn{9}{|c|}{ End } \\
\hline & $66^{\circ}$ & $6512^{\circ}$ & $651 / 4^{\circ}$ & $65^{\circ}$ & $641 / 2{ }^{\circ}$ & $64^{\circ}$ & $631 / 2^{\circ}$ & $63^{\circ}$ \\
\hline
\end{tabular}


more convenient to use rectangular coördinates; if the abscissa is $\Delta$ in degrees, the ordinate should be the natural logarithm of the radius, multiplied by $\frac{180}{\pi}$. This is a conformal representation, so that the rays can be drawn orthogonal to the wave fronts.

A complete construction of the travel times would require a three-dimensional model.

TABLE 43

Travel Times of PeS in Min.: Sec.

(PcS ends at about the same distance as ScP.)

\begin{tabular}{c|c|c|c|c|c|c|c|c}
\hline \hline \multirow{2}{*}{$\begin{array}{c}\text { Distance } \\
\text { in deg. }\end{array}$} & \multicolumn{7}{|c|}{ Depth of focus in km. } \\
\cline { 2 - 9 } & 100 & 200 & 300 & 400 & 500 & 600 & 700 & 800 \\
\hline $0 \ldots \ldots \ldots \ldots \ldots$ & $11: 53$ & $11: 41$ & $11: 30$ & $11: 18$ & $11: 07$ & $10: 57$ & $10: 47$ & $10: 38$ \\
$10 \ldots \ldots \ldots \ldots \ldots$ & $11: 58$ & $11: 45$ & $11: 34$ & $11: 23$ & $11: 12$ & $11: 02$ & $10: 52$ & $10: 43$ \\
$20 \ldots \ldots \ldots \ldots \ldots$ & $12: 12$ & $11: 58$ & $11: 47$ & $11: 36$ & $11: 25$ & $11: 15$ & $11: 05$ & $10: 56$ \\
$30 \ldots \ldots \ldots \ldots \ldots$ & $12: 39$ & $12: 26$ & $12: 15$ & $12: 04$ & $11: 54$ & $11: 44$ & $11: 35$ & $11: 26$ \\
& & & & & & & & \\
$40 \ldots \ldots \ldots \ldots \ldots$ & $13: 16$ & $13: 03$ & $12: 52$ & $12: 42$ & $12: 32$ & $12: 22$ & $12: 14$ & $12: 04$ \\
$50 \ldots \ldots \ldots \ldots$ & $13: 57$ & $13: 45$ & $13: 34$ & $13: 24$ & $13: 13$ & $13: 04$ & $12: 55$ & $12: 46$ \\
$60 \ldots \ldots \ldots \ldots \ldots$ & $14: 39$ & $14: 27$ & $14: 16$ & $14: 06$ & $13: 56$ & $13: 47$ & $13: 38$ & $13: 29$ \\
\hline
\end{tabular}

TABLE 44

Differences in Travel Times, sScP-ScP and pPcS-PeS, in Min.: Sec.

(The travel times of $\mathrm{sPcS}$ are the same as those of $\mathrm{sScP}$, and the travel times of $\mathrm{pScP}$ are the same as those of $\mathrm{pPcS}$.)

\begin{tabular}{|c|c|c|c|c|c|c|c|c|}
\hline \multirow{2}{*}{$\begin{array}{l}\text { Distance } \\
\text { in deg. }\end{array}$} & \multicolumn{8}{|c|}{ Depth of focus in $\mathrm{km}$. } \\
\hline & 100 & 200 & 300 & 400 & 500 & 600 & 700 & 800 \\
\hline \multicolumn{9}{|c|}{$\mathrm{sScP}-\mathrm{ScP}$} \\
\hline $0 \ldots \ldots \ldots \ldots$ & $0: 47$ & $1: 35$ & $2: 16$ & $2: 56$ & $3: 36$ & $4: 12$ & $4: 48$ & $5: 22$ \\
\hline $60 \ldots$ & $0: 46$ & $1: 32$ & $2: 13$ & $2: 52$ & $3: 30$ & $4: 02$ & $4: 28$ & $4: 52$ \\
\hline \multicolumn{9}{|c|}{$\mathrm{pPcS}-\mathrm{PcS}$} \\
\hline $0 \ldots \ldots$ & $0: 30$ & $0: 54$ & $1: 17$ & $1: 40$ & $2: 02$ & $2: 23$ & $2: 42$ & $3: 00$ \\
\hline $60 \ldots \ldots$ & $0: 27$ & $0: 51$ & $1: 12$ & $1: 32$ & $1: 51$ & $2: 10$ & $2: 28$ & $2: 45$ \\
\hline
\end{tabular}




\section{Location of EpICENTERS}

In order to test the travel times given above, the times of certain deep-focus shocks have been investigated in detail. The data have been taken from the International Seismological Summary, from station bulletins, and from the original seismograms written at Pasadena and Huancayo. Use has also been made of shocks for which the data have been published by other investigators.

Where it was necessary to determine or to revise the epicenter, a procedure was adopted which had previously been used with success in the study of normal earthquakes. ${ }^{3}$

The first step is to select pairs of reliable stations, differing as widely as possible in azimuth, for which the times of $\mathrm{P}$ are separated by small intervals (preferably not more than ten seconds).

Having an approximate epicenter, and data for a given pair of stations, we examine the relative positions on a globe. The difference in time between the two stations corresponds to a certain difference in distance, $\delta \Delta$. Unless the time difference is large, this will not depend much on the focal depth.

We now replace the coördinates of the nearer station by those of a point found by receding from the station a distance $\delta \Delta$ directly away from the epicenter, or we advance from the more distant station a distance $\delta \Delta$ directly toward the epicenter. In this way we find the coördinates of two points, the first being one of the two stations, the second replacing the other station; these points are at equal distances from the corrected epicenter, which consequently must lie on the great circle which perpendicularly bisects the great-circle arc joining the two points. Each pair of stations thus determines a great circle passing through the epicenter.

A numerical second approximation to the coördinates of the epicenter can now be found by the method of least squares. To set up the appropriate equations, a central projection is used, as suggested by Galitzin. ${ }^{4}$ The origin of projection is the center of the earth, and the plane of projection is parallel to the equatorial plane. The equations of this projection are

$$
x=\cot \phi \cos \lambda, y=\cot \phi \sin \lambda
$$

where $\phi$ and $\lambda$ are latitude and longitude, as usual. This projection has the advantage that all great circles are transformed into straight lines (the equator is at infinity).

${ }^{3}$ B. Gutenberg, "Die mitteleuropäischen Beben ... I," Bearbeitung der instrumentellen A ufzeichnungen, Veröff. Zentralbüro der Internat. Seismol. Assoc. (Strassburg, 1915).

${ }^{4}$ B. Galitzin, "Zur Frage der Bestimmung der Herdtiefe eines Bebens und der Fortpflanzungsgeschwindigkeit der seismischen Wellen ....," Nachr. der seismol. Kommission, 5:359-430 (1912). 
The equation of the straight line representing the great circle equidistant from two points $\left(\phi_{1}, \lambda_{1}\right.$ and $\left.\phi_{2}, \lambda_{2}\right)$ is

$\left(\cos \phi_{1} \cos \lambda_{1}-\cos \phi_{2} \cos \lambda_{2}\right) x+\left(\cos \phi_{1} \sin \lambda_{1}-\cos \phi_{2} \sin \lambda_{2}\right) y+\left(\sin \phi_{1}-\sin \phi_{2}\right)=0$

Each pair of stations gives an equation of this type; since these are linear in $x$ and $y$, the entire system can be solved by the method of least squares. If the solution is found in the form

$$
x=\mathrm{A} \pm a, y=B \pm b
$$

then the desired coördinates $\Phi, \Lambda$, with their mean errors, are given by

$$
\begin{aligned}
& \cot \Phi=\sqrt{A^{2}+B^{2}} \pm \sqrt{\frac{a^{2} A^{2}+b^{2} B^{2}}{A^{2}+B_{2}}} \\
& \tan \Lambda=\frac{B}{A} \pm \frac{1}{A^{2}} \sqrt{b^{2} A^{2}+a^{2} B^{2}}
\end{aligned}
$$

The labor of applying the method of least squares can be reduced by using as a preliminary approximation coördinates found by plotting the straight lines given by equations of type (2).

\section{Determination of Origin Time}

In the previous paper ${ }^{5}$ it has been pointed out that $\mathbf{P}-\mathrm{O}$ as a function of $\mathbf{S}-\mathbf{P}$ does not depend very much on focal depth. By using tables 6 and 15 it is now possible to calculate the $\mathrm{P}-\mathrm{O}$ corresponding to a given $\mathrm{S}-\mathrm{P}$ for various depths. The results are given in table 45 . From the table it will be seen that the effect of depth is appreciable only for the greatest depths used, and even then only for distances of more than $60^{\circ}$. Consequently, the use of $\mathrm{S}-\mathrm{P}$, as tabulated for normal shocks, to determine $\mathrm{P}-\mathrm{O}$ for deep-focus shocks, will yield a reasonably good first approximation to the origin time. However, this method is subject to the well-known uncertainty in identifying the correct $\mathrm{S}$, even if the depth is known and correct $\mathrm{P}-\mathrm{O}$ is taken from table 45.

Sohon ${ }^{6}$ has suggested a refinement of this procedure, in which the time of $\mathbf{S}$ is replaced by the arithmetic mean of the times of $\mathrm{S}$ and $\mathrm{sS}$, and the time of $\mathrm{P}$ is replaced by the mean of $\mathrm{P}$ and $\mathrm{pP}$. In using this to improve the $\mathrm{S}-\mathrm{P}$ method, there is still uncertainty concerning the identification of $\mathbf{S}, \mathrm{sS}$, and sometimes of $\mathrm{pP}$.

When the epicenter has been determined otherwise, the Sohon method gives a very precise determination of the origin time, since approximately

$$
O=(p P+P) / 2-t_{0}
$$

where $t_{0}$ is the travel time of $\mathrm{P}$ waves $(\mathrm{P}-\mathrm{O})$ for zero depth and the same epicentral distance. Calculation shows that, down to hypocentral depths of 800

${ }^{5}$ B. Gutenberg and C. F. Richter, "Contribution to the Study of Deep-Focus Earthquakes," Gerlands Beitr. z. Geophys., 41:160-169 (1934).

6 F. W. Sohon, "A First Approximation for Deep-Focus Seismograms," Bull. Seism. Soc. Am., 25:311 (1935). 
TABLE 45

Distance Corresponding to a Given Time Interval S-P, in Min:: Sec., for Shallow Earthquakes and Time Interval P-O in Min.:Sec. for Earthquakes With Various Depths

\begin{tabular}{|c|c|c|c|c|c|c|}
\hline \multirow{2}{*}{$\mathrm{S}-\mathrm{P}$} & \multirow{2}{*}{$\begin{array}{l}\text { Distance } \\
\text { in deg. }\end{array}$} & \multicolumn{5}{|c|}{$\mathrm{P}-\mathrm{O}$ for depth of focus in $\mathrm{km}$. } \\
\hline & & 25 & 200 & 400 & 600 & 800 \\
\hline $1: 00$ & 4.9 & $1: 18$ & $1: 18$ & $1: 18$ & $1: 18$ & $1: 17$ \\
\hline$: 10$ & 5.8 & $1: 30$ & $1: 30$ & $1: 30$ & $1: 30$ & $1: 29$ \\
\hline$: 20$ & 6.7 & $1: 43$ & $1: 42$ & $1: 43$ & $1: 43$ & $1: 42$ \\
\hline$: 30$ & 7.6 & $1: 55$ & $1: 54$ & $1: 56$ & $1: 56$ & $1: 54$ \\
\hline$: 40$ & 8.6 & $2: 07$ & 2:06 & $2: 09$ & $2: 08$ & $2: 07$ \\
\hline$: 50$ & 9.5 & $2: 20$ & 2:19 & $2: 22$ & $2: 21$ & $2: 20$ \\
\hline 2:00 & 10.4 & $2: 33$ & $2: 32$ & $2: 34$ & $2: 34$ & $2: 30$ \\
\hline :10 & 11.3 & $2: 46$ & $2: 46$ & $2: 47$ & $2: 47$ & $2: 43$ \\
\hline$: 20$ & 12.2 & $2: 58$ & 3:00 & $3: 00$ & $3: 00$ & $2: 56$ \\
\hline$: 30$ & 13.1 & $3: 10$ & $3: 14$ & $3: 13$ & $3: 13$ & $3: 09$ \\
\hline$: 40$ & 13.9 & $3: 22$ & $3: 27$ & $3: 25$ & $3: 25$ & $3: 21$ \\
\hline$: 50$ & 14.8 & $3: 34$ & $3: 39$ & $3: 36$ & $3: 38$ & $3: 34$ \\
\hline $3: 00$ & 15.8 & $3: 46$ & $3: 50$ & $3: 47$ & $3: 49$ & $3: 47$ \\
\hline :10 & 16.8 & $3: 58$ & $4: 00$ & $3: 59$ & $4: 00$ & $3: 59$ \\
\hline$: 20$ & 17.8 & $4: 10$ & $4: 11$ & $4: 10$ & $4: 10$ & $4: 11$ \\
\hline :30. & 18.8 & $4: 22$ & $4: 22$ & $4: 22$ & $4: 21$ & $4: 24$ \\
\hline$: 40$ & 19.9 & $4: 35$ & $4: 34$ & $4: 34$ & $4: 33$ & $4: 36$ \\
\hline$: 50$ & 21.0 & $4: 48$ & $4: 47$ & $4: 46$ & $4: 45$ & $4: 49$ \\
\hline 4:00 & 22.2 & $5: 00$ & - $5: 00$ & $4: 59$ & $4: 58$ & $5: 03$ \\
\hline$: 10$ & 23.5 & $5: 12$ & $5: 12$ & $5: 11$ & $5: 11$ & $5: 16$ \\
\hline$: 20$ & 24.7 & $5: 24$ & $5: 25$ & $5: 23$ & $5: 24$ & $5: 30$ \\
\hline$: 30$ & 25.9 & $5: 35$ & $5: 38$ & $5: 36$ & $5: 38$ & $5: 45$ \\
\hline$: 40$ & 27.1 & $5: 46$ & $5: 51$ & $5: 50$ & $5: 53$ & $5: 59$ \\
\hline$: 50$ & 28.4 & $5: 58$ & $6: 04$ & $6: 02$ & $6: 09$ & $6: 13$ \\
\hline 5:00 & 29.9 & $6: 11$ & $6: 16$ & $6: 15$ & $6: 24$ & $6: 24$ \\
\hline$: 10$ & 31.4 & $6: 25$ & $6: 28$ & $6: 28$ & $6: 37$ & $6: 34$ \\
\hline$: 20$ & 33.0 & $6: 38$ & $6: 41$ & $6: 42$ & $6: 49$ & $6: 44$ \\
\hline$: 30$ & 34.6 & $6: 52$ & $6: 54$ & $6: 55$ & $7: 00$ & $6: 54$ \\
\hline$: 40$ & 36.2 & $7: 05$ & $7: 08$ & $7: 07$ & $7: 11$ & $7: 05$ \\
\hline :50. & 37.7 & $7: 19$ & $7: 21$ & $7: 20$ & $7: 23$ & $7: 16$ \\
\hline $6: 00$ & 39.3 & $7: 34$ & $7: 34$ & $7: 33$ & $7: 35$ & $7: 28$ \\
\hline :10 & 41.0 & $7: 49$ & $7: 47$ & $7: 46$ & $7: 49$ & $7: 41$ \\
\hline :20 & 42.8 & $8: 03$ & $8: 00$ & $7: 59$ & 8:01 & $7: 54$ \\
\hline :30 & 44.4 & $8: 15$ & $8: 13$ & $8: 12$ & $8: 12$ & $8: 08$ \\
\hline$: 40$ & 45.9 & $8: 27$ & $8: 26$ & $8: 24$ & $8: 23$ & $8: 21$ \\
\hline$: 50$ & 47.4 & $8: 40$ & 8:38 & $8: 37$ & $8: 35$ & $8: 34$ \\
\hline
\end{tabular}


TABLE 45-(Concluded)

\begin{tabular}{|c|c|c|c|c|c|c|}
\hline \multirow{2}{*}{$\mathbf{S - P}$} & \multirow{2}{*}{$\begin{array}{l}\text { Distance } \\
\text { in deg. }\end{array}$} & \multicolumn{5}{|c|}{$P-O$ for depth of focus in $\mathrm{km}$. } \\
\hline & & 25 & 200 & 400 & 600 & 800 \\
\hline 7:00 & 49.0 & $8: 52$ & $8: 51$ & $8: 50$ & $8: 46$ & $8: 47$ \\
\hline i0 & 50.5 & 9:03 & 9:02 & 9:01 & $8: 58$ & 9:00 \\
\hline :20 & 52.0 & $9: 13$ & $9: 14$ & $9: 12$ & $9: 11$ & 9:13 \\
\hline :30 & 53.4 & $9: 22$ & $9: 26$ & $9: 24$ & $9: 24$ & $9: 26$ \\
\hline :40 & 54.8 & $9: 32$ & $9: 36$ & $9: 35$ & $9: 36$ & $9: 38$ \\
\hline :50 & 56.3 & $9: 43$ & $9: 46$ & $9: 46$ & $9: 47$ & $9: 50$ \\
\hline $8: 00$ & 58.0 & $9: 55$ & $9: 57$ & $9: 58$ & 9:58 & $10: 01$ \\
\hline :10 & 59.9 & 10:09 & 10:09 & $10: 10$ & $10: 10$ & $10: 11$ \\
\hline :20 & 61.9 & $10: 23$ & $10: 22$ & $10: 23$ & $10: 21$ & $10: 20$ \\
\hline :30 & 63.9 & $10: 36$ & $10: 35$ & $10: 37$ & $10: 32$ & $10: 28$ \\
\hline :40 & 65.8 & $10: 48$ & $10: 48$ & $10: 49$ & $10: 42$ & $10: 36$ \\
\hline$: 50$ & 67.8 & 11:00 & 11:00 & $10: 59$ & $10: 52$ & $10: 45$ \\
\hline $9: 00$ & 69.7 & $11: 12$ & $11: 12$ & $11: 08$ & $11: 03$ & $10: 55$ \\
\hline 10 & 71.5 & $11: 24$ & $11: 22$ & $11: 17$ & $11: 13$ & $11: 05$ \\
\hline 20 & 73.4 & $11: 35$ & $11: 32$ & $11: 26$ & $11: 22$ & $11: 15$ \\
\hline 30 & 75.3 & $11: 46$ & 11:41 & $11: 35$ & $11: 31$ & $11: 24$ \\
\hline :40 & 77.2 & $11: 56$ & $11: 51$ & $11: 44$ & $11: 40$ & $11: 34$ \\
\hline :50 & 79.1 & 12:06 & 12:01 & 11:53 & $11: 49$ & $11: 44$ \\
\hline $10: 00$ & 80.8 & $12: 15$ & $12: 10$ & $12: 02$ & $11: 59$ & $11: 54$ \\
\hline :10 & 82.4 & $12: 24$ & $12: 20$ & $12: 12$ & $12: 10$ & $12: 05$ \\
\hline :20 & 84.0 & $12: 32$ & $12: 29$ & $12: 23$ & $12: 21$ & $12: 16$ \\
\hline :30 & 85.7 & $12: 41$ & $12: 38$ & $12: 34$ & $12: 32$ & $12: 27$ \\
\hline :40 & 87.5 & $12: 50$ & $12: 48$ & $12: 45$ & $12: 43$ & $12: 38$ \\
\hline :50 & 89.6 & 13:00 & $12: 58$ & $12: 55$ & $12: 54$ & $12: 48$ \\
\hline $11: 00$ & 91.9 & $13: 11$ & $13: 09$ & $13: 05$ & $13: 05$ & $12: 58$ \\
\hline :10 & 94.4 & $13: 22$ & $13: 19$ & $13: 16$ & $13: 16$ & $13: 08$ \\
\hline$: 20$ & 97.0 & $13: 33$ & $13: 30$ & $13: 27$ & $13: 27$ & $13: 18$ \\
\hline :30 & 99.5 & $13: 45$ & $13: 41$ & 13:39 & $13: 38$ & $13: 29$ \\
\hline
\end{tabular}

$\mathrm{km}$., this procedure will give origin times which agree within a very few seconds with the data of tables 6,7 , and 8 .

In order to avoid the necessity of constantly reducing from normal depth (assumed as $25 \mathrm{~km}$.) to zero depth, we have written the corresponding equation in the form

$$
O=(p P+P) / 2-t_{p}-c
$$

where $t_{p}$ is the travel time of $\mathrm{P}$ for a normal shock to the given epicentral distance, and where $c$ has the values given in table 46 . These tabulated values include the correction from zero to normal depth, as well as the small errors of Sohon's method. 
TABLE 46

Corrections $c$ (in Seconds) to be Used in Calcdlating the Origin Time O From $O=(p P+P / 2)-t_{p}-c$

( $t_{p}$ is the travel time of longitudinal waves in a shock with normal depth, supposed to be $25 \mathrm{~km}$.)

\begin{tabular}{|c|c|c|c|c|c|c|c|c|}
\hline \multirow{2}{*}{$\begin{array}{l}\text { Distance } \\
\text { in deg. }\end{array}$} & \multicolumn{8}{|c|}{ Depth of focus in $\mathrm{km}$. } \\
\hline & 100 & 200 & 300 & 400 & 500 & 600 & 700 & 800 \\
\hline 30. & 4 & 5 & 4 & 3 & & & & \\
\hline 40. & 4 & 3 & 3 & 3 & 1 & 0 & & \\
\hline 50. & $\mathbf{5}$ & 5 & 3 & 3 & 1 & 2 & 2 & 1 \\
\hline 60. & 3 & 4 & 3 & 5 & 2 & 2 & 3 & 3 \\
\hline $70 \ldots \ldots \ldots$ & 5 & 5 & 4 & 5 & $\mathbf{5}$ & 3 & 3 & 3 \\
\hline $80 .$. & 4 & 4 & 5 & 5 & 4 & 4 & $\mathbf{5}$ & 4 \\
\hline $90 .$. & 5 & 5 & 4 & 5 & 4 & 4 & 4 & 3 \\
\hline $100 \ldots \ldots \ldots$ & 4 & 5 & 4 & 5 & 4 & 4 & 3 & 3 \\
\hline $110 \ldots \ldots \ldots$ & 4 & 4 & 4 & 4 & 4 & 4 & 4 & 4 \\
\hline \multicolumn{9}{|c|}{$\mathrm{P}^{\prime}$} \\
\hline 145. & 4 & 4 & 4 & 3 & 3 & 4 & 4 & 4 \\
\hline 180. & 4 & 4 & 4 & 4 & 4 & 4 & 4 & 4 \\
\hline
\end{tabular}

TABLE 47

Calculation of the Origin Time by Using $(\mathrm{pP}+\mathrm{P}) / 2$, Shock B (Adgust 29, 1933, South America, Depth of Focus About $650 \mathrm{Km}$.)

(The origin time is $14^{\mathrm{h}} 52^{\mathrm{m}}$ plus $s$ seconds; average, $36^{\mathrm{a}} \pm 4^{\mathrm{s}}$.)

\begin{tabular}{|c|c|c|c|c|c|}
\hline Station & $\begin{array}{l}\text { Distance } \\
\text { in dg. }\end{array}$ & $\begin{array}{c}s \\
\text { sec. }\end{array}$ & Station & $\begin{array}{l}\text { Distance } \\
\text { in deg. }\end{array}$ & $\begin{array}{c}s \\
\text { sec. }\end{array}$ \\
\hline Charlottesville......... & 49.5 & 32 & Ivigtut.... & 73.9 & 32 \\
\hline Georgetown....... & 50.1 & 34 & Victoria............. & 75.9 & 40 \\
\hline Pittsburgh......... & 52.1 & 35 & Malaga ............... & 77.3 & 39 \\
\hline St. Louis. . . & 53.0 & 35 & Cartuja............ & 78.0 & 43 \\
\hline Harvard..... & 53.3 & 35 & Almeria......... & 78.8 & 41 \\
\hline Florissant. . & 53.3 & 32 & Toledo... & 78.9 & 37 \\
\hline Ottawa...... & 56.4 & 36 & Alicante........... & 80.8 & 41 \\
\hline La Jolla....... & 63.1 & 33 & Uccle.... & 88.5 & 36 \\
\hline Riverside.......... & 63.9 & 31 & De Bilt $\therefore \ldots \ldots \ldots \ldots \ldots$ & 89.4 & 36 \\
\hline Pasadena.......... & 64.4 & 30 & Strasbourg............ & 89.9 & 38 \\
\hline Mount Wilson...... & 64.4 & 32 & Stuttgart.............. & 90.8 & 36 \\
\hline Berkeley........... & 69.3 & 31 & & & \\
\hline
\end{tabular}


TABLE 48

Depth of Focus in Km. and Time Intervals sP-P and sS-S in Min.:Sec. For Given Time Interval $\mathrm{pP}-\mathbf{P}$ at Vartous Distances

\begin{tabular}{|c|c|c|c|c|c|c|c|c|c|c|}
\hline \multirow{3}{*}{$\mathrm{pP}-\mathrm{P}$} & \multicolumn{10}{|c|}{ Distance in degrees } \\
\hline & 30 & 40 & 50 & 60 & 70 & 80 & 90 & 100 & 142 & 180 \\
\hline & \multicolumn{8}{|c|}{ Depth of focus, $\mathrm{sP}-\mathrm{P}$ and $\mathrm{sS}-\mathrm{S}$} & \multicolumn{2}{|c|}{$\mathrm{sP}^{\prime}-\mathrm{P}^{\prime}$} \\
\hline $0: 20$ & $\begin{array}{c}100 \\
0: 33 \\
0: 39\end{array}$ & $\begin{array}{c}95 \\
0: 33 \\
0: 38\end{array}$ & $\begin{array}{c}85 \\
0: 33 \\
0: 38\end{array}$ & $\begin{array}{c}80 \\
0: 30 \\
0: 37\end{array}$ & $\begin{array}{c}75 \\
0: 28 \\
0: 36\end{array}$ & $\begin{array}{c}75 \\
0: 29 \\
0: 35\end{array}$ & $\begin{array}{c}75 \\
0: 29 \\
0: 36\end{array}$ & $\begin{array}{c}75 \\
0: 29 \\
0: 38\end{array}$ & $\begin{array}{c}70 \\
0: 26 \\
\ldots\end{array}$ & $\begin{array}{c}65 \\
0: 23 \\
\ldots\end{array}$ \\
\hline $0: 25$ & $\begin{array}{l}125 \\
0: 41 \\
0: 47\end{array}$ & $\begin{array}{l}120 \\
0: 41 \\
0: 47\end{array}$ & $\begin{array}{c}110 \\
0: 39 \\
0: 47\end{array}$ & $\begin{array}{c}100 \\
0: 36 \\
0: 45\end{array}$ & $\begin{array}{c}100 \\
0: 36 \\
0: 44\end{array}$ & $\begin{array}{c}95 \\
0: 36 \\
0: 43\end{array}$ & $\begin{array}{c}95 \\
0: 36 \\
0: 45\end{array}$ & $\begin{array}{c}95 \\
0: 36 \\
0: 47\end{array}$ & $\begin{array}{c}90 \\
0: 34 \\
\ldots\end{array}$ & $\begin{array}{c}85 \\
0: 30\end{array}$ \\
\hline $0: 30$ & $\begin{array}{c}150 \\
0: 48 \\
0: 56\end{array}$ & $\begin{array}{c}145 \\
0: 49 \\
0: 57\end{array}$ & $\begin{array}{c}135 \\
0: 46 \\
0: 57\end{array}$ & $\begin{array}{c}125 \\
0: 43 \\
0: 53\end{array}$ & $\begin{array}{c}120 \\
0: 43 \\
0: 52\end{array}$ & $\begin{array}{c}115 \\
0: 43 \\
0: 51\end{array}$ & $\begin{array}{c}115 \\
0: 42 \\
0: 53\end{array}$ & $\begin{array}{c}115 \\
0: 42 \\
0: 54\end{array}$ & $\begin{array}{c}110 \\
0: 41 \\
\ldots\end{array}$ & $\begin{array}{c}100 \\
0: 38\end{array}$ \\
\hline $0: 35$ & $\begin{array}{c}180 \\
0: 56 \\
1: 04\end{array}$ & $\begin{array}{c}170 \\
0: 57 \\
1: 07\end{array}$ & $\begin{array}{c}160 \\
0: 54 \\
1: 06\end{array}$ & $\begin{array}{c}145 \\
0: 49 \\
1: 01\end{array}$ & $\begin{array}{l}140 \\
0: 50 \\
1: 00\end{array}$ & $\begin{array}{l}140 \\
0: 50 \\
0: 59\end{array}$ & $\begin{array}{l}140 \\
0: 50 \\
1: 01\end{array}$ & $\begin{array}{l}140 \\
0: 50 \\
1: 04\end{array}$ & $\begin{array}{c}130 \\
0: 48\end{array}$ & $\begin{array}{c}120 \\
0: 46 \\
\ldots\end{array}$ \\
\hline $0: 40$ & $\begin{array}{c}205 \\
1: 03 \\
1: 11\end{array}$ & $\begin{array}{c}195 \\
1: 05 \\
1: 16\end{array}$ & $\begin{array}{c}185 \\
1: 02 \\
1: 15\end{array}$ & $\begin{array}{c}165 \\
0: 56 \\
1: 10\end{array}$ & $\begin{array}{c}160 \\
0: 57 \\
1: 08\end{array}$ & $\begin{array}{c}160 \\
0: 57 \\
1: 08\end{array}$ & $\begin{array}{l}160 \\
0: 57 \\
1: 10\end{array}$ & $\begin{array}{l}160 \\
0: 57 \\
1: 12\end{array}$ & $\begin{array}{c}150 \\
0: 55 \\
\ldots\end{array}$ & $\begin{array}{c}140 \\
0: 52\end{array}$ \\
\hline $0: 45$ & $\begin{array}{c}230 \\
1: 11 \\
1: 19\end{array}$ & $\begin{array}{c}220 \\
1: 12 \\
1: 24\end{array}$ & $\begin{array}{l}210 \\
1: 11 \\
1: 24\end{array}$ & $\begin{array}{c}190 \\
1: 04 \\
1: 19\end{array}$ & $\begin{array}{c}180 \\
1: 05 \\
1: 17\end{array}$ & $\begin{array}{c}180 \\
1: 05 \\
1: 17\end{array}$ & $\begin{array}{c}180 \\
1: 05 \\
1: 19\end{array}$ & $\begin{array}{c}180 \\
1: 05 \\
1: 21\end{array}$ & $\begin{array}{c}175 \\
1: 03 \\
\ldots\end{array}$ & $\begin{array}{c}160 \\
1: 00\end{array}$ \\
\hline $0: 50$ & $\begin{array}{c}255 \\
1: 18 \\
1: 26\end{array}$ & $\begin{array}{l}245 \\
1: 19 \\
1: 32\end{array}$ & $\begin{array}{c}235 \\
1: 17 \\
1: 33\end{array}$ & $\begin{array}{l}215 \\
1: 12 \\
1: 27\end{array}$ & $\begin{array}{c}205 \\
1: 13 \\
1: 25\end{array}$ & $\begin{array}{l}205 \\
1: 13 \\
1: 25\end{array}$ & $\begin{array}{l}205 \\
1: 13 \\
1: 28\end{array}$ & $\begin{array}{c}205 \\
1: 13 \\
1: 30\end{array}$ & $\begin{array}{c}195 \\
1: 11 \\
\ldots\end{array}$ & $\begin{array}{c}185 \\
1: 07 \\
\ldots\end{array}$ \\
\hline $0: 55$ & $\begin{array}{c}280 \\
1: 26 \\
1: 33\end{array}$ & $\begin{array}{c}270 \\
1: 27 \\
1: 39\end{array}$ & $\begin{array}{c}260 \\
1: 25 \\
1: 42\end{array}$ & $\begin{array}{l}240 \\
1: 20 \\
1: 36\end{array}$ & $\begin{array}{c}230 \\
1: 20 \\
1: 33\end{array}$ & $\begin{array}{l}225 \\
1: 20 \\
1: 33\end{array}$ & $\begin{array}{l}225 \\
1: 20 \\
1: 36\end{array}$ & $\begin{array}{c}225 \\
1: 20 \\
1: 38\end{array}$ & $\begin{array}{c}215 \\
1: 18 \\
\ldots\end{array}$ & $\begin{array}{c}205 \\
1: 14 \\
\ldots\end{array}$ \\
\hline $1: 00$ & $\begin{array}{c}310 \\
1: 34 \\
1: 40\end{array}$ & $\begin{array}{c}300 \\
1: 35 \\
1: 46\end{array}$ & $\begin{array}{c}290 \\
1: 33 \\
1: 51\end{array}$ & $\begin{array}{c}265 \\
1: 27 \\
1: 45\end{array}$ & $\begin{array}{c}255 \\
1: 27 \\
1: 42\end{array}$ & $\begin{array}{c}250 \\
1: 28 \\
1: 42\end{array}$ & $\begin{array}{c}250 \\
1: 28 \\
1: 45\end{array}$ & $\begin{array}{c}250 \\
1: 28 \\
1: 48\end{array}$ & $\begin{array}{l}235 \\
1: 25\end{array}$ & $\begin{array}{l}225 \\
1: 21\end{array}$ \\
\hline $1: 05$ & $\begin{array}{c}345 \\
1: 43 \\
1: 47\end{array}$ & $\begin{array}{c}330 \\
1: 43 \\
1: 56\end{array}$ & $\begin{array}{l}315 \\
1: 41 \\
1: 59\end{array}$ & $\begin{array}{l}290 \\
1: 35 \\
1: 54\end{array}$ & $\begin{array}{c}275 \\
1: 35 \\
1: 51\end{array}$ & $\begin{array}{c}270 \\
1: 35 \\
1: 50\end{array}$ & $\begin{array}{l}270 \\
1: 35 \\
1: 53\end{array}$ & $\begin{array}{c}270 \\
1: 35 \\
1: 56\end{array}$ & $\begin{array}{c}255 \\
1: 33 \\
\ldots\end{array}$ & $\begin{array}{c}245 \\
1: 28 \\
\ldots\end{array}$ \\
\hline $1: 10$ & $\begin{array}{l}385 \\
1: 53 \\
1: 54\end{array}$ & $\begin{array}{l}365 \\
1: 53 \\
2: 06\end{array}$ & $\begin{array}{c}345 \\
1: 49 \\
2: 08\end{array}$ & $\begin{array}{c}320 \\
1: 43 \\
2: 04\end{array}$ & $\begin{array}{c}300 \\
1: 43 \\
2: 00\end{array}$ & $\begin{array}{l}295 \\
1: 43 \\
1: 58\end{array}$ & $\begin{array}{l}295 \\
1: 42 \\
2: 01\end{array}$ & $\begin{array}{c}295 \\
1: 42 \\
2: 04\end{array}$ & $\begin{array}{c}280 \\
1: 40 \\
\ldots\end{array}$ & $\begin{array}{c}265 \\
1: 36\end{array}$ \\
\hline $1: 15$ & $\begin{array}{c}425 \\
2: 03 \\
2: 00\end{array}$ & $\begin{array}{c}405 \\
2: 03 \\
2: 17\end{array}$ & $\begin{array}{c}375 \\
1: 57 \\
2: 17\end{array}$ & $\begin{array}{l}350 \\
1: 51 \\
2: 14\end{array}$ & $\begin{array}{c}330 \\
1: 51 \\
2: 10\end{array}$ & $\begin{array}{l}320 \\
1: 51 \\
2: 07\end{array}$ & $\begin{array}{c}315 \\
1: 50 \\
2: 10\end{array}$ & $\begin{array}{c}315 \\
1: 50 \\
2: 14\end{array}$ & $\begin{array}{c}300 \\
1: 47\end{array}$ & $\begin{array}{c}290 \\
1: 43\end{array}$ \\
\hline $1: 20$ & & $\begin{array}{c}445 \\
2: 13 \\
2: 28\end{array}$ & $\begin{array}{c}410 \\
2: 05 \\
2: 26\end{array}$ & $\begin{array}{c}380 \\
1: 59 \\
2: 23\end{array}$ & $\begin{array}{c}355 \\
1: 58 \\
2: 19\end{array}$ & $\begin{array}{l}345 \\
1: 58 \\
2: 15\end{array}$ & $\begin{array}{c}340 \\
1: 57 \\
2: 18\end{array}$ & $\begin{array}{l}340 \\
1: 57 \\
2: 23\end{array}$ & $\begin{array}{c}325 \\
1: 55 \\
\ldots\end{array}$ & $\begin{array}{c}310 \\
1: 50 \\
\ldots\end{array}$ \\
\hline
\end{tabular}


TABLE 48--(Concluded)

\begin{tabular}{|c|c|c|c|c|c|c|c|c|c|c|}
\hline \multirow{3}{*}{$\mathrm{pP}-\mathrm{P}$} & \multicolumn{10}{|c|}{ Distance in degrees } \\
\hline & 30 & 40 & 50 & 60 & 70 & 80 & 90 & 100 & 142 & 180 \\
\hline & \multicolumn{8}{|c|}{ Depth of focus, $s P-P$ and $s S-S$} & \multicolumn{2}{|c|}{$\mathbf{s} \mathbf{P}^{\prime}-\mathbf{P}^{\prime}$} \\
\hline $1: 25$ & & $\begin{array}{r}480 \\
2: 23 \\
2: 38\end{array}$ & $\begin{array}{c}440 \\
2: 14 \\
2: 35\end{array}$ & $\begin{array}{c}410 \\
2: 07 \\
2: 32\end{array}$ & $\begin{array}{c}385 \\
2: 06 \\
2: 28\end{array}$ & $\begin{array}{c}370 \\
2: 06 \\
2: 23\end{array}$ & $\begin{array}{c}365 \\
2: 04 \\
2: 26\end{array}$ & $\begin{array}{c}365 \\
2: 04 \\
2: 32\end{array}$ & $\begin{array}{c}350 \\
2: 03 \\
\ldots \ldots\end{array}$ & $\begin{array}{c}335 \\
1: 57 \\
\ldots\end{array}$ \\
\hline $1: 30$ & & $\begin{array}{c}520 \\
2: 32 \\
2: 47\end{array}$ & $\begin{array}{c}470 \\
2: 22 \\
2: 44\end{array}$ & $\begin{array}{l}440 \\
2: 15 \\
2: 41\end{array}$ & $\begin{array}{c}415 \\
2: 14 \\
2: 37\end{array}$ & $\begin{array}{c}395 \\
2: 13 \\
2: 31\end{array}$ & $\begin{array}{c}385 \\
2: 11 \\
2: 35\end{array}$ & $\begin{array}{c}385 \\
2: 11 \\
2: 41\end{array}$ & $\begin{array}{c}375 \\
2: 10 \\
\ldots\end{array}$ & $\begin{array}{c}355 \\
2: 04 \\
\ldots\end{array}$ \\
\hline $1: 35$ & & $\begin{array}{c}560 \\
2: 42 \\
2: 56\end{array}$ & $\begin{array}{c}500 \\
2: 31 \\
2: 53\end{array}$ & $\begin{array}{c}\mathbf{4 7 0} \\
2: 23 \\
2: 49\end{array}$ & $\begin{array}{c}440 \\
2: 22 \\
2: 46\end{array}$ & $\begin{array}{c}420 \\
2: 20 \\
2: 39\end{array}$ & $\begin{array}{c}410 \\
2: 17 \\
2: 44\end{array}$ & $\begin{array}{c}410 \\
2: 17 \\
2: 50\end{array}$ & $\begin{array}{c}395 \\
2: 17 \\
\ldots\end{array}$ & $\begin{array}{c}375 \\
2: 12 \\
\ldots\end{array}$ \\
\hline $1: 40$ & & $\begin{array}{c}605 \\
2: 52 \\
3: 04\end{array}$ & $\begin{array}{c}535 \\
2: 39 \\
3: 02\end{array}$ & $\begin{array}{c}505 \\
2: 32 \\
2: 57\end{array}$ & $\begin{array}{c}465 \\
2: 30 \\
2: 55\end{array}$ & $\begin{array}{c}445 \\
2: 27 \\
2: 48\end{array}$ & $\begin{array}{c}435 \\
2: 24 \\
2: 53\end{array}$ & $\begin{array}{c}435 \\
2: 24 \\
2: 59\end{array}$ & $\begin{array}{c}420 \\
2: 25 \\
\ldots\end{array}$ & $\begin{array}{c}400 \\
2: 19 \\
\ldots\end{array}$ \\
\hline $1: 45$ & & & $\begin{array}{c}570 \\
2: 47 \\
3: 11\end{array}$ & $\begin{array}{c}535 \\
2: 40 \\
3: 06\end{array}$ & $\begin{array}{c}495 \\
2: 38 \\
3: 04\end{array}$ & $\begin{array}{c}475 \\
2: 35 \\
2: 57\end{array}$ & $\begin{array}{c}460 \\
2: 31 \\
3: 02\end{array}$ & $\begin{array}{c}460 \\
2: 31 \\
3: 08\end{array}$ & $\begin{array}{c}450 \\
2: 33 \\
\ldots\end{array}$ & $\begin{array}{c}425 \\
2: 26 \\
\ldots\end{array}$ \\
\hline $1: 50$ & & & $\begin{array}{c}605 \\
2: 55 \\
3: 20\end{array}$ & $\begin{array}{r}570 \\
2: 49 \\
3: 15\end{array}$ & $\begin{array}{c}525 \\
2: 46 \\
3: 14\end{array}$ & $\begin{array}{c}500 \\
2: 42 \\
3: 05\end{array}$ & $\begin{array}{c}485 \\
2: 38 \\
3: 11\end{array}$ & $\begin{array}{c}485 \\
2: 38 \\
3: 17\end{array}$ & $\begin{array}{c}475 \\
2: 41 \\
\ldots\end{array}$ & $\begin{array}{r}445 \\
2: 33 \\
\ldots\end{array}$ \\
\hline $1: 55$ & & & $\begin{array}{r}640 \\
3: 04 \\
3: 29\end{array}$ & $\begin{array}{c}605 \\
2: 58 \\
3: 24\end{array}$ & $\begin{array}{c}560 \\
2: 54 \\
3: 23\end{array}$ & $\begin{array}{c}530 \\
2: 49 \\
3: 14\end{array}$ & $\begin{array}{c}515 \\
2: 45 \\
3: 20\end{array}$ & $\begin{array}{c}515 \\
2: 45 \\
3: 26\end{array}$ & $\begin{array}{c}500 \\
2: 48 \\
\ldots\end{array}$ & $\begin{array}{c}470 \\
2: 40 \\
\ldots\end{array}$ \\
\hline $2: 00$ & & & $\begin{array}{c}675 \\
3: 12 \\
3: 38\end{array}$ & $\begin{array}{c}640 \\
3: 08 \\
3: 34\end{array}$ & $\begin{array}{c}595 \\
3: 03 \\
3: 33\end{array}$ & $\begin{array}{c}565 \\
2: 57 \\
3: 23\end{array}$ & $\begin{array}{c}545 \\
2: 53 \\
3: 29\end{array}$ & $\begin{array}{c}545 \\
2: 53 \\
3: 36\end{array}$ & $\begin{array}{c}525 \\
2: 56 \\
\ldots\end{array}$ & $\begin{array}{c}495 \\
2: 47 \\
\ldots\end{array}$ \\
\hline $2: 05$ & & & $\begin{array}{l}715 \\
3: 21 \\
3: 48\end{array}$ & $\begin{array}{c}680 \\
3: 17 \\
3: 44\end{array}$ & $\begin{array}{c}630 \\
3: 11 \\
3: 42\end{array}$ & $\begin{array}{c}595 \\
3: 05 \\
3: 33\end{array}$ & $\begin{array}{c}570 \\
3: 01 \\
3: 38\end{array}$ & $\begin{array}{c}570 \\
3: 01 \\
3: 45\end{array}$ & $\begin{array}{c}555 \\
3: 03 \\
\ldots\end{array}$ & $\begin{array}{c}515 \\
2: 54 \\
\ldots\end{array}$ \\
\hline $2: 10$ & & & $\begin{array}{l}760 \\
3: 31 \\
3: 58\end{array}$ & $\begin{array}{c}720 \\
3: 26 \\
3: 54\end{array}$ & $\begin{array}{c}665 \\
3: 20 \\
3: 51\end{array}$ & $\begin{array}{c}625 \\
3: 13 \\
3: 42\end{array}$ & $\begin{array}{c}600 \\
3: 09 \\
3: 46\end{array}$ & $\begin{array}{c}600 \\
3: 09 \\
3: 55\end{array}$ & $\begin{array}{c}580 \\
3: 11 \\
\ldots\end{array}$ & $\begin{array}{c}540 \\
3: 01 \\
\ldots\end{array}$ \\
\hline $2: 15$ & & & $\begin{array}{c}805 \\
3: 41 \\
4: 08\end{array}$ & $\begin{array}{c}760 \\
3: 35 \\
4: 04\end{array}$ & $\begin{array}{c}700 \\
3: 28 \\
4: 01\end{array}$ & $\begin{array}{c}655 \\
3: 21 \\
3: 52\end{array}$ & $\begin{array}{c}603 \\
3: 16 \\
3: 55\end{array}$ & $\begin{array}{c}630 \\
3: 16 \\
4: 05\end{array}$ & $\begin{array}{c}610 \\
3: 19 \\
\ldots\end{array}$ & $\begin{array}{c}565 \\
3: 08 \\
\ldots\end{array}$ \\
\hline $2: 20$ & & & & $\begin{array}{c}805 \\
3: 44 \\
4: 14\end{array}$ & $\begin{array}{c}740 \\
3: 37 \\
4: 11\end{array}$ & $\begin{array}{c}690 \\
3: 28 \\
4: 01\end{array}$ & $\begin{array}{c}660 \\
3: 24 \\
4: 04\end{array}$ & $\begin{array}{c}660 \\
3: 24 \\
4: 14\end{array}$ & $\begin{array}{c}635 \\
3: 26 \\
\ldots\end{array}$ & $\begin{array}{c}590 \\
3: 15 \\
\ldots\end{array}$ \\
\hline $2: 25$ & & & & & $\begin{array}{c}780 \\
3: 46 \\
4: 21\end{array}$ & $\begin{array}{c}720 \\
3: 36 \\
4: 10\end{array}$ & $\begin{array}{r}690 \\
3: 32 \\
4: 13\end{array}$ & $\begin{array}{r}690 \\
3: 31 \\
4: 23\end{array}$ & $\begin{array}{c}665 \\
3: 33 \\
\ldots\end{array}$ & $\begin{array}{c}620 \\
3: 23 \\
\ldots\end{array}$ \\
\hline $2: 30$ & & & & & $\begin{array}{l}820 \\
3: 55 \\
4: 30\end{array}$ & $\begin{array}{c}755 \\
3: 44 \\
4: 19\end{array}$ & $\begin{array}{c}720 \\
3: 40 \\
4: 22\end{array}$ & $\begin{array}{c}715 \\
3: 39 \\
4: 32\end{array}$ & $\begin{array}{c}690 \\
3: 40 \\
\therefore\end{array}$ & $\begin{array}{c}645 \\
3: 30 \\
\ldots\end{array}$ \\
\hline $2: 35$ & & & & & & $\begin{array}{l}785 \\
3: 51 \\
4: 27\end{array}$ & $\begin{array}{c}755 \\
3: 48 \\
4: 31\end{array}$ & $\begin{array}{l}745 \\
3: 46 \\
4: 41\end{array}$ & $\begin{array}{c}720 \\
3: 47 \\
\ldots\end{array}$ & $\begin{array}{c}670 \\
3: 38 \\
\ldots\end{array}$ \\
\hline
\end{tabular}


It will be seen from table 46 that if we take $c=4$ sec. the method gives the correct origin time for any unknown depth not exceeding $800 \mathrm{~km}$., with a maximum error of two seconds (except for the greater depths at distances from $40^{\circ}$ to $50^{\circ}$ ). If the depth is known, the proper value of $c$ can be taken from the table, and the origin time can then be found.

TABLE 49

Corrections, in Degrees, for Depth of Focus to be Added to Distances FOUND FROM TABLE 45

\begin{tabular}{|c|c|c|c|c|c|c|c|c|}
\hline \multirow{2}{*}{$\begin{array}{c}\text { Distance } \\
\text { in deg. }\end{array}$} & \multicolumn{8}{|c|}{ Depth of focus in $\mathrm{km}$. } \\
\hline & 100 & 200 & 300 & 400 & 500 & 600 & 700 & 800 \\
\hline $10 .$. & $-1 / 2$ & 0 & 0 & 0 & 0 & 0 & 0 & 0 \\
\hline $15 .$. & 0 & $1 / 2$ & $1 / 2$ & 1 & 1 & $1 / 2$ & $1 / 2$ & $1 / 2$ \\
\hline $20 \ldots \ldots$ & $1 / 2$ & 1 & $11 / 2$ & 2 & 2 & 2 & $11 / 2$ & $11 / 2$ \\
\hline $25 \ldots \ldots \ldots \ldots$ & 1 & $11 / 2$ & $21 / 2$ & $3 \frac{1}{2}$ & 4 & $41 / 2$ & $51 / 2$ & $61 / 2$ \\
\hline $30 \ldots$ & $11 / 2$ & 2 & 3 & 4 & 5 & 6 & 7 & $81 / 2$ \\
\hline $35 \ldots \ldots \ldots$ & 1 & 2 & 3 & 4 & $51 / 2$ & $61 / 2$ & $71 / 2$ & 9 \\
\hline $40 \ldots$ & 1 & 2 & 3 & $41 / 2$ & $51 / 2$ & $61 / 2$ & $71 / 2$ & $81 / 2$ \\
\hline $45 \ldots \ldots$ & 1 & $21 / 2$ & $31 / 2$ & $4 \frac{1}{2}$ & $51 / 2$ & $61 / 2$ & $71 / 2$ & $81 / 2$ \\
\hline 50. & $11 / 2$ & 3 & 4 & 5 & 6 & 7 & 8 & 9 \\
\hline 55. & $1 \frac{1}{2}$ & 3 & $41 / 2$ & $51 / 2$ & $61 / 2$ & 8 & 9 & 10 \\
\hline $60 .$. & $1 \frac{1}{2}$ & 3 & $41 / 2$ & 6 & 7 & $81 / 2$ & 10 & 11 \\
\hline$\ldots \ldots \ldots$ & $1 \frac{1}{2}$ & 3 & $41 / 2$ & 6 & $71 / 2$ & $81 / 2$ & 10 & 11 \\
\hline $70 \ldots \ldots$ & $1 \frac{1}{2}$ & 3 & $41 / 2$ & 6 & 7 & 8 & $91 / 2$ & 11 \\
\hline $75 \ldots$ & $1 / 2$ & 3 & $41 / 2$ & $51 / 2$ & $61 / 2$ & 8 & $91 / 2$ & $11 \frac{1 / 2}{2}$ \\
\hline $80 .$. & $11 / 2$ & 3 & $41 / 2$ & 6 & 7 & 9 & $101 / 2$ & 13 \\
\hline $85 .$. & 2 & $31 / 2$ & 5 & 7 & $81 / 2$ & $101 / 2$ & $12 \frac{1}{2}$ & 14 \\
\hline $90 \ldots$ & 2 & $31 / 2$ & $51 / 2$ & 8 & 10 & 12 & $131 / 2$ & 15 \\
\hline $95 \ldots \ldots$ & 2 & 4 & 6 & $81 / 2$ & $101 / 2$ & $121 / 2$ & 14 & $15 \frac{1}{2}$ \\
\hline $100 \ldots \ldots$ & 2 & 4 & 6 & $81 / 2$ & $101 / 2$ & $12 \frac{1}{2}$ & 14 & $15 \frac{1}{2}$ \\
\hline
\end{tabular}

An example of this method is given in table 47. The deviations from the mean are rather small although this is an unfavorable instance, since the epicenter could not be determined very precisely and the depth was unusually large.

The use of $\mathrm{P}^{\prime} \mathrm{P}^{\prime}$, PKKP, etc., to determine the origin time is discussed in the two sections next following.

\section{Calculation of the Depth}

There are two general methods for determining focal depth: (a) from the intervals between recorded phases (the origin time need not be known, and the distance is required only to a rough approximation); and (b) from the travel times of various phases, when the distance and origin time have been determined. 
(a) This is the natural procedure when the data of only one station are available; but since it requires that the phases shall be correctly identified, there is a frequent risk of large errors, which can only be avoided when the observations of several stations are used.

The most important interval for this purpose is $\mathrm{pP}-\mathrm{P}$ (or $\mathrm{pP}^{\prime}-\mathrm{P}^{\prime}$ ). Table 48 gives the depth corresponding to given values of this interval at various distances. The intervals $\mathrm{sP}-\mathrm{P}$ and $\mathrm{sS}-\mathrm{S}$, given in the same table, are available either as a check or independently.

The use of the interval $\mathrm{P}^{\prime} \mathrm{P}^{\prime}-\mathrm{P}^{7}$ or PKKP $-\mathrm{P}$ ("On Seismic Waves, I," $\mathrm{p}$. 116) presents certain special features. These intervals vary only slowly with depth, but rapidly with distance; so that the approximate distance of the shock, without regard to focal depth, can be found from the data tables 35 and 33 , respectively. This comparatively precise determination frequently eliminates uncertainty in the identification of $\mathrm{pP}, \mathrm{sP}$, etc., so that all the observed phases are then available to determine origin time and, eventually, depth.

The use of sScS-ScS has been discussed by Hayes. ${ }^{8}$ (See table 41.)

(b) Some travel times give much more precise determinations of depth than others. This may be seen from table 5 . The difference in time corresponding to a given difference in focal depth is almost twice as large for waves starting as transverse waves as for waves leaving the focus as longitudinal waves. However, it is generally not advisable to use the direct $\mathbf{S}$, as the travel times of this phase still present a number of unsolved problems, and the observations often lack precision because of small amplitudes and indefinite phase beginnings.

Good results can usually be obtained from SKS-O at distances from $90^{\circ}$ to $130^{\circ}$, and from $\mathrm{ScS}-\mathrm{O}$ from $0^{\circ}$ to $50^{\circ}$. Precise results also can of ten be obtained from $\mathrm{sScS}$ in the limited range of distance (up to about $30^{\circ}$ ) for which this phase is observed. ${ }^{9}$

$\mathrm{P}-\mathrm{O}$ is not quite so valuable a datum for the determination of depth as might be expected; for the greater reliability of the observations is compensated by the circumstance that the times are only about half as sensitive to variations in depth as those of the $\mathrm{S}$ group of phases. Depths found from $\mathrm{P}-\mathrm{O}$ usually show more scatter than those determined in the other ways discussed above. $\mathrm{PP}-\mathrm{O}$ gives still less accurate results.

Other phases are occasionally available. Very good results are occasionally obtained from sP and SP, which are theoretically very favorable for the application of the method since their times have the sensitivity to depth which is characteristic of transverse waves, and since they are observed with the precision of longitudinal waves.

${ }^{7}$ B. Gutenberg and C. F. Richter, "On P'P' and Related Phases," Gerlands Beitr. $z$. Geophys., 41:149-159 (134).

${ }^{8}$ R. C. Hayes, "A New Phase in Deep-Focus Earthquakes." Dominion Observatory Wellington Bull. no. 107 (1936).

${ }^{9}$ Hayes, op. cit. 


\section{Interpretation of a Single Seismogram}

In undertaking the interpretation of a single seismogram of a deep-focus earthquake, the following order of procedure has been found useful at Pasadena, and is adhered to as far as the data are available:

(1) The distance is determined from $\mathrm{P}^{\prime} \mathrm{P}^{\prime}-\mathrm{P}$ (table 35 ) or $\mathrm{PKKP}-\mathrm{P}$ (table 33).

(2) $\mathrm{O}$ is found by using $\mathrm{S}-\mathrm{P}$ to derive $\mathrm{P}-\mathrm{O}$ (table 45). From the same data is determined $\Delta_{25}$, the distance corresponding to a normal shock with the same $\mathrm{P}-\mathrm{O}$.

(3) The depth is found from $\mathrm{P}^{\prime} \mathrm{P}^{\prime}-\mathrm{O}$ or $\mathrm{PKKP}-\mathrm{O}$ (tables 35 and 33 ).

(4) As distance, origin time, and depth are now known to a first approximation, the identification of phases can be verified, and the depth can be determined independently from $\mathrm{pP}-\mathrm{P}, \mathrm{sP}-\mathrm{P}, \mathrm{sS}-\mathrm{S}$, etc.

(5) A corrected distance can be found by applying to $\Delta_{25}$ the correction given in table 49. (This table replaces the data given as figure 2 of our previous paper. Note that this figure was derived from Jeffreys' tables for normal shocks, whereas the present table 49 has been calculated from the travel times given in table 45.)

(6) A final check is now available by comparing all recorded phases with a travel-time curve constructed for the proper depth, similar to figure 1 (the folded graph following p. 390).

TABLE 50

LIST OF SHOCKs

\begin{tabular}{|c|c|c|c|c|c|c|}
\hline \multirow{2}{*}{ Sh. } & \multirow{2}{*}{ Date } & \multirow{2}{*}{ Region } & \multirow{2}{*}{ Latitude } & \multirow{2}{*}{ Longitude } & \multicolumn{2}{|c|}{ Origin time from } \\
\hline & & & & & $S-P$ & $(\mathrm{pP}+\mathrm{P}) / 2$ \\
\hline A & 1931, Feb. 20. & E. Siberia & $44^{\circ} \cdot 3 \mathrm{~N}$ & $135^{\circ} .5 \mathrm{E}$ & $5^{\mathrm{h}} 33^{\mathrm{m}} 21^{\mathrm{a}} \pm 5^{\mathrm{s}} .5$ & $24^{s} \pm 2^{s}$ \\
\hline $\mathrm{B}$ & 1933, Aug. 29. & S. America & $10^{\circ} 56^{\prime} \mathrm{S}, \pm 29^{\prime}$ & $69^{\circ} 28^{\prime} \mathrm{W}, \pm 1^{\circ} 24^{\prime}$ & $14^{\mathrm{h}} 52^{\mathrm{m}} 33^{\mathrm{s}} \pm 6^{\mathrm{s}}$ & $36^{3} \pm 4^{\mathrm{s}}$ \\
\hline $\mathrm{C}$ & 1932, Jan. 9 & Solomon Is. & $6^{\circ} 10^{\prime} \mathrm{S}, \pm 7^{\prime}$ & $154^{\circ} 30^{\prime} \mathbf{E}, \pm 39^{\prime}$ & $10^{\mathrm{h}} 21^{\mathrm{m}} 45^{\mathrm{s}} \pm 5^{\mathrm{s}} \cdot 5$ & $42^{\mathrm{s}} \pm 2^{\mathrm{s}}$ \\
\hline $\mathrm{D}$ & 1934, Jan. $3 \ldots$ & Kamchatka & $52^{\circ} 50^{\prime} \mathrm{N}, \pm 9^{\prime}$ & $156^{\circ} 39^{\prime} \mathbf{E}, \pm 28^{\prime}$ & $9^{\mathrm{h}} 42^{\mathrm{m}} 24^{\mathrm{s}} \pm 6^{\mathrm{s}}$ & $27^{\mathrm{s}} \pm 3.5$ \\
\hline $\mathbf{E}$ & 1928, Mar. 29. & S. of Japan & $31^{\circ} 45^{\prime} \mathrm{N}$ & $138^{\circ} 12^{\prime} \mathrm{E}$ & $5^{\mathrm{h}} 06^{\mathrm{m}} 02^{\mathrm{s}} \pm 5^{\mathrm{s}} 5$ & $03^{8} \pm 2^{\$ 5}$ \\
\hline $\mathbf{F}$ & 1933, Sept. 2.. & S. of Japan & $29^{\circ} 05^{\prime} \mathrm{N}, \pm 23^{\prime}$ & $138^{\circ} 46^{\prime} \mathrm{E}, \pm 34^{\prime}$ & $16^{\mathrm{h}} 41^{\mathrm{m}} 14^{\mathrm{g}} \pm 5^{\mathrm{s}}$ & $08^{8} \pm 1^{8.5}$ \\
\hline $\mathbf{G}$ & 1932, Apr. 4. & S. of Japan & $29^{\circ} 05^{\prime} \mathrm{N}, \pm 23^{\prime}$ & $138^{\circ} 46^{\prime} \mathrm{E}, \pm 34^{\prime}$ & $19^{\mathrm{h}} 16^{\mathrm{m}} 34^{\mathrm{a}} \pm 6^{\mathrm{s}} .5$ & $31^{s} \pm 2^{8}$ \\
\hline H & 1929, Feb. 1. & Hindu Kush & $36^{\circ} .5 \mathrm{~N}$ & $70^{\circ} .5 \mathrm{E}$ & $17^{\mathrm{h}} 14^{\mathrm{m}} 17^{\mathrm{s}} \pm 9^{\mathrm{s}}$ & $261 / 2^{8} \pm 3^{s}$ \\
\hline $\mathbf{I}$ & 1921, Nov. 15 & Hindu Kush & $36.5 \mathrm{~N}$ & $70^{\circ} .5 \mathrm{E}$ & $20^{\mathrm{h}} 36^{\mathrm{m}} 32^{\mathrm{s}} \pm 7^{\mathrm{s}}$ & $381 / 2^{\mathrm{s}} \pm 4^{\mathrm{s} 5}$ \\
\hline$J$ & 1928, Aug. 10. & Hindu Kush & $36^{\circ} .5 \mathrm{~N}$ & $70^{\circ} .5 \mathrm{E}$ & $15^{\mathrm{h}} 33^{\mathrm{m}} 40^{\mathrm{s}} \pm 7^{\mathrm{s}}$ & $\left(48^{\mathrm{s}}\right)$ \\
\hline $\mathrm{K}$ & 1931, Oct. 5 & Hindu Kush & $36^{\circ} .5 \mathrm{~N}$ & $70^{\circ} .5 \mathrm{E}$ & $22^{\mathrm{h}} 31^{\mathrm{m}} 22^{\mathrm{s}} \pm 6^{\mathrm{s}}$ & $27^{s} \pm 3^{s}$ \\
\hline $\mathrm{L}$ & 1933, Jan. 9.. & Hindu Kush & $36.5 \mathrm{~N}$ & $70^{\circ} .5 \mathrm{E}$ & $2^{\mathrm{h}} 01^{\mathrm{m}} 36^{\mathrm{s}} \pm 6^{\mathrm{s}}$ & $43^{s} \pm 2^{s}$ \\
\hline $\mathbf{M}$ & 1934, July 22. & Hindu Kush & $36^{\circ} .5 \mathrm{~N}$ & $70^{\circ} .5 \mathrm{E}$ & $19^{\mathrm{h}} 56^{\mathrm{m}} 49^{\mathrm{s}} \pm 6^{\mathrm{s}}$ & $57^{\mathrm{B}} \pm 3^{\mathrm{B}}$ \\
\hline $\mathrm{N}$ & 1934, Nov. $18 \ldots$ & Hindu Kush & $36.5 \mathrm{~N}$ & $70.5 \mathrm{E}$ & $3^{\mathrm{h}} 21^{\mathrm{m}} 15^{\mathrm{s}} \pm 6^{\mathrm{s}}$ & $24^{\mathrm{a}} \pm 3^{\mathrm{B}}$ \\
\hline 0 & 1935, Dec. $14 \ldots$ & S. America & Near B & Near B & $1^{h}+$ & \\
\hline $\mathrm{P}$ & 1935, Dec. 16. & S. America & Near B & Near B & $16^{\mathrm{h}}+$ & \\
\hline
\end{tabular}

ReMarks-Shock A: epicenter as given by Scrase. Shock E: epicenter as given by Stechschulte. Shock G same epicenter adopted as for shock $\mathrm{F}$ after comparison of travel times; see table $51 b$. Shocks I to $\mathrm{N}$ : same epicenter adopted as for shock $H$; see table $51 a$; the epicenter of shock $H$ has been taken from Jeffreys. For shocks $B, O$, and $\mathrm{P}$, see table 51c. Errors following " \pm " are mean errors. 
TABLE 51

Comparison of Travel Times

(The columns headed by "m" give the minutes, the following columns the additional seconds.)

(a) Hindu Kush shocks ( $\mathrm{H}$ to $\mathrm{N}$ of table 50). Depth of focus, $220 \mathrm{~km}$.

\begin{tabular}{|c|c|c|c|c|c|c|c|c|c|c|c|c|c|c|c|c|c|}
\hline \multirow{2}{*}{ Station } & \multirow{2}{*}{$\begin{array}{l}\text { Distance } \\
\text { in deg. }\end{array}$} & \multicolumn{8}{|c|}{ Travel times of $\mathbf{P}$ in shock } & \multicolumn{8}{|c|}{ Travel times of $\mathrm{S}$ in shock } \\
\hline & & $\mathrm{m}$ & $\mathbf{H}$ & $\mathbf{I}$ & $\mathbf{J}$ & $\mathbf{K}$ & $\mathbf{L}$ & $\mathbf{M}$ & $\mathrm{N}$ & $\mathrm{m}$ & $\mathrm{H}$ & I & $\mathrm{J}$ & $\mathrm{K}$ & $\mathrm{L}$ & $\mathbf{M}$ & $\mathrm{N}$ \\
\hline Bombay.. & 17.7 & 3 & 55 & 47 & 53 & & 54 & 53 & 51 & 8 & 75 & 57 & 67 & & 67 & 56 & 59 \\
\hline Sverdlovsk.. & 21.4 & 4 & 33 & & 31 & 40 & 34 & 32 & 31 & 8 & 09 & & 13 & 22 & 14 & 15 & 11 \\
\hline Helwan. & 332 & 6 & 22 & 19 & & & 23 & 20 & 19 & 11 & 25 & 19 & & & 30 & 31 & 31 \\
\hline Pulkovo.. & 34.6 & 6 & 32 & & 31 & 31 & 30 & 29 & 32 & 11 & 46 & & 43 & 47 & 43 & 43 & 46 \\
\hline Hong Kong. & 40.2 & 7 & & 19 & & 13 & 18 & & 19 & 13 & & 08 & & 06 & 09 & & 04 \\
\hline Zi Ka Wei.. & 42.1 & 7 & & 33 & 28 & 31 & 33 & 31 & & 13 & & 39 & 36 & 29 & 44 & 35 & . \\
\hline Copenhagen. & 43.1 & 7 & 43 & & 41 & 43 & 43 & & 45 & 13 & 54 & & 52 & 55 & 54 & & 60 \\
\hline Hamburg. & 445 & 7 & 55 & 56 & & 56 & 54 & 52 & & 14 & 17 & 17 & & 18 & 16 & & \\
\hline Zürich.... & 46.1 & 8 & 05 & 06 & 05 & 07 & 06 & 02. & 08 & 14 & 38 & 37 & & 35 & 36 & & \\
\hline Strasbourg. & 465 & 8 & 10 & 09 & 09 & & 08 & & 09 & 14 & 43 & 45 & 44 & & 42 & 44 & 50 \\
\hline De Bilt... & 47.6 & 8 & 18 & 19 & 17 & 18 & 18 & 17 & 20 & 14 & 59 & 60 & 58 & 60 & 60 & 59 & 66 \\
\hline Manila... & 49.8 & 8 & 34 & 25 & & 33 & 34 & 23 & 35 & 14 & 28 & & & & 20 & 26 & 27 \\
\hline Tortosa. & 53.4 & 9 & 01 & 03 & 01 & 04 & 02 & & 06 & 16 & 20 & 12 & & 24 & 19 & & 24 \\
\hline Toledo... & 570 & 9 & 25 & & 25 & 27 & 26 & 24 & 27 & 17 & & & & 07 & 02 & 03 & 13 \\
\hline Cartuja. . & 57. 6 & 9 & 29 & 33 & 30 & 31 & 33 & & 34 & 17 & 21 & 16 & 17 & 21 & 11 & & 19 \\
\hline
\end{tabular}

$\mathbf{P}^{\prime}$

\begin{tabular}{l|l|l|l|l|l|l|l|l|l|l|l|l|l|l|l|l|l}
\hline $\mathrm{La}$ Paz................ & 138.4 & 19 & 03 & 01 & & 04 & 04 & 03 & 05 & & & & & & & \\
\hline
\end{tabular}

(b) Shocks south of Japan (F and G of table 50). Depth of focus, $410 \mathrm{~km}$.

\begin{tabular}{|c|c|c|c|c|c|c|c|}
\hline \multirow{3}{*}{ Station } & \multirow{3}{*}{$\begin{array}{l}\text { Distance } \\
\text { in deg. }\end{array}$} & \multicolumn{4}{|c|}{ Travel times in Min.: Sec. of } & \multirow{2}{*}{\multicolumn{2}{|c|}{$\begin{array}{c}\text { Difference } \\
\mathrm{pP}-\mathrm{P}\end{array}$}} \\
\hline & & \multicolumn{2}{|c|}{$\mathbf{P}$} & \multicolumn{2}{|c|}{$\mathbf{S}$} & & \\
\hline & & $\mathrm{F}$ & $\mathrm{G}$ & $\mathbf{F}$ & G & $\mathbf{F}$ & $\mathrm{G}$ \\
\hline 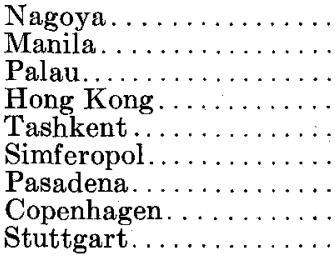 & $\begin{array}{r}6.1 \\
22.0 \\
22.2 \\
23.2 \\
56.6 \\
79.3 \\
83.8 \\
83.3 \\
90.2\end{array}$ & $\begin{array}{r}1: 36 \\
4: 34 \\
4: 40 \\
4: 42 \\
9: 09 \\
11: 27 \\
11: 49 \\
11: 48 \\
12: 19\end{array}$ & $\begin{array}{r}1: 33 \\
4: 35 \\
4: 43 \\
4: 41 \\
9: 07 \\
11: 25 \\
11: 49 \\
11: 46 \\
12: 17\end{array}$ & $\begin{array}{r}2: 42 \\
8: 20 \\
8: 18 \\
8: 26 \\
16: 21 \\
20: 47 \\
21: 29 \\
21: 26 \\
21: 31\end{array}$ & $\begin{array}{r}2: 38 \\
\\
8: 22 \\
8: 23 \\
16: 19 \\
20: 41 \\
21: 34 \\
21: 26\end{array}$ & $\begin{array}{l}1: 33 \\
1: 32 \\
1: 36\end{array}$ & $\begin{array}{l}1: 36 \\
1: 40 \\
1: 41\end{array}$ \\
\hline 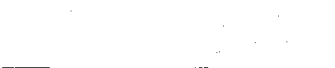 & & & & & & & \\
\hline La Paz . . . . . . . . . & 152.2 & $19: 11$ & $19: 12$ & & & & \\
\hline
\end{tabular}


TABLE 51-(Concluded)

(c) Shocks in South America (B, O, and P of table 50). Depth of focus, about $650 \mathrm{~km}$.

\begin{tabular}{|c|c|c|c|c|c|c|c|c|c|c|}
\hline \multirow{4}{*}{ Station } & \multirow{4}{*}{$\begin{array}{c}\text { Dis- } \\
\text { tance in } \\
\text { deg. } \\
\mathbf{B}\end{array}$} & \multicolumn{6}{|c|}{ Difference in time of arrival } & \multirow{2}{*}{\multicolumn{3}{|c|}{$\frac{\mathrm{pP}-\mathrm{P} \text { in Min.: Sec. }}{\text { Shock }}$}} \\
\hline & & \multirow{2}{*}{\multicolumn{3}{|c|}{$\frac{\text { Shock } O-B}{36^{\mathrm{d}} 10^{\mathrm{h}} 38^{\mathrm{m}} \text { plus sec.: }}$}} & \multirow{2}{*}{\multicolumn{3}{|c|}{$\frac{\text { Shock } P-O}{2^{d} 15^{\text {h }} 26^{\mathrm{m}} \text { plus sec.: }}$}} & & & \\
\hline & & & & & & & & \multirow{2}{*}{ B } & \multirow{2}{*}{0} & \multirow[b]{2}{*}{$\mathbf{P}$} \\
\hline & & $\mathbf{P}$ & $\mathrm{pP}$ & s & $P$ & $\mathrm{pP}$ & $\mathbf{s}$ & & & \\
\hline 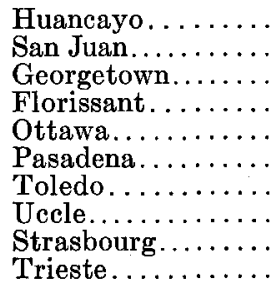 & $\begin{array}{l}5.8 \\
29.4 \\
50.1 \\
53.3 \\
56.4 \\
64.4 \\
78.8 \\
88.4 \\
89.9 \\
93.1\end{array}$ & $\begin{array}{l}39 \\
24 \\
31 \\
23 \\
26 \\
28 \\
34 \\
36 \\
25\end{array}$ & $\begin{array}{l}33 \\
37 \\
\\
35 \\
34\end{array}$ & $\begin{array}{l}37 \\
\\
22 \\
09 \\
16 \\
21 \\
39 \\
\\
\\
36\end{array}$ & $\begin{array}{l}11 \\
08 \\
10 \\
07 \\
07 \\
15 \\
09\end{array}$ & 02 & $\begin{array}{l}13 \\
08 \\
11 \\
01\end{array}$ & $\begin{array}{l}1: 54 \\
1: 56 \\
1: 58 \\
2: 03 \\
2: 08 \\
2: 16 \\
2: 09\end{array}$ & $\begin{array}{l}1: 56 \\
2: 12 \\
\\
2: 15 \\
2: 18\end{array}$ & $2: 04$ \\
\hline \multicolumn{11}{|c|}{$\mathrm{P}^{\prime}$} \\
\hline $\begin{array}{l}\text { Bombay....... } \\
\text { Nagoya........ } \\
\text { Chiufeng...... } \\
\text { Manila....... }\end{array}$ & $\begin{array}{l}142.7 \\
146.0 \\
150.6 \\
169.2\end{array}$ & $\begin{array}{l}32 \\
34 \\
37\end{array}$ & & & $\begin{array}{l}12 \\
10 \\
11\end{array}$ & & & & & \\
\hline
\end{tabular}

This procedure applies more particularly to deep-focus shocks at distances not exceeding about $120^{\circ}$. Large deep-focus shocks at greater distances are rarely recorded at Pasadena and are studied by the direct use of the proper travel-time curves.

\section{Application of the Methods to Selected Shocks}

Table 50 lists the shocks which have been selected for testing the data and methods of the preceding sections. The epicenters of shocks B, C, D, and F have been found by the method described on pages $373 \mathrm{ff}$. The mean errors of coördinates (and origin times) are given in the table. For shock A we have used the epicenter determined by Scrase; $;^{10}$ for shock E, that found by Stechschulte; ${ }^{11}$ and for shock $\mathrm{H}$, the epicenter as given in the International Seismological Summary (this shock has been specially studied by Jeffreys ${ }^{2}$ ).

It was found that the travel times of shock $G$ agree with those of shock $F$ within the limits of error. Selected data for $\mathrm{P}, \mathrm{S}$, and $\mathrm{pP}-\mathrm{P}$ are given in table

${ }^{10}$ F. J. Scrase, "The Characteristics of a Deep-Focus Earthquake," Philos. Trans. Roy. Soc: London, (A) 231:207-234 (1933).

${ }^{11}$ V. C. Stechschulte, "The Japanese Earthquake of March 29, 1928, and the Problem of Depth of Focus," Bull. Seism. Soc. Am., 22:81-137 (1932).

${ }_{12}$ H. Jeffreys, "Some Deep-Focus Earthquakes," Monthly Not. Roy. Astron. Soc., Geophys. Suppl., 3:310-343 (1935). 
$51 b$. These are quite representative of the data of the better stations; the same epicenter consequently has been used for both shocks.

Similar remarks apply to the South American shocks B, O, and P; the two last named have not yet been investigated in detail, but the available data, of which table 51c gives an excerpt, indicate that the foci cannot have been far apart. Shock $P$ agrees particularly well with shock $O$, of which it must be considered to have been an aftershock.

A rather large number of shocks can be found in the International Summary and the reports of the Russian group of stations, which originate in the Hindu Kush region and exhibit deep-focus characteristics. The reported times of a number of these have been compared by the method of differences with those of shock $\mathrm{H}$; a part of the data for certain of the better stations which report a majority of these shocks is reproduced in table 51a. It will be seen that it is clearly justifiable to refer the entire group of shocks, $\mathrm{H}$ to $\mathrm{N}$ inclusive, to a common focus, within small limits of error.

The largest shock of this group is shock I; owing to the early date, the times are not as dependable as those for the later shocks. Nevertheless, the agreement is very good. Certain seismograms of this shock have been published; ${ }^{13}$ these have been carefully examined for the present study. Shock $\mathrm{L}$ is the largest of the later shocks of this group.

The origin times of these shocks have been determined from $\mathrm{S}-\mathrm{P}$ and from $\frac{(\mathrm{pP}+\mathrm{P})}{2}$, as described above (p. 374); the results are given in the last columns of table 50. From 20 to 40 stations were available in the several shocks. The mean error in seconds is attached to each time. These errors for the second method are considerably smaller than those from $\mathrm{S}-\mathrm{P}$, indicating a smaller scattering of the results; this is to be expected from the nature of the observations of the $\mathbf{S}$ phase. However, the smaller scattering from the second method does not exclude the possibility of systematic error. This may be examined by comparing the results of the two methods, which in general are consistent. For the Hindu Kush shocks, a large number of the better stations are at distances of from $45^{\circ}$ to $55^{\circ}$, where the beginning of $S$ is frequently indefinite; this results in late readings for $\mathbf{S}$ and consequent early origin times. On the other hand, origin times determined from $\mathrm{P}$ and $\mathrm{pP}$ are more likely to be late, owing to late readings of one or both phases. Where the times determined by the two methods agree, as here, it is very probable that the result is unaffected by systematic error.

The origin times given in the last column of table 50 have been adopted for use in further calculation and study.

Calculated focal depths are given in table 52. The first three columns refer to

${ }^{13}$ Mitteilungen der deutschen Erdbebenwarten über die Aufzeichnungen des Bebens vom 15. Nov. 1921, Tafeln XVI-XIX (Hauptstation für Erdbebenforschung in Jena 1922). See also two seismograms in Handbuch d. Geophysik, 4:194, fig. 87. 
depths found from time differences between the phases; these are independent of the origin time, and depend very little on the epicenter. The very good agreement between these results and those of the three following columns, which depend on the origin time, indicates that the origin times used cannot be far wrong. The general agreement among all the results shows that there can be no large errors in the curves used. The mean error is attached to each calculated

TABLE 52

Calculated Depth of Focus

\begin{tabular}{|c|c|c|c|c|c|c|c|c|}
\hline \multirow{2}{*}{$\begin{array}{l}\text { Date of } \\
\text { shock }\end{array}$} & \multirow{2}{*}{$\begin{array}{l}\text { Region of } \\
\text { shoek }\end{array}$} & \multicolumn{6}{|c|}{ Depth in $\mathrm{km}$. calculated from } & \multirow{2}{*}{ Remarks } \\
\hline & & $\mathbf{p P}-\mathbf{P}$ & $\mathrm{sP}-\mathrm{P}$ & $s S-S$ & $\mathrm{P}-\mathrm{O}$ & SKS-O & $\mathrm{SeS}-\mathrm{O}$ & \\
\hline 1931, Feb. 20 & E. Siberia..... & $350 \pm 20$ & $360 \pm 25$ & $365 \pm 15$ & $350 \pm 25$ & $350 \pm 25$ & 320 & Scrase 360 \\
\hline 1933, Aug. 29 & S. America... & $640 \pm 15$ & & & $660 \pm 60$ & $650 \pm 35$ & $670 \pm 50$ & $\mathrm{P}^{\prime}-\mathrm{O} 620 \pm 55$ \\
\hline 1932, Jan. 9 & Solomon Is.. & $400 \pm 20$ & & $400 \pm 35$ & $375 \pm 25$ & $380 \pm 35$ & & $\mathrm{pP}^{\prime}-\mathrm{P}^{\prime} 360 \pm 30$ \\
\hline 1934, Jan. 3 & Kamohatka.. & $280 \pm 20$ & $300 \pm 20$ & $300 \pm 20$ & $280 \pm 20$ & $280 \pm 40$ & $260 \pm 30$ & \\
\hline 1928, Mar. 29 & S. of Japan... & $420 \pm 15$ & & $410 \pm 10$ & $410 \pm 35$ & $410 \pm 20$ & $410 \pm 35$ & Stechschulte 410 \\
\hline 1933, Sept. 2 & S. of Japan.... & $410 \pm 10$ & & $430 \pm 70$ & $400 \pm 60$ & $420 \pm 20$ & $390 \pm 20$ & Only 4 sS used \\
\hline 1932, Apr. 4 & S. of Japan.. & $430 \pm 15$ & & & $400 \pm 45$ & $420 \pm 15$ & & \\
\hline 1929, Feb. 1 & Hindu Kush. & $240 \pm 20$ & $235 \pm 15$ & $210 \pm 15$ & $220 \pm 30$ & $215 \pm 20$ & & Jeffireys 212 \\
\hline 1921, Nov. 15 & Hindu Kush. . & $230 \pm 15$ & & $200 \pm 15$ & $220 \pm 30$ & $220 \pm 25$ & $200 \pm 20$ & \\
\hline 1928, Aug. 10 & Hindu Kush. & & $230 \pm 5$ & & $230 \pm 35$ & & & \\
\hline 1931, Oct. 5 & Hindu Kush. . & $230 \pm 20$ & $230 \pm 20$ & $210 \pm 30$ & $220 \pm 30$ & 210 & & Only 3 SKS \\
\hline 1933, Jan. 9 & Hindu Kush. . & $240 \pm 30$ & $230 \pm 20$ & $210 \pm 20$ & $230 \pm 30$ & $220 \pm 15$ & $210 \pm 15$ & \\
\hline 1934, July 22 & Hindu Kush.. & $260 \pm 20$ & $250 \pm 20$ & $225 \pm 10$ & $250 \pm 40$ & $210 \pm 35$ & & Small shock \\
\hline 1934, Nov. 18 & Hindu Kush. & $230 \pm 20$ & $220 \pm 10$ & $210 \pm 30$ & $220 \pm 30$ & & & Small shock \\
\hline
\end{tabular}

depth; it is an indication of the scattering of the individual observations, as there is no evidence of systematic error.

The depths found separately for the several Hindu Kush shocks are the same within the limits of error; for all these shocks the focal depth is clearly near 220 $\mathrm{km}$., with a very small error.

In the column headed "Remarks" are given depths determined from other phases, and the values of focal depth found by other investigators.

\section{Preliminary Report on the Shocks Studied}

The following paragraphs contain results on the characteristics of deep-focus earthquakes, obtained incidentally in the study of the selected shocks. This communication is preliminary only; it is expected that the investigation will be continued, and reported on in later publications.

It appears that it will be possible to effect a general improvement in traveltime data by making use of the observations for deep-focus shocks. This is chiefly because these shocks are frequently recorded with very sharp phases, the later of which are not obscured by surface waves (as occurs in normal shocks). 
For preliminary purposes the time residuals of the observed $P$ have been plotted for all the shocks studied. The resulting scatter shows no systematic deviation at any epicentral distance, and consequently leads to no revision of the travel times for $\mathrm{P}$. The corresponding residuals for $\mathrm{S}$ show a more complicated behavior. As mentioned before, there are very many late observations of $\mathrm{S}$ at distances of from $45^{\circ}$ to $55^{\circ}$; it is not yet possible to decide from these data

TABLE 53

Average Differences Observed Minus Calculated Travel Times in Seconds

\begin{tabular}{|c|c|c|c|c|c|c|c|c|}
\hline \multirow{2}{*}{\multicolumn{2}{|c|}{$\begin{array}{l}\text { Date of } \\
\text { shock }\end{array}$}} & \multirow{3}{*}{$\begin{array}{c}\text { Deptb } \\
\text { in km. }\end{array}$} & \multicolumn{5}{|c|}{ Difference observed minus calculated for } & \multirow{2}{*}{ Others } \\
\hline & & & $\mathrm{pP}$ & $\mathrm{pPP}$ & SP & $\mathrm{sP}$ & $\mathrm{BS}$ & \\
\hline 1931, Feb. & 20 & & $1 \pm 3$ & -4 & 1 & 2 & 10 & PPP $0 \pm 8$ \\
\hline 1933, Aug. & 29 & 650 & -3 & & & & & $\mathbf{P}^{\prime} \mathbf{P}^{\prime} 10$ \\
\hline 1932, Jan. & 9 & 380 & $1 \pm 3$ & -10 & & & & \\
\hline 1934, Jan. & 3 & 280 & $0 \pm 4$ & & & $2 \pm 4$ & 10 & $\mathbf{P}^{\prime} \mathbf{P}^{\prime}-9$ \\
\hline 1928, Mar. & 29 & 420 & 1 & & 1 & & 2 & \\
\hline 1933, Sept. & 2 & 410 & $-1 \pm 3$ & -7 & -6 & & & SKPP $P^{\prime}-2$ \\
\hline 1932, April & 4 & 410 & 3 & & & & 0 & \\
\hline 1929, Feb. & 1 & 220 & 3 & -2 & & $2 \pm 2$ & 2 & \\
\hline 1921, Nov. & 15 & 215 & -2 & & & & 2 & Differences for all \\
\hline 1928, Aug. & 10 & 230 & & & & $1 \pm 2$ & 5 & shocks of this group \\
\hline 1931, Oct. & 5 & 220 & $-2 \pm 3$ & 1 & & -1 & 0 & calculated for depth \\
\hline 1933, Jan. & 9 & 220 & $0 \pm 3$ & -6 & & 0 & 0 & of $220 \mathrm{~km}$. \\
\hline 1934, July & 22 & 230 & 1 & 0 & & 4 & 0 & \\
\hline 1934, Nov. & 18 & 220 & $0 \pm 5$ & 5 & & -1 & 6 & \\
\hline
\end{tabular}

whether there is a small $S_{1}$ phase, followed eight or ten seconds later by a large $\mathbf{S}_{2}$, or whether the travel times calculated are actually too early. The same remarks apply to $\mathrm{S}$ in the neighborhood of $70^{\circ}$; here $\mathrm{ScS}$ is frequently reported as $\mathrm{S}$.

Table 53 gives average residuals for various other phases, as determined from the several shocks used. It will be noted that the residuals for $\mathrm{pP}$ and $\mathrm{sP}$ are particularly small. However, sS shows a behavior similar to that of $\mathbf{S}$.

For the selected shocks, the readings of all reported phases have been plotted. All those phases which are consistently reported are found to fall on identifiable travel-time curves; in general the phases thus identified are those which might be expected to be well observed.

The apparent existence of multiplicity in the $S$ phase leads to the question of whether multiplicity is a phenomenon of as general occurrence in deep-focus shocks as it is in normal shocks. ("On Seismic Waves, I," Sect. XXVIII.) To settle this definitely will require study of the data for a large number of deepfocus shocks; however, some tentative conclusions may be drawn from the ap- 
pearance of a limited number of seismograms. Although on the records of longperiod instruments there is frequent indication of multiplicity in $\mathrm{P}$, in the form of clear impulses which are not to be identified with known phases (such as PcP, $\mathrm{pP}$, etc.), the records of short-period instruments show much less motion of this kind. It should be noted that the short-period waves carry a much larger fraction of the energy in deep-focus shocks than they do in normal ones. Earthquakes in which the first $P$ is significantly smaller than the later waves of the multiple group occur rarely if at all. The discussion of possible multiplicity in other phases requires more detailed measurements.

The existence of $\mathrm{pP}, \mathrm{sP}$, etc., has some bearing on the multiplicity found in normal shocks. As has been recognized by Berlage, ${ }^{14}$ these phases should be observable, and must account for part of the observed multiplicity. The causes which produce multiplicity in the direct $\mathrm{P}$ may also act on $\mathrm{pP}$ and $\mathrm{sP}$, and an appreciable number of separable observed waves may arise in this way. However, this type of explanation positively will not account for the very late members of the multiple $P$ group, frequently observed in normal shocks, which often arrive more than a minute after the first $P$. Thus, for a shock at the normal depth of about $25 \mathrm{~km}$., the data used in this paper give $\mathrm{pP}-\mathrm{P}$ as about 8 seconds and $\mathrm{sP}-\mathrm{P}$ about 10 seconds; but these differences must vary greatly with the local crustal structures in the epicentral region.

In "On Seismic Waves, II," Section IV, we have pointed out that deep-focus shocks originating in particular regions regularly record with a uniform type of initial motion (compression from some regions, dilatation from others) at Pasadena; and that the occurrence of prevailing compression or dilatation is the same as for normal shocks in the same region. This relation has persisted for the larger deep-focus shocks recorded since the previous publication, during the additional period January 1, 1935-May 1, 1936. Including these, there are now 19 large deep-focus shocks from South America, all recorded with initial dilatation at Pasadena; 14 shocks from Polynesia (excluding Samoa), 11 of which gave compression; and 6 from the Samoan region, 5 with initial dilatation.

All seven Hindu Kush shocks appear to have been recorded regularly with initial compressions at the European stations, so far as data are available. These seven shocks are a selection from a larger number of shocks having either the same focus or one very close to it. Apparently the repeated occurrence of shocks from the same deep focus is a rather frequent phenomenon. Sometimes the later smaller shocks can hardly be considered otherwise than as aftershocks; but most of the better-established instances refer to the repeated occurrence of strong shocks, often at rather large time intervals.

The geographical distribution of deep-focus shocks remains very definite, and becomes somewhat more so with increasing knowledge, as doubtful occur-

${ }_{14}$ H. P. Berlage, "Sur la profondeur du foyer d'un tremblement de terre déduite d'un seismogramme...," Publ. Bur. Centr. Seism. Inst., (A) 1:5-16 (1924); Handbuch der Geophysik, 4:501 (1932). 
rences are eliminated. Some confusion has resulted from the fact that shocks at slightly greater than normal depth, even as much as $100 \mathrm{~km}$., apparently occur occasionally in almost all the seismic regions of the globe. Shocks at depths of about $200 \mathrm{~km}$. apparently are not so widely distributed as are normal shocks, but they are frequently found in regions where at present there is no evidence of very deep foci. For example, such shocks occur in and around the eastern Mediterranean, in the Hindu Kush, and in the South Atlantic (shock of May 14, 1935).

So far as is now known, shocks originating at the greater depths (400 to 800 $\mathrm{km}$.) are confined to a few regions adjacent to the Pacific basin: Kamchatka and the Kurile Islands, Manchuria, Japan, and southward, the East Indies and Polynesia as far as the Tonga-Kermadec region, and western South America. The South American area appears to be quite isolated from the other regions named, as shocks at really great depths are not known either from North America or from the region of the Antarctic and southeastern Pacific Ocean.

In South America and in the Japanese ${ }^{15}$ and contiguous regions, the epicenters are generally farther removed from the ocean basin the deeper the focus is found to be. In these regions the depth and the distance from the coastal belt of normal shocks are of the same order. Probably a similar relation holds in the Polynesian region, where the limit of normal epicenters, analogous to the bands of normal epicenters along the South American and Japanese coasts, passes north of the main group of Polynesian islands and then turns sharply south through the Tonga and Kermadec deeps. The region of frequent very deep shocks, centering roughly at $25^{\circ} \mathrm{S}, 175^{\circ} \mathrm{E}$, is several hundred kilometers "inland" from this boundary. For the other regions named, especially in the East Indies, correlation between the epicenters of deep and normal shocks is more difficult, as the epicenters of both groups are scattered over a considerable area.

Several authors have given statistical data for the distribution of shock frequency with depth. From their results it is not yet possible to decide whether the number of shocks decreases steadily with increasing depth, or whether there is a maximum at some large depth. It appears not unlikely that particular depths are characteristic of certain areas. It must be borne in mind that the statistics of deep-focus shocks may change with the lapse of time, as new data accumulate. The deepest foci found so far are at depths of about $700 \mathrm{~km}$; ; yet some of the shocks at these extreme depths are among the very largest recorded.

The problem of the causative mechanism of these shocks has not yet been solved completely. The complex patterns of initial observed compressions and dilatations, and the frequent observation of large shear waves (S), make explanations in terms of an explosive or collapse type of origin improbable; apparently the originating movement is accompanied by shear. The geographical distribution of shocks recorded with initial compression or dilatation at

${ }^{15} \mathrm{~K}$. Wadati, "On the Activity of Deep-Focus Earthquakes in the Japan Islands and Neighbourhoods," The Geophysical Magazine, 8:305-326 (1935). 
Pasadena indicates that in general this shearing or faulting movement has the same direction over large areas, persists over long periods of time, and is frequently the same for all focal depths in the given region (including normal shocks).

The fact that large stresses can accumulate at great depths does not necessarily imply great strength at these depths; for, as has been shown by Haskell, ${ }^{16}$ the high coefficient of viscosity prevents rapid flow even if there is no strength. As is shown by the recurrence of shocks from the same focus, only a few years may be required for the accumulation of stresses sufficient to produce a large earthquake. On the other hand, during such a time interval, theoretically only a small fraction of the stress should be removed by plastic flow. The calculations of Haskell show further that the stresses at the surface are accompanied by only slightly smaller stresses at depths of a few hundred kilometers, and vice versa. This would explain the occurrence of shallow and deep earthquakes in the same regions. The results found by Stetson ${ }^{17}$ on the periodic relation of deep-focus earthquakes to the tidal forces might also be explained in this way.

The most probable conclusion, in the present state of our knowledge, is that normal and deep-focus earthquakes are brought about by the same forces, which may act either near the surface or at great depths.

\section{Summary (Abstract)}

Travel times of normal earthquakes are made the basis for calculated travel times for shocks at depths down to $800 \mathrm{~km}$. These are presented in tabular form, together with certain auxiliary data. Theoretical discussion is given for the critical distances at which $\mathrm{pP}$ and $\mathrm{PP}, \mathrm{sP}$ and $\mathrm{SP}$, etc., coincide, and below which these phases should not occur. These distances are not focal points (caustics). Methods are given for determining epicenter, depth, and origin time, and are applied to a selected group of shocks. The results of different methods agree very well with one another and with the calculated travel-time data. A preliminary report is given on certain characteristics of the shocks studied. The mechanism of deep-focus earthquakes is discussed briefly, and it is concluded that normal and deep-focus earthquakes are probably brought about by the same forces.

\footnotetext{
${ }^{16} \mathrm{H}$. Haskell, "The Motion of a Viscous Fluid Under a Surface Load," Physics, 6:265-269 (1935), and 7:56-61 (1936).

${ }^{17}$ H. T. Stetson, "The Correlation of Deep-Focus Earthquakes with Lunar Hour Angle and Declination," Science, 82:523-524 (1935).
}

Balch Graduate School of the Geological Sctences,

California Institute of Technology, [Contribution No. 207]

Pasadena, California.

Carnegie Institution of Washington, Seismological Research, Pasadena, California. 


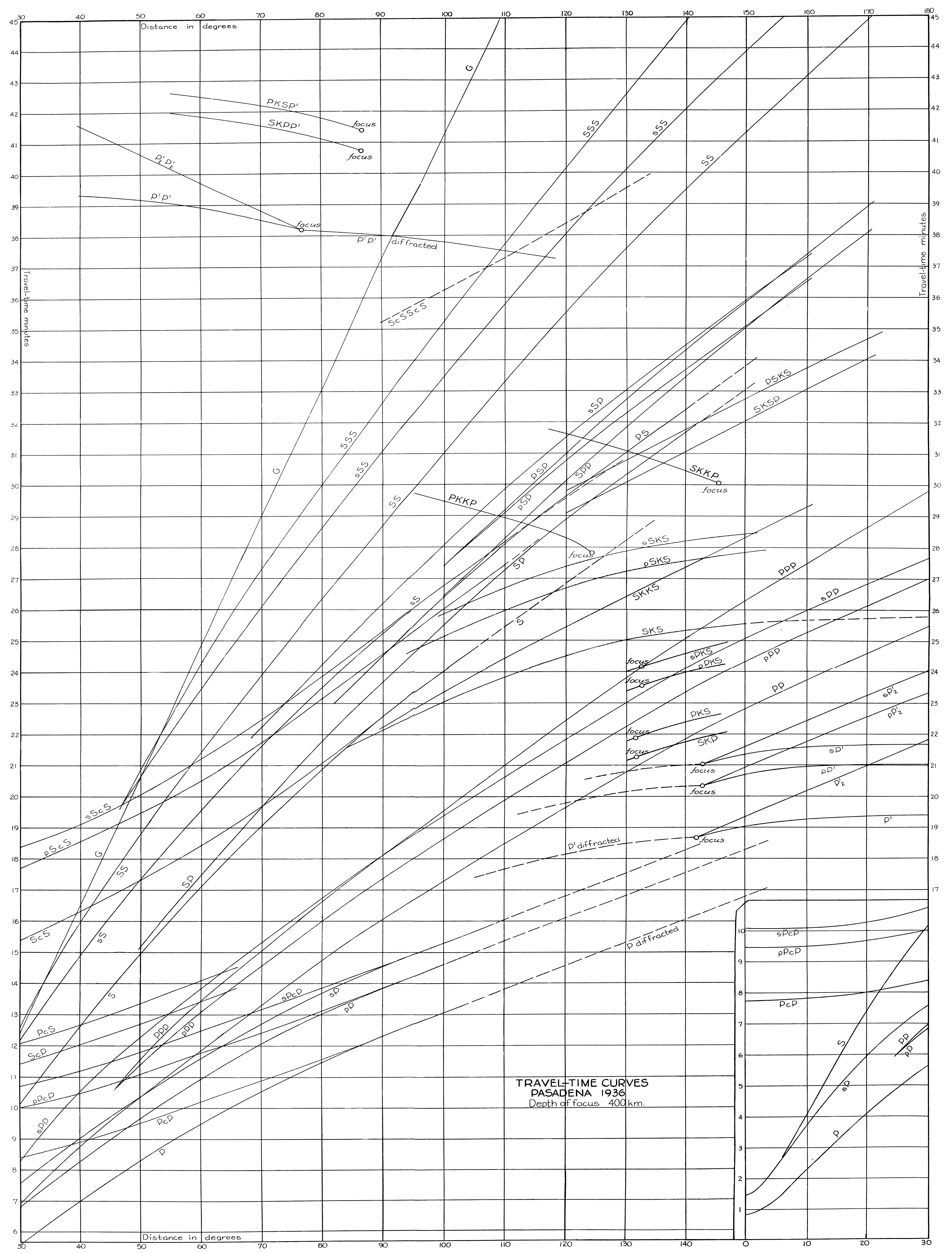

